\title{
Integrated Science Investigation of the Sun (ISIS): Design of the Energetic Particle Investigation
}

\author{
D.J. McComas · N. Alexander • N. Angold · S. Bale • C. Beebe $\cdot$ B. Birdwell •
}

M. Boyle · J.M. Burgum • J.A. Burnham - E.R. Christian · W.R. Cook · S.A. Cooper •

A.C. Cummings • A.J. Davis • M.I. Desai - J. Dickinson • G. Dirks • D.H. Do • N. Fox •

J. Giacalone · R.E. Gold • R.S. Gurnee · J.R. Hayes • M.E. Hill • J.C. Kasper •

B. Kecman • J. Klemic • S.M. Krimigis • A.W. Labrador • R.S. Layman • R.A. Leske •

S. Livi - W.H. Matthaeus · R.L. McNutt Jr • R.A. Mewaldt • D.G. Mitchell •

K.S. Nelson - C. Parker · J.S. Rankin • E.C. Roelof • N.A. Schwadron · H. Seifert •

S. Shuman • M.R. Stokes · E.C. Stone • J.D. Vandegriff • M. Velli •

T.T. von Rosenvinge $\cdot$ S.E. Weidner $\cdot$ M.E. Wiedenbeck $\cdot$ P. Wilson IV

Received: 21 February 2014 / Accepted: 16 June 2014 / Published online: 5 July 2014

(C) The Author(s) 2014. This article is published with open access at Springerlink.com

\begin{abstract}
The Integrated Science Investigation of the Sun (ISIS) is a complete science investigation on the Solar Probe Plus (SPP) mission, which flies to within nine solar radii of the Sun's surface. ISIS comprises a two-instrument suite to measure energetic parti-
\end{abstract}

D.J. McComas $(\bowtie) \cdot$ N. Alexander · N. Angold · C. Beebe · B. Birdwell · M.I. Desai · J. Dickinson · G. Dirks · S. Livi · S.E. Weidner · P. Wilson IV

Southwest Research Institute, San Antonio, TX 78228, USA

e-mail: dmccomas@swri.edu

D.J. McComas · M.I. Desai · S. Livi

University of Texas at San Antonio, San Antonio, TX 78249, USA

S. Bale

University of California at Berkeley, Berkeley, CA 94720, USA

M. Boyle · J.M. Burgum - S.A. Cooper · D.H. Do · N. Fox · R.E. Gold · R.S. Gurnee · J.R. Hayes · M.E. Hill · S.M. Krimigis · R.S. Layman · R.L. McNutt Jr · D.G. Mitchell · K.S. Nelson · C. Parker · E.C. Roelof · H. Seifert · M.R. Stokes · J.D. Vandegriff

Johns Hopkins University Applied Physics Laboratory, Laurel, MD 20723, USA

J.A. Burnham - W.R. Cook · A.C. Cummings · A.J. Davis · B. Kecman · J. Klemic · A.W. Labrador ·

R.A. Leske - R.A. Mewaldt - J.S. Rankin - E.C. Stone

California Institute of Technology, Pasadena, CA 91125, USA

E.R. Christian · S. Shuman · T.T. von Rosenvinge

Goddard Space Flight Center, Greenbelt, MD 20771, USA

J. Giacalone

University of Arizona, Tucson, AZ 85721, USA

J.C. Kasper

University of Michigan, Ann Arbor, MI 48109, USA 
cles over a very broad energy range, as well as coordinated management, science operations, data processing, and scientific analysis. Together, ISIS observations allow us to explore the mechanisms of energetic particles dynamics, including their: (1) Originsdefining the seed populations and physical conditions necessary for energetic particle acceleration; (2) Acceleration-determining the roles of shocks, reconnection, waves, and turbulence in accelerating energetic particles; and (3) Transport-revealing how energetic particles propagate from the corona out into the heliosphere. The two ISIS Energetic Particle Instruments measure lower (EPI-Lo) and higher (EPI-Hi) energy particles. EPI-Lo measures ions and ion composition from $\sim 20 \mathrm{keV} /$ nucleon-15 MeV total energy and electrons from $\sim 25-1000 \mathrm{keV}$. EPI-Hi measures ions from $\sim 1-200 \mathrm{MeV} /$ nucleon and electrons from $\sim 0.5-6 \mathrm{MeV}$. EPI-Lo comprises 80 tiny apertures with fields-of-view (FOVs) that sample over nearly a complete hemisphere, while EPI-Hi combines three telescopes that together provide five large-FOV apertures. ISIS observes continuously inside of $0.25 \mathrm{AU}$ with a high data collection rate and burst data (EPI-Lo) coordinated with the rest of the SPP payload; outside of $0.25 \mathrm{AU}$, ISIS runs in low-rate science mode whenever feasible to capture as complete a record as possible of the solar energetic particle environment and provide calibration and continuity for measurements closer in to the Sun. The ISIS Science Operations Center plans and executes commanding, receives and analyzes all ISIS data, and coordinates science observations and analyses with the rest of the SPP science investigations. Together, ISIS' unique observations on SPP will enable the discovery, untangling, and understanding of the important physical processes that govern energetic particles in the innermost regions of our heliosphere, for the first time. This paper summarizes the ISIS investigation at the time of the SPP mission Preliminary Design Review in January 2014.

Keywords Solar Probe Plus · ISIS · Solar energetic particles · SEPs · CMEs · Corona · Particle acceleration

\section{Introduction}

\subsection{Science Background and Objectives}

NASA's Solar Probe Plus (SPP) mission is designed to plunge repeatedly into the innermost regions of the solar system where the Sun's million-degree atmosphere, or corona, begins its outward expansion to produce the supersonic solar wind. The outward-flowing solar wind interacts with the Earth's magnetosphere and other objects in the solar system and ultimately inflates a bubble in the interstellar medium known as the heliosphere, which engulfs and protects the Earth and the planets from galactic cosmic radiation. SPP is designed to survey the

J.C. Kasper

Harvard-Smithsonian Center for Astrophysics, Cambridge, MA 02138, USA

W.H. Matthaeus

University of Delaware, Newark, DE 19716, USA

N.A. Schwadron

University of New Hampshire, Durham, NH 03824, USA

M. Velli · M.E. Wiedenbeck

Jet Propulsion Laboratory, California Institute of Technology, Pasadena, CA 91109, USA 
birthplace of the solar wind and its embedded magnetic and electric fields, explore the origins of large-scale disturbances created during powerful explosions known as solar flares and coronal mass ejections (CMEs), and reveal how these conspire to accelerate solar energetic particles (SEPs) — suprathermal and energetic particles from $\sim$ few $\mathrm{keV}$ up to $\mathrm{GeV}$. By making direct in-situ measurements of the inner heliospheric environment from $<10$ to $>60$ solar radii $\left(R_{S}\right)$, SPP is poised to redefine Solar and Heliospheric physics as we currently understand them (see McComas et al. 2007).

Solar Probe Plus (Fox et al. 2014) carries a complement of four state-of-the-art scientific instruments/instrument suites: a solar wind plasma suite- the Solar Wind Electrons Alphas and Protons (SWEAP) investigation (Kasper et al. 2014); an electric and magnetic field suite- "FIELDS" (Bale et al. 2014); a wide field imager- "WISPR" (Howard et al. 2014); and the energetic particle suite - the Integrated Science Investigation of the Sun (ISIS). ISIS is designed to provide comprehensive measurements of the energetic particle environment, including energy spectra, anisotropy, and composition of suprathermal and solar energetic ions from $\sim 0.02-200 \mathrm{MeV} /$ nucleon (nuc), as well as the energy spectra and arrival direction of $\sim 0.025-6 \mathrm{MeV}$ electrons. Within ISIS, observations are made with two complementary instruments: Energetic Particle Instrument-Low energy (EPI-Lo) and Energetic Particle Instrument-High energy (EPI-Hi), which measure the lower and higher energy energetic particles, respectively. This paper describes the science goals and instrumentation of the ISIS investigation as planned and designed as of SPP's Preliminary Design Review (PDR) in January 2014.

The overall science objective of ISIS is to explore the physical mechanisms that produce, accelerate, and transport energetic particles in the inner heliosphere. ISIS achieves this objective by addressing the following three questions:

1. What is the origin or seed population of solar energetic particles (SEPs)?

2. How are these SEPs and other particle populations accelerated?

3. What mechanisms are responsible for transporting the different particle populations into the heliosphere?

ISIS additionally contributes to addressing SPP's other two overarching scientific objectives, namely, (1) Trace the flow of energy that heats and accelerates the solar corona and solar wind and (2) Determine the structure and dynamics of the plasma and magnetic fields at the sources of the solar wind through its measurements of suprathermal particle ( 2-400 keV/nuc H-Fe ions) tails. The ISIS design is also set to reveal previously unknown features of the inner heliosphere, which stretches from the Sun's corona to the orbit of Mercury. Thus, we also anticipate great discovery science from ISIS and more generally from the SPP mission.

The ISIS investigation is carried out by a world-class team of scientists, engineers, technicians, and business and support personnel; Fig. 1 provides ISIS' organizational chart and identifies the key team members leading in each area and Table 1 lists the Science Team members. The team was assembled by combining top people and institutions that have been highly successful across a broad range of prior missions. ISIS leadership works to foster an environment where everyone works together to produce even greater synergies and uses the combined experience and capabilities of the team to make ISIS the best possible science investigation for SPP.

The remainder of Sect. 1 provides a scientific justification for suprathermal and energetic particle measurements on SPP and discusses each of the above science questions in terms of the several particle populations that SPP will encounter. The focus is on particular topics that only the ISIS investigation can address. Section 2 provides an overview of ISIS, 


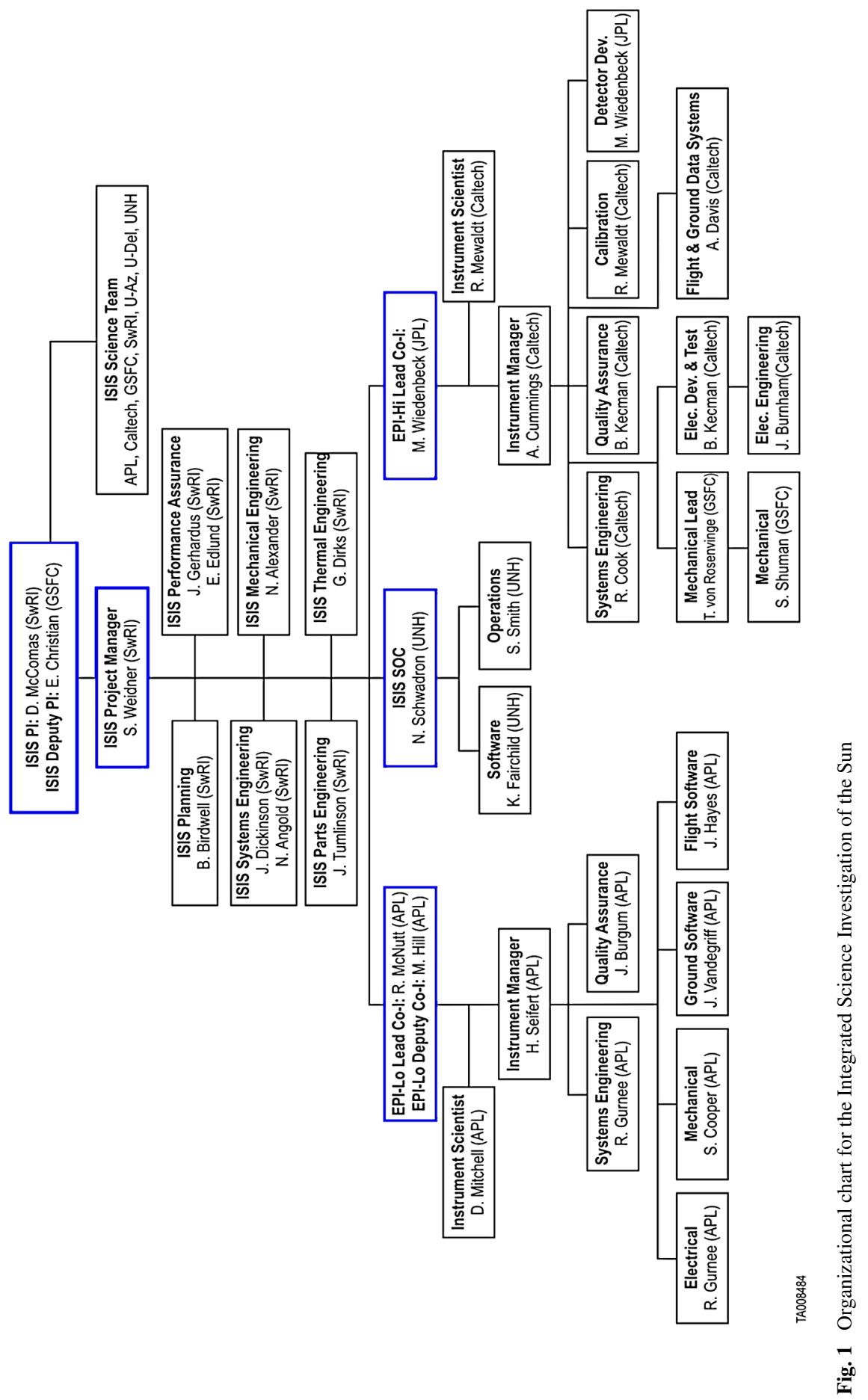


Table 1 ISIS Science Team members

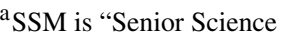

Mentor": Non-Co-I science team members who provide senior-level guidance and advice to the rest of the team

\begin{tabular}{lll}
\hline Title & Name & Organization \\
\hline PI & Dave McComas & SwRI \\
DPI & Eric Christian & GSFC \\
Co-I & Alan Cummings & Caltech \\
Co-I & Mihir Desai & SwRI \\
Co-I & Joe Giacalone & U Arizona \\
Co-I & Matthew Hill & JHU/APL \\
Co-I & Stefano Livi & SwRI \\
Co-I & Bill Matthaeus & U Delaware \\
Co-I & Ralph McNutt & JHU/APL \\
Co-I & Dick Mewaldt & Caltech \\
Co-I & Don Mitchell & JHU/APL \\
Co-I & Nathan Schwadron & UNH \\
Co-I & Tycho von Rosenvinge & GSFC \\
Co-I & Mark Wiedenbeck & JPL \\
SSM & Robert Gold & JHU/APL \\
SSM & Stamatios Krimigis & JHU/APL \\
SSM & Edmond Roelof & JHU/APL \\
SSM $^{\mathrm{a}}$ & Ed Stone & Caltech \\
\hline
\end{tabular}

including suite philosophy, the ISIS suite viewing, mechanical and electrical aspects of the overall design, and plans for science data and operations. Sections 3 and 4 provide details of the EPI-Lo and EPI-Hi instruments, respectively, including overall design, planned and expected performance vs. requirements, electrical and mechanical design, and calibration plans; Sect. 5 describes planned science operations, data processing, and data products in more detail, while Sect. 6 provides a brief summary.

\subsection{Solar Energetic Particles, Solar Flares, and Coronal Mass Ejections}

\subsubsection{Two Sources of Solar Energetic Particles}

Prior to the mid-1980s, solar flares were believed to produce much of the solar energetic particle population observed near Earth orbit. However, this viewpoint was challenged when coronal mass ejections (CMEs) were first observed and then recognized as powerful alternative means by which the Sun expels considerable matter and energy into the heliosphere and causes geomagnetic disturbances (e.g., Gosling 1993, and references therein). As illustrated in Fig. 2, it is now widely accepted that the Sun accelerates charged particles (ions and electrons) from $\sim$ few $\mathrm{keV}$ up to near-relativistic energies in at least two ways (e.g., Reames 2013). Mechanisms associated with magnetic reconnection occur during solar flares and are largely responsible for producing the smaller "impulsive" SEP events that are enriched in electrons, ${ }^{3} \mathrm{He}$, and heavier ions such as Ne-Fe (e.g., Mason 2007). In contrast, large-scale shock waves and related processes, driven by fast CMEs plowing through the ambient corona and the solar wind are associated with the larger "gradual" SEP events (Reames 1999). Though the acceleration mechanisms in the two cases are distinct, and both are highly efficient, their effects are often intertwined. The net result is that charged particles are frequently energized up to $\mathrm{GeV}$ energies within a few seconds to minutes (Rice et al. 2003). 

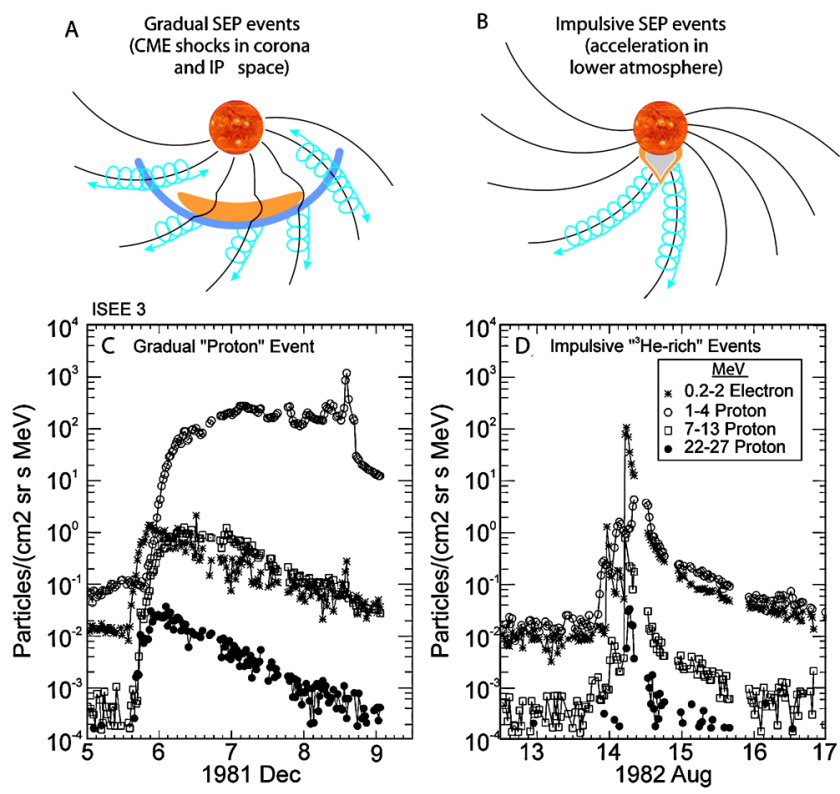

Fig. 2 Two sources of SEP events. (A) A large gradual event is produced by an expanding CME-driven shock wave that populates interplanetary magnetic field (IMF) lines with SEPs over a broad longitudinal extent. (B) A solar flare produces an impulsive event that populates well-connected IMF lines, leaving nearby IMF lines relatively empty. Intensity-time profiles of electrons and protons in $(\mathbf{C})$ a large gradual and (D) a small impulsive SEP event (adapted from Reames 1999)

Fig. 3 Electron

$(e: 0.3-0.8 \mathrm{MeV})$ and $\mathrm{He}$ ( $\alpha: 2-4 \mathrm{MeV} /$ nuc) time profiles from Helios-1 (0.3 AU) and IMP-8 (1 AU) during five impulsive SEP events in 1980 (from Wibberenz and Cane 2006). Magnetic connections to the flare site are indicated at upper right. Helios-1 observed five injections that had merged into a single event by the time they reached IMP-8

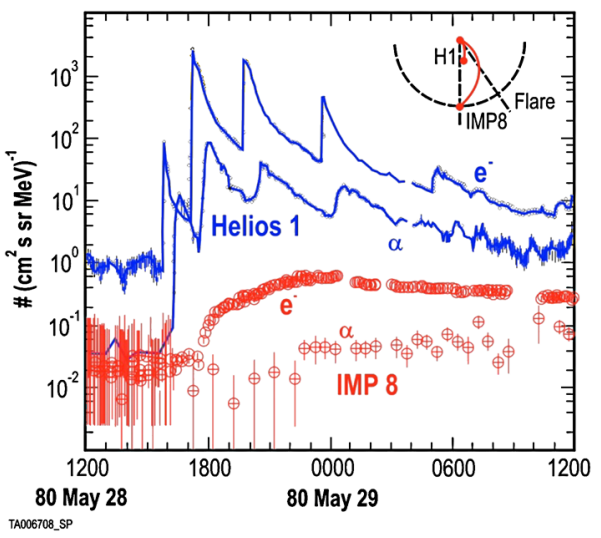

\subsubsection{Why Do We Need to Make Measurements Closer to the Sun?}

An impediment to understanding the origin and acceleration mechanisms and developing and testing physics-based models using near-Earth observations is that by the time SEPs reach 1 AU, particles from multiple sources (flares, CME shocks etc.), having been accelerated by different physical processes (e.g., reconnection, shock acceleration), are completely mixed. Figure 3 shows a clear example of the need for SEP observations in the inner heliosphere. For these observations, the Helios- 1 spacecraft located at $\sim 0.4$ AU detected at least 


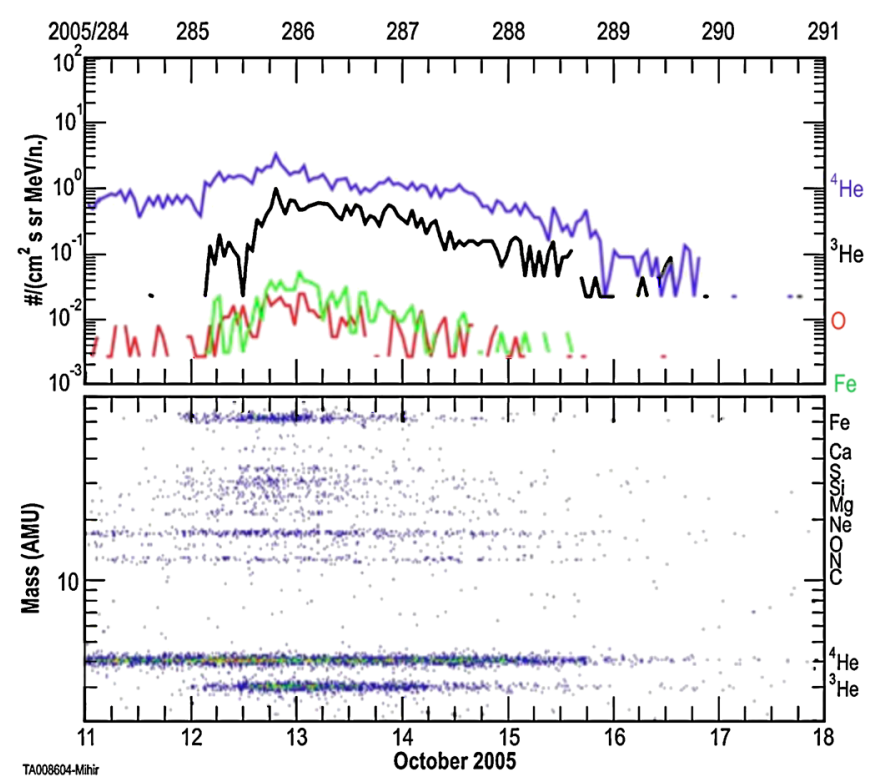

Fig. 4 Top: Time-intensity profiles of ${ }^{3} \mathrm{He},{ }^{4} \mathrm{He}, \mathrm{Fe}$, and $\mathrm{O}$ and mass spectrogram of ions with mass between 3 and 60 AMU during a 7-day period (taken from Mason 2007)

5 separate impulsive-like injections of electrons and He ions, while IMP-8 at 1 AU observed a single particle event. The dramatically different time-intensity profiles could result from a combination of one or more of the following processes: (1) scattering and diffusion during transport between 0.4 and $1 \mathrm{AU}$; (2) distinct magnetic connections of the spacecraft to the particle sources; and (3) the spatio-temporal evolution of the particle sources. These mechanisms smear the time profiles at $1 \mathrm{AU}$, making it difficult to identify the source locations and therefore understand the nature of the acceleration mechanisms. By making SEP measurements close to and, in some cases, within the acceleration region and at various radial distances along the way, ISIS allows us to understand and untangle the effects of transport, including velocity dispersion, for the first time.

\subsection{Identify the Origins of Energetic Particles in the Inner Heliosphere}

\subsubsection{Impulsive SEP Events}

Combined observations of the energy spectra and onset times of ${ }^{3} \mathrm{He}$-rich impulsive SEP events and $>5 \mathrm{keV}$ electrons with imaging of Type III radio bursts and Extreme ultraviolet (EUV) observations of the flaring regions indicate that many impulsive SEP events are associated with jet-like ejections that occur around the solar particle release times (Pick et al. 2006). From extrapolations using Potential Field Source Surface models, as in Wang et al. (2006), the source regions of these particles are seen to be adjacent to coronal holes containing Earth-directed open field lines. In this scenario, the jets are signatures of magnetic reconnection or exchange of connectivity between closed field lines in the source regions and Earth-directed open field lines, and the discrete ${ }^{3} \mathrm{He}$-rich particle events seen in Fig. 4 are observed when such foot point exchanges occur. While this model could account for two discrete ${ }^{3} \mathrm{He}$-rich increases seen in the time-intensity profiles in Fig. 4 on October 12, 2005, 
it is unclear how it could also explain the presence of ${ }^{3} \mathrm{He}$ for a period of more than $\sim 3$ days thereafter. Mason (2007) suggested that a nearly continuous emission process associated with magnetic reconnection between closed and open field lines may be associated with the acceleration and subsequent release of ${ }^{3} \mathrm{He}$ into the interplanetary medium. In addition, the three physical mechanisms discussed in Sect. 1.2.2 could also play important roles in producing long duration ${ }^{3} \mathrm{He}$-rich intervals. Because the expected intensities and therefore the effective sensitivity will be significantly higher in the inner heliosphere, SPP will measure discrete injections of ${ }^{3} \mathrm{He}$ during numerous SEP events that are smaller than currently detected from Earth-orbit. In addition, by combining observations of energetic electrons, Type-III radio bursts and other imaging observations, SPP will also be able to identify and associate periods of continuous ${ }^{3} \mathrm{He}$ emission with nearly steady coronal processes involving foot point exchange and magnetic reconnection.

These ISIS measurements, in conjunction with FIELDS and SWEAP, will also contribute to another SPP scientific objective, to trace the flow of energy that heats and accelerates the solar corona and solar wind. In particular ISIS, FIELDS and SWEAP enable studies to distinguish among heating mechanisms, such as reconnection, ion-cyclotron waves, and turbulent dissipation and associated stochastic heating (Cranmer and van Ballegooijen 2010; Isenberg and Vasquez 2013; Cranmer et al. 2007; Chandran et al. 2013). Direct parallel acceleration of suprathermals by reconnection up to several times the Alfvén speed in the low beta corona may be diagnosed by ISIS measurement of pitch angle distributions and energy spectra of electrons and energetic ions. Stochastic acceleration associated with Fermi processes and propagating waves, a process familiar in flares and astrophysical applications (Liu et al. 2004), will produce bidirectional beaming distributions of energized ions and widely varying energy spectra features. In contrast, stochastic acceleration of the betatron type may occur near (but outside of) reconnecting current sheets (Dmitruk et al. 2004; Dalena et al. 2014; Teaca et al. 2014) and may produce higher energy suprathermals with perpendicular pitch angle distributions. Finally, ion cyclotron resonance can produce distinctive pitch angle signatures associated with a population of resonant waves of the required polarization (Isenberg and Vasquez 2013). ISIS measurement of pitch angle anisotropies and energy spectra together with FIELDS magnetic field and electric field spectral energy density, polarization and helicities, and SWEAP ion velocities and temperatures, will allow direct and correlative measurements that will distinguish among these mechanisms. These studies will be done at various heliocentric distances approaching and within the Alfvén radius, enabling an evaluation of the radial variation of the relative effects of these distributed heating mechanisms.

\subsubsection{Large Gradual SEP Events}

As the outward moving CMEs expand and slow down, the associated shocks weaken, implying that most CME shocks are very efficient at accelerating particles when they are close to the Sun and produce most of the higher energy ions within $\sim 20 \mathrm{R}_{\mathrm{S}}$. Since CMEs propagate into the ambient corona and the slow solar wind, the associated shocks were generally believed to inject and accelerate coronal or solar wind material (e.g., Reames 1999). However, the ion composition in many large gradual CME-associated SEP events was enriched in the rare isotope ${ }^{3} \mathrm{He}$ and in heavy ions such as $\mathrm{Ne}-\mathrm{Fe}$, leading some researchers (see e.g., Mason et al. 1999) to suggest that CME shocks encounter and re-accelerate suprathermal flare material that is frequently present in the interplanetary medium (Wiedenbeck et al. 2008). In contrast, Cane et al. (2006) suggested that flares make a direct contribution to many large gradual SEPs, especially at energies above $\sim 10 \mathrm{MeV} /$ nuc. By making the first-ever in-situ 

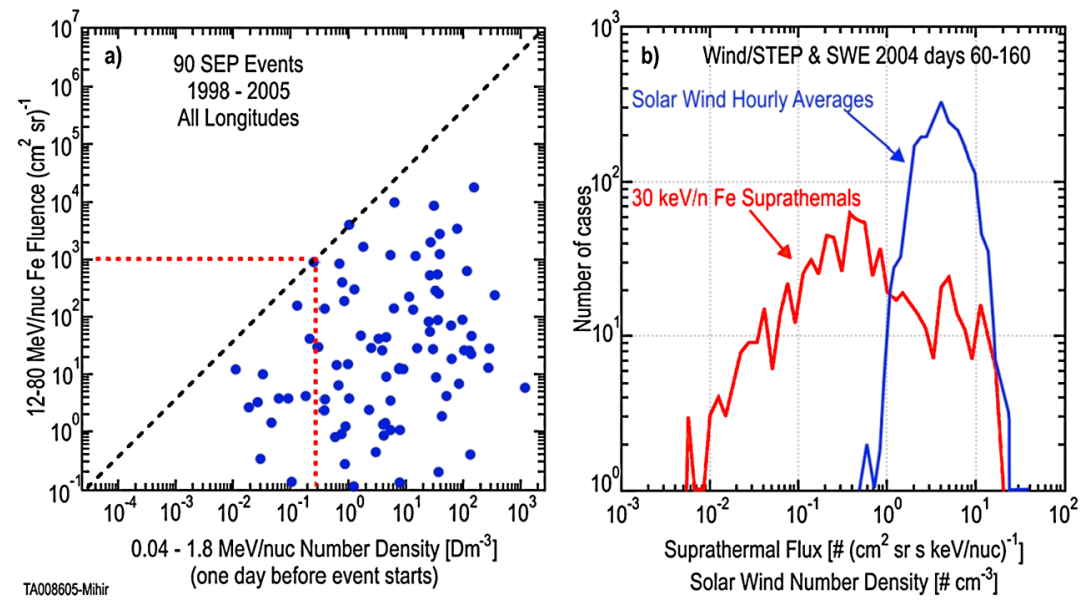

Fig. 5 Left: Fluence of $>12 \mathrm{MeV} /$ nuc Fe in large SEP events vs. suprathermal Fe density one day prior to the onset of the corresponding SEP event (after Mewaldt et al. 2012b). Right: Hourly averaged intensity of suprathermal $\sim 30 \mathrm{keV} / \mathrm{nuc} \mathrm{Fe}$ (red) and number density of solar wind Fe (blue) for a 100 day period in 2004 (taken from Mason et al. 2005)

measurements of the properties of CME shocks, suprathermal ions, and the accelerated SEPs simultaneously within $20 \mathrm{R}_{\mathrm{S}}$ combined SPP measurements will help reveal how flares contribute to large gradual SEPs, either by providing the suprathermal seed population or by providing the higher energy SEPs themselves. A key element in unraveling the balance of these effects will be ISIS analyses of transport effects, discussed further below.

\subsubsection{Suprathermal Ions}

The presence of rare $\left(\sim 10^{-4}\right.$ of $\left.{ }^{4} \mathrm{He}\right)$ solar wind ion species such as ${ }^{3} \mathrm{He}$ and $\mathrm{He}^{+}$in substantial amounts in the gradual SEP population indicates that the associated CME-driven shocks draw much of their seed population from a pool of suprathermal ions with speeds above that of the ambient solar wind ions (e.g., Desai et al. 2001), at least for these species, and possibly others as well. Ions from multiple sources can contribute to a commonly observed suprathermal tail ( $\sim 1.5-20$ times the solar wind speed). Suprathermal ions are defined here as $\sim 2-400 \mathrm{keV} /$ nuc $\mathrm{H}-\mathrm{Fe}$ ions. These sources can be highly variable in space and time, and include gradual and impulsive SEP events, CME-driven interplanetary shocks, and corotating interaction regions (CIRs). Seed particles may also be extracted from the pool of heated and accelerated solar wind ions, and interstellar and inner source pickup ions.

Figure 5 demonstrates this connection to more energetic particles that provides an important motivation to understand the physical mechanisms responsible for the production of suprathermal (ST) ions. The left panel shows that the $>12 \mathrm{MeV} /$ nuc Fe fluence near Earth in large SEP events is likely to be significantly larger if the ST ion intensities $\sim 1$ day prior to the occurrence of the corresponding SEP event were also high (Mewaldt et al. 2012b). The right panel shows that the $>30 \mathrm{keV} /$ nuc ST Fe intensities vary by three orders in magnitude as compared with the factor of $\sim 10$ variations in the solar wind densities (Mason et al. 2005). Such variations in the ST ion populations are expected to play a critical role in determining what types of seed populations are available for acceleration by CME shocks.

Existing observations of ST ion populations, as shown in Figs. 6 and 7, tell somewhat conflicting stories (Mason and Gloeckler 2012). For instance, Fig. 6 shows that the ST pro- 




Fig. 6 Proton phase space densities exhibit a common spectra shape under a variety of solar wind conditions (taken from Fisk and Gloeckler 2012)

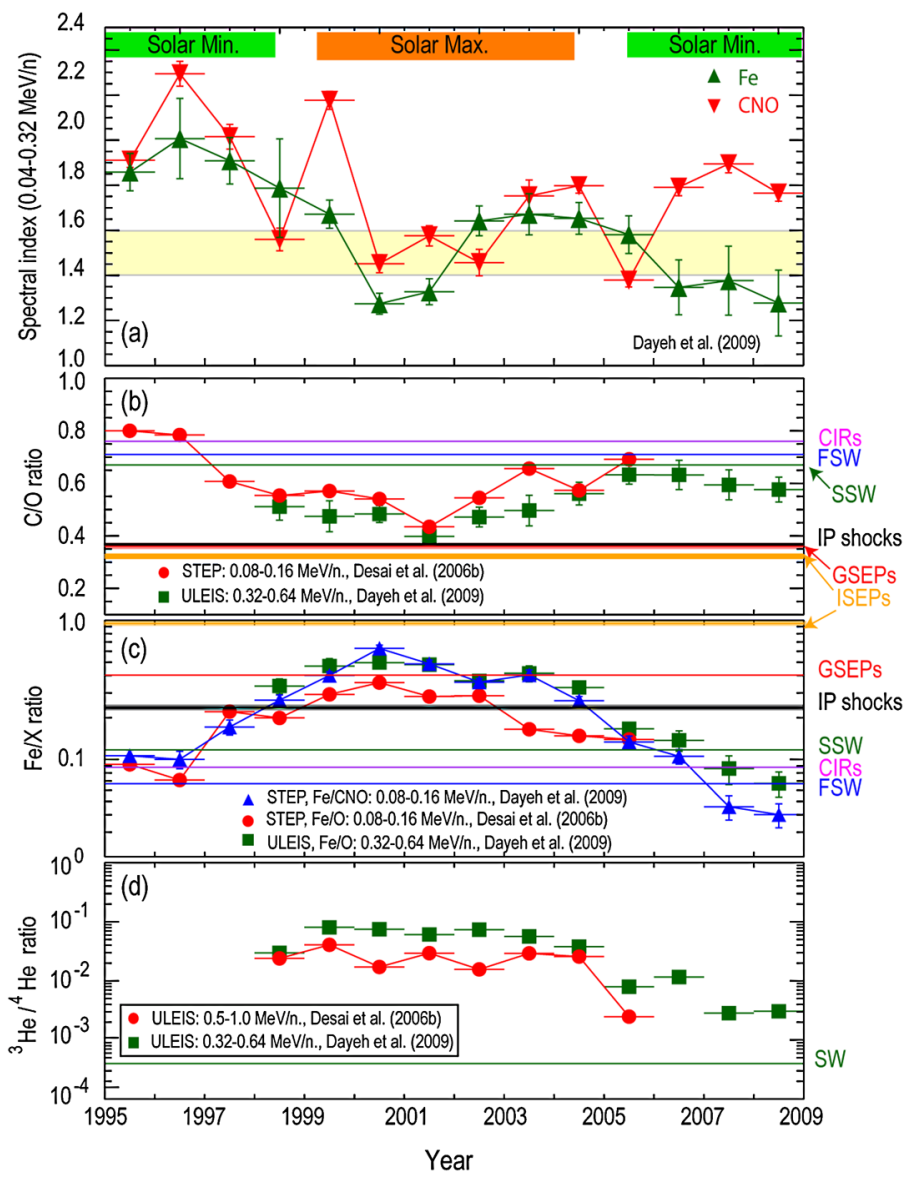

Fig. 7 Annual averages of suprathermal Fe and CNO spectral indices, $\mathrm{C} / \mathrm{O}$ ratio, Fe/CNO ratio, and ${ }^{3} \mathrm{He} /{ }^{4} \mathrm{He}$ ratio (adapted from Desai et al. 2006; Dayeh et al. 2009) 
ton tails between $\sim 1.5-6$ times the solar wind speed are characterized by an energy powerlaw with a nearly constant spectral index of -1.5 (i.e., $E^{-1.5}$, corresponding to a distribution function $f(v) \sim v^{-5}$, where $v$ is particle velocity) under a variety of solar wind conditions, with most enhancements in the ST tail density being associated with extended compression regions (Fisk and Gloeckler 2012). In contrast, Fig. 7 shows that the ST tail between 6-20 times the solar wind speed has highly variable spectral indices and that the ion composition varies with solar activity cycle, implying that the contributions from various sources (e.g., CIRs, SEPs etc.) also vary in time and space (Dayeh et al. 2009). Giacalone (2012) showed that strong interplanetary shocks are significant sources of suprathermal ions and that the peak enhancement of the particles occurred at the same time as the passage of nearly all the shocks studied. This suggests that shocks may also be an important a source for the ever-present, but variable, suprathermal particle tail given that energetic particles, being quite mobile, can remain within the inner heliosphere for some time.

Present theoretical models regarding the origin and acceleration of suprathermal ions can be grouped into two categories: (1) ST tails result from a distributed, continuous acceleration process. In interplanetary space this could be any second order Fermi process with (the problematic) constraint that acceleration and escape times are essentially equal. Specific suggestions have been made to achieve this, including distributed reconnection embedded in solar wind turbulence (Le Roux et al. 2002) and acceleration in extended compression regions (Fisk and Gloeckler 2012). SPP also could discover that ST tails originate in the solar corona due to micro- or nano-flares, a possibility that once again would have implications for observed signatures of transport; or (2) ST tails are lower energy portions of material accelerated in energetic particle events such as CIRs, transient interplanetary shocks (Giacalone 2012), SEPs etc. (Livadiotis and McComas 2009; Jokipii and Lee 2010; Schwadron et al. 2010).

Adding to these controversies is the fact that, under typical solar wind conditions, current observations either do not have the capability, or lack the sensitivity, to measure the composition of suprathermal ions below $\sim 50 \mathrm{keV} /$ nuc to discern contributions from various sources on shorter timescales (less than a day). If the suprathermal ion tails are produced by CME shocks and solar flare-reconnection driven processes (e.g., nano flares), then the tail fluxes are likely to be significantly higher in the inner heliosphere; therefore ISIS observations that can simultaneously measure both suprathermal protons and heavy ions up to $\mathrm{Fe}$ will determine how the contributions from different sources vary on shorter timescales and with distance.

\subsection{Understand SEP Acceleration Mechanisms}

\subsubsection{Impulsive SEP Events}

Magnetic reconnection-associated mechanisms during powerful solar flares accelerate electrons and ions to near-relativistic energies; these may involve first order Fermi processes as well as second order (stochastic) acceleration, or a mixture of first and second order processes (Ambrosiano et al. 1988). Acceleration via reconnection can be complex, involving direct acceleration, trapping particles in collapsing magnetic islands (Drake et al. 2006), stochastic acceleration (Le Roux et al. 2002; Chandran et al. 2010), and perpendicular betatron acceleration (Dmitruk et al. 2004; Dalena et al. 2014). Stochastic and reconnection-related acceleration mechanisms may be driven by low frequency fluctuations associated with MHD turbulence, or by plasma waves that may preferentially heat the ${ }^{3} \mathrm{He}$ nuclei and heavy ions, while also preferentially occurring near disturbances such 

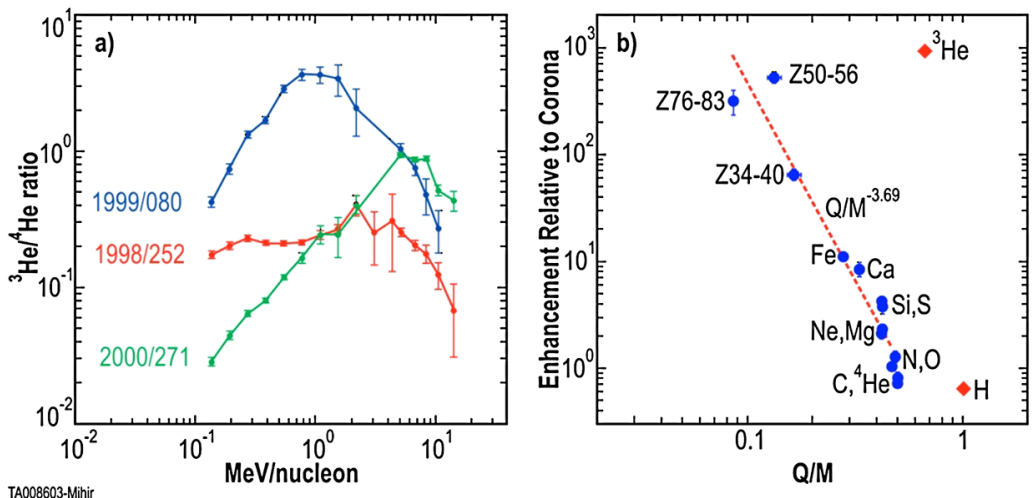

Fig. 8 Left: Energy dependence of the ${ }^{3} \mathrm{He} /{ }^{4} \mathrm{He}$ ratio in 3 individual ${ }^{3} \mathrm{He}$-rich impulsive SEPs. Right: Average abundance enhancement factor in impulsive SEP events vs. Q/M, assuming that the ionic charge states reflect an equilibrium plasma temperature of 3.2 MK. The power-law dependence (red line) has a slope of -3.26 (from Mason 2007)

as shocks. Various theoretical models (e.g., Aschwanden 2002; Petrosian et al. 2009; Kartavykh et al. 2008) are partially successful in accounting for some observed features in impulsive SEP events, but none has so far explained all of the key observations simultaneously. These observed features include:

1. ${ }^{3} \mathrm{He} /{ }^{4} \mathrm{He}$ abundance ratios significantly enhanced relative to the ambient corona (Mason 2007).

2. Event-to-event variability in spectral forms; spectra for all species are either power-laws or broken power-laws with nearly constant abundance ratios $\left(\mathrm{Fe} / \mathrm{O}\right.$ and $\left.{ }^{3} \mathrm{He} /{ }^{4} \mathrm{He}\right)$ with energy or they exhibit curvature for only ${ }^{3} \mathrm{He}$ and $\mathrm{Fe}$, leading to dramatic energy-dependent abundances (left panel in Fig. 8, taken from Mason et al. 2002).

3. Power-law behavior of the $\mathrm{Q} / \mathrm{M}$-dependent enhancements in the abundances of heavy and ultra-heavy ions with mass $>60$ AMU (see Fig. 8 right panel, taken from Mason et al. (2004).

4. Ionization states of heavy ions that increase with increasing energy, and significantly higher average ionization states compared with the solar wind (Klecker et al. 2006).

5. Timing of suprathermal electrons that indicates source regions located in the upper corona, while the associated ions with higher ionization states need to be stripped if they passed through the denser lower corona (Kartavykh et al. 2008).

By making simultaneous measurements of $>25 \mathrm{keV}$ electrons and a number of ion species including ${ }^{3} \mathrm{He}$ and $\mathrm{Fe}$ over a broad energy range with far greater sensitivity, compared to 1 AU observations, in several individual impulsive SEP events, ISIS will provide powerful constraints on existing acceleration models.

\subsubsection{Diffusive Shock Acceleration at CME Shocks}

Diffusive shock acceleration (DSA) is the process by which energetic charged particles undergo acceleration through a combination of spatial transport and drift across the large plasma compression associated with a collisionless shock, such as that driven by a CME. DSA comprises first-order Fermi acceleration when applied to shocks that move along the magnetic field (parallel shocks), and shock-drift acceleration when applied to shocks that 



Fig. 9 Heavy ion energy spectra in a CME-associated SEP event are fitted with Ellison and Ramaty (1985) spectral form for differential intensity $j=j_{0} E^{-\gamma} \exp \left(-E / E_{0}\right)$. All elements have the same spectral index of $\gamma=1.3$ (Mewaldt et al. 2005). Spectra of different elements are offset for clarity. (Right): Values of the e-folding energies $E_{0}$ versus the ion's $\mathrm{Q} / \mathrm{M}$ ratio. Fits to the values of $E_{0}$ yield a $(\mathrm{Q} / \mathrm{M})^{1.75}$ dependence, which according to Li et al. (2009) is appropriate for a strong quasi-parallel shock

move nearly normal to the magnetic field (quasi-perpendicular shocks) (e.g., Lee 2005). Although the basic physics involved in these processes is well understood, several factors can contribute and cause the large variability that is often seen in key properties such as peak intensities, time-intensity profiles, energy spectra and composition of SEP events. Some important factors are variable seed populations (Mason et al. 1999), geometry of the shock (Tylka and Lee 2006), the presence or absence of a preceding CME from the same active region (Gopalswamy et al. 2004), scattering by ambient turbulence or by selfgenerated Alfvén waves during acceleration and transport (Giacalone 2005; Ng et al. 2003; Mason et al. 2012), and the presence of flare accelerated material (Cane et al. 2006).

A complete understanding of the contributions from each of these processes is needed to answer the following questions:

1. Why are CMEs with the same speed associated with peak proton intensities that vary by four orders in magnitude (Kahler et al. 2000)?

2. Why do CMEs with nearly the same kinetic energies produce SEP events having vastly different amounts of energy in accelerated particles (Mewaldt et al. 2008a)?

3. Why do some SEP events exhibit spectral breaks that vary strongly as a function of the ion's charge-to-mass (Q/M) ratio (see Fig. 9 and Mewaldt et al. 2005)?

4. What roles do self-excited Alfvén waves play during intense SEP events and how do they affect the streaming limits (Reames and Ng 1998; Ng et al. 2003)?

5. Do preceding CMEs generate turbulence in their wakes and/or produce suprathermal seed populations that affect particle acceleration by following CMEs (Gopalswamy et al. 2012)?

6. What factors determine the maximum energies in SEP events and why are only a small fraction of CMEs associated with the so-called Ground Level Events (GLEs) (Mewaldt et al. 2012a)?

CME shocks weaken and expand as they propagate through the inner heliosphere, therefore their properties, the conditions, and the seed populations they encounter close to the Sun 
are likely to be vastly different than those further out in interplanetary space. It is perhaps because of this that comparisons between $1 \mathrm{AU}$ data and predictions of shock acceleration models have been unsatisfactory (e.g., van Nes et al. 1984; Desai et al. 2004). As SPP moves into the region where the CME-shock acceleration processes are most efficient and the selfexcited Alfvén waves should become more prevalent, it will also identify the seed populations that are available and sample the physical environment into which different CMEs are launched. Simultaneously, ISIS will measure SEP heavy ion spectra and composition over a broad energy range between $\sim 10 \mathrm{keV} /$ nuc to $\sim 100 \mathrm{MeV} /$ nuc. These observations will facilitate direct comparisons with $\mathrm{Q} / \mathrm{M}$-dependent fractionation effects and predictions of various shock acceleration models, thereby providing answers to fundamental questions concerning SEP acceleration at CME shocks.

\subsection{Disentangle the Role of Transport}

\subsubsection{Impulsive SEP Events}

Flare particles accelerated on closed field lines require access to open field lines to escape into the heliosphere (in the absence of cross-field transport). These escaping impulsive SEPs sometimes propagate out to Earth-orbit without being scattered (see e.g., Mason et al. 1989; Haggerty and Roelof 2002). The scatter-free propagation provides accurate information about the release times of the SEPs, and also yields powerful diagnostics of the magnetic field configuration near the source regions and of the conditions under which the acceleration and escape occur. Among the mechanisms that could allow the flare-accelerated particles to escape are interchange reconnection (Wang et al. 2006) between open and closed field lines, which can occur at neutral points (Fisk 1996), and even more frequently at component reconnection sites at coronal hole boundaries (Rappazzo et al. 2012). Perpendicular diffusion (Matthaeus et al. 2003) and field-line random walk (Giacalone et al. 2000) also contribute to escape. Particles in individual SEP events that escape via reconnection are also affected by field-line meandering, as empty flux tubes (flux dropouts) are occasionally observed in between filled ones (see Fig. 10 and Mazur et al. 2000). Thus, time-intensity variations during impulsive SEP events may reflect the magnetic field topology near the source regions, as well as magnetic topological trapping boundaries that form in evolving interplanetary flux tubes (Ruffolo et al. 2003; Seripienlert et al. 2010). As SPP ventures closer to the Sun and observes many individual SEP events, ISIS will accurately determine the travel path-lengths and particle release times by measuring the onset times of ions and electrons with vastly different speeds, and by using pitch-angle distributions to pinpoint the arrival directions and the source locations. These along with other supporting measurements allow SPP to determine the coronal magnetic field configuration and conditions under which impulsive SEPs are accelerated, transported, trapped, and released into the interplanetary medium.

\subsubsection{Large Gradual SEP Events}

Particles accelerated in large gradual SEP events undergo diffusive transport from the Sun to Earth, which affects properties such as spectral breaks, time-intensity profiles, and abundance variations. However, these scattering and transport processes are poorly understood because these effects are mixed with those that result from variations in the suprathermal seed populations, CME shock properties and acceleration efficiencies, and other magnetically influenced transport effects that may be correlated with shocks (Tessein et al. 2013). 

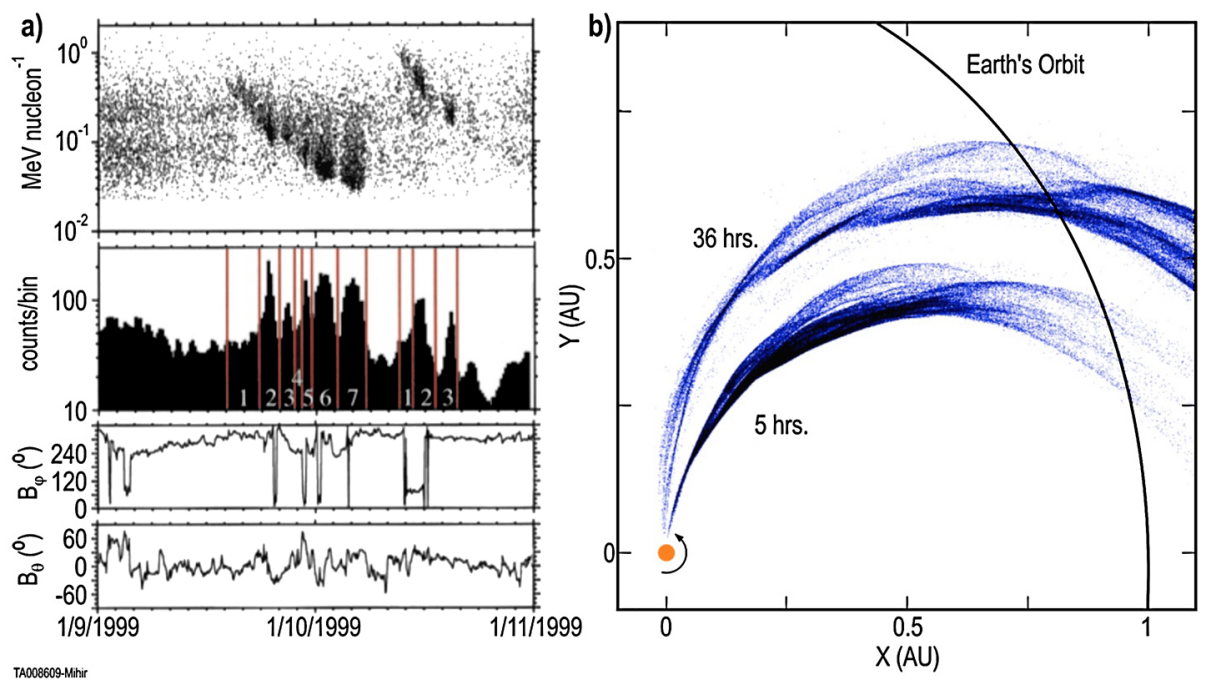

Fig. 10 Left: Energy of $\mathrm{H}-\mathrm{Fe}$ ions vs. arrival time at $1 \mathrm{AU}$ for two impulsive SEP events showing flux dropouts (from Mazur et al. 2000). Right: Results of a simulation illustrating particle positions projected onto the ecliptic plane at two different times during an impulsive SEP event. Particles are allowed to diffuse along their field line. Groups of field lines were allowed to meander at their base (from Giacalone et al. 2000)

Nonetheless, temporal variations in the abundance ratios (e.g., Fe/O) have provided powerful clues about the Q/M-dependent scattering during interplanetary transport (Tylka et al. 1999; Mason et al. 2006, 2012). Figure 11 shows an example of how the Fe/O ratio in a CME-shock associated SEP event is affected by scattering. The left panel shows that the Fe/O ratio at the same kinetic energy-per-nuc ( $\sim 30 \mathrm{MeV} /$ nuc) exhibits dramatic variations; it is close to $\sim 1$ at the start of the event and then drops to $<0.1$ around the shock arrival time. This effect led some researchers (e.g., Cane et al. 2006) to suggest that this large gradual SEP event was dominated by flare-accelerated material at the onset and later by shock-accelerated material. However, as shown in the right panel, these variations completely vanish and the $\mathrm{Fe} / \mathrm{O}$ ratio becomes nearly constant when the $\sim 30 \mathrm{MeV} /$ nuc Fe intensity is compared with the normalized $\mathrm{O}$ intensity at roughly twice the kinetic energy-per-nucleon. The same effect is observed in many large SEP events and is now attributed to Q/M-dependent scattering of particles as they propagate through the corona and the interplanetary medium (Mason et al. 2012). Nonetheless, the details of where and how this scattering process occurs, and why similar effects are not observed in all large SEP events, are not well understood. By making such measurements in many SEP events that occur along its trajectory at various radial distances, ISIS will determine how and where scattering effects start dominating SEP properties.

\subsection{Additional Science Opportunities}

In addition to obtaining required measurements of energetic electrons, ions from $\mathrm{H}$ through $\mathrm{Fe}$, and He isotopes, the EPI-Hi telescope designs lend themselves to additional important measurements with no modification of the hardware. These measurements provide additional science opportunities by separating ${ }^{20} \mathrm{Ne}$ and ${ }^{22} \mathrm{Ne}$ isotopes and by enabling serendipitous observations of energetic neutral atoms (ENAs) from large eruptions on the Sun. 



Fig. 11 Left: Fe and $\mathrm{O}$ intensity-time profiles during the November 21, 2003 large SEP event at $\sim 35 \mathrm{MeV} /$ nuc. Right: Time-intensity profiles of $\mathrm{Fe}$ and $\mathrm{O}$ are compared with $\mathrm{O}$ at $\sim$ twice the kinetic energy of Fe (adapted from Mason et al. 2006, 2012)

Interactions of accelerated ions and electrons with the solar atmosphere produce emissions that include gamma rays and neutrons, which can be uniquely identified in EPI-Hi coincidence measurements (see Sect. 4.9). Solar neutron intensities will be orders of magnitude greater than near Earth because most solar neutrons decay long before they reach 1 AU. The response of HET to secondary neutrons produced by SEP interactions in spacecraft material will be calibrated against $1-200 \mathrm{MeV}$ proton intensities measured in LET and HET. SPP's proximity to the Sun greatly benefits these observations. The direct detection of neutrons will be complementary to other measurements that EPI-Hi makes of the charged products of neutron decay.

In the 6 December 2006 solar event, which occurred at E79², the STEREO/LET instruments observed $\mathrm{H}$ ENAs arriving from within $\pm 10^{\circ}$ of the Sun hours before energetic ions arrived (Mewaldt et al. 2009). EPI-Hi may similarly detect ENAs from the charge-exchange of protons accelerated by shocks in the solar corona in cases where ENAs arrive earlier than the accelerated ions, which can occur, for example, when the spacecraft is poorly connected to the acceleration region.

The ${ }^{22} \mathrm{Ne} /{ }^{20} \mathrm{Ne}$ ratio in SEP events varies by a factor $\sim 4$ from one solar event to another (Leske et al. 2007) because of charge-to-mass (Q/M) dependent fractionation during solar energetic particle acceleration and/or transport. Thus, by assuming that both isotopes have the same mean ionic charge, we can use ${ }^{22} \mathrm{Ne} /{ }^{20} \mathrm{Ne}$ measurements to determine the extent of $\mathrm{Q} / \mathrm{M}$ fractionation and, in some cases, obtain insight into the origins of event-to-event variations of elemental abundance ratios such as $\mathrm{Fe} / \mathrm{O}$.

\subsection{ISIS Science Requirements and Performance}

ISIS provides comprehensive measurements of the energy spectra, anisotropy, and composition of suprathermal and solar energetic ions from $\sim 0.02-200 \mathrm{MeV} / \mathrm{nuc}$, as well as the energy spectra and arrival direction of $\sim 0.025-6 \mathrm{MeV}$ electrons (energy ranges indicate expected performance, see Fig. 12). The ISIS driving requirements were identified in the original ISIS proposal to NASA and have been vetted and agreed to through the development of various SPP Requirements documents. By combining measurements with other SPP instruments and from instruments on other spacecraft, ISIS addresses key questions 
Fig. 12 Ion energy spectra of different inner heliospheric particle populations that SPP will encounter and the required energy range coverage for EPI-Lo (green) and EPI-Hi (blue) as well as the broader expected performance (lighter shades) for ISIS' overall energy coverage. Also shown is the energy range coverage for electrons

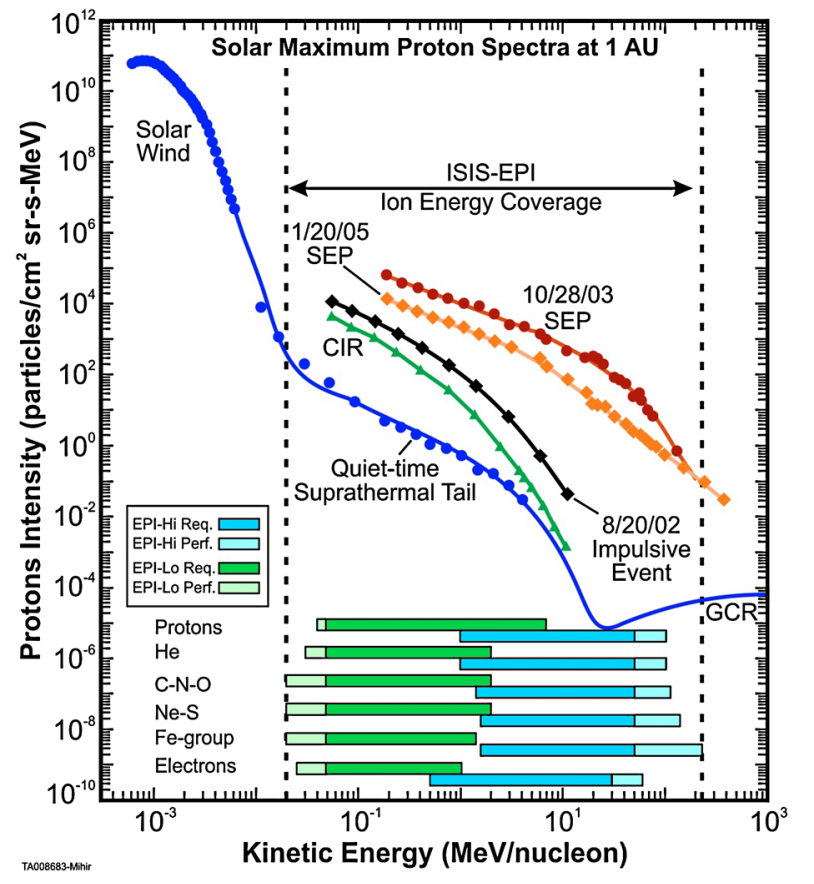

\section{(1)}
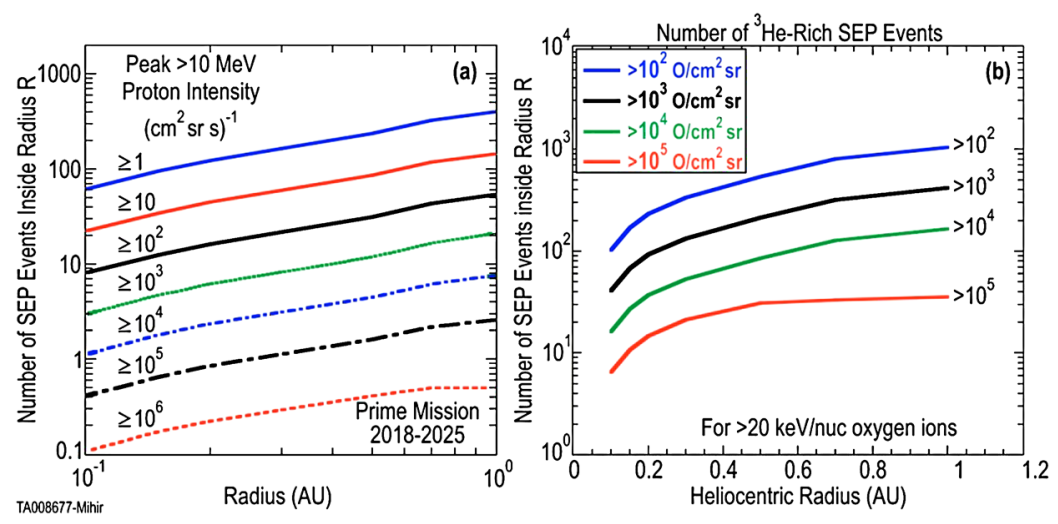

Fig. 13 Number of gradual (left) and impulsive (right) SEP events during the SPP prime mission inside a given heliocentric radius as a function of event size. These plots include the relative amount of time spent at each radial distance. The estimates for gradual events are based on NOAA/GOES data from 1976-2008, an assumed 11-year solar cycle, and a radial gradient of $R^{-2.4}$ based on Lario et al. (2006). Estimates for impulsive ${ }^{3} \mathrm{He}$-rich events are based on 1998-2006 data from ACE/ULEIS, measurements of impulsive electron events by Wind/3DP (Wang 2010), and on an assumed fluence radial gradient of $R^{-2}$

concerning the origin, acceleration, and transport of different types of inner heliospheric particle populations, including those associated with CIRs, ACRs, and GCRs. During the course of the SPP mission, ISIS will measure a sufficient number of impulsive and gradual SEP events (see Fig. 13) to meet all of its scientific objectives. Driving requirements for key instrument functional parameters are provided in Table 2; we note that while meeting these 
Table 2 Measurement requirements for ISIS-EP suite

\begin{tabular}{ll}
\hline Functional parameter & Measurement requirements \\
\hline Energy range & $e-:<0.05$ to $>3 \mathrm{MeV}$ \\
& $p+$ /ions: $<0.05$ to $>50 \mathrm{MeV}$ \\
Energy binning (resolution) & $>6$ bins/decade \\
Cadence & $e-:<1 \mathrm{~s}$ for selected electron rates \\
& $p+$ /ions: 5 s for selected ion rates \\
Field of view & $>\pi / 2$ ster coverage in both sunward and anti-sunward hemispheres, \\
& including coverage within 10 degrees of the nominal Parker spiral field \\
& direction at perihelion \\
Angular sectoring & $e-:<45^{\circ}$ sectors \\
& $p+/$ ions: $<30^{\circ}$ sectors \\
Composition & At least $\mathrm{H}, \mathrm{He},{ }^{3} \mathrm{He}, \mathrm{C}, \mathrm{O}, \mathrm{Ne}, \mathrm{Mg}, \mathrm{Si}, \mathrm{Fe}$ \\
Max intensity $<1 \mathrm{MeV}$ & $>10^{6}$ particles cm ${ }^{-2} \mathrm{sr}^{-1} \mathrm{~s}^{-1}$ \\
Max intensity $>1 \mathrm{MeV}$ & $>5 \times 10^{5}$ particles cm ${ }^{-2} \mathrm{sr}^{-1} \mathrm{~s}^{-1}$ \\
\hline
\end{tabular}

requirements is adequate to achieve all of our scientific objectives, the expected performance is even better.

Figure 13 shows that the numbers of gradual and impulsive SEP events expected to be observable with EPI-Hi during the SPP prime mission, as a function of particle fluence and heliocentric radius, are more than sufficient to address all the science questions discussed above and to enable great discovery science on the SPP mission.

\section{ISIS Suite Overview}

\subsection{Introduction}

The ISIS Energetic Particle Suite measures energetic electrons, protons, and heavy ions across a broad range of energies. To provide this wide coverage, ISIS includes two instruments that contain multiple sensors with detectors optimized for various parts of the energy measurement range. The suite combines EPI-Lo and EPI-Hi mounted together on the ISIS Bracket (Fig. 14). ISIS is mounted at the aft end of the ram side of the SPP spacecraft providing an open FOV toward the direction of the nominal Parker spiral over much of the solar encounter phase, while staying within the umbra of the SPP Thermal Protection System (TPS).

EPI-Lo (Fig. 15) measures energetic ions from $0.02 \mathrm{MeV} /$ nuc to $\sim 15 \mathrm{MeV}$ total energy and energetic electrons from $25-1000 \mathrm{keV}$. To provide a large FOV with hemispherical coverage, EPI-Lo has eight wedges. Each wedge has 10 apertures that collimate energetic particles into pathways through the electro-optics, which allows their speed and energy to be measured while also registering which aperture they passed through. The eight sensor wedges are serviced by an electronics box that contains the Event Board and the Power Board. The Event Board contains the analog and digital processing circuits to record the events and communicates to the spacecraft through command and telemetry channels. The Power Board contains both the low voltage power converters as well as the high voltage power required for the sensors. 
Fig. 14 ISIS suite

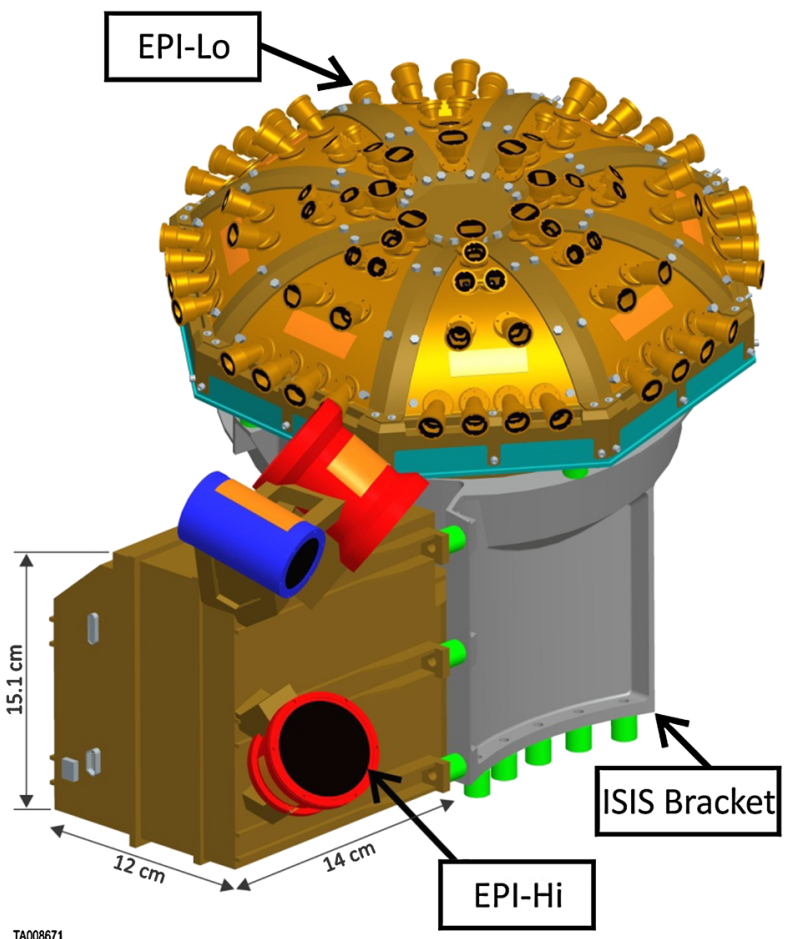

Fig. 15 EPI-Lo mechanical design

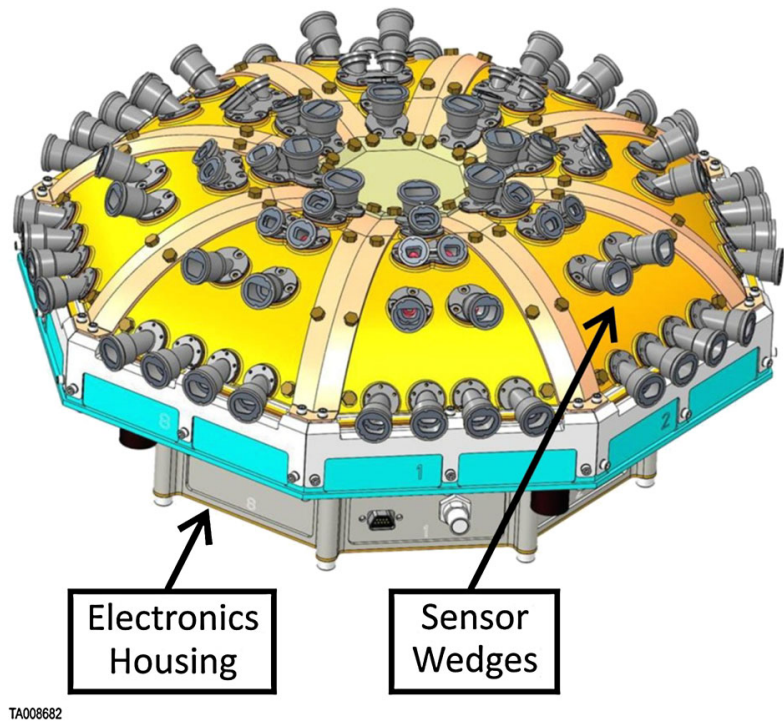

EPI-Hi (Fig. 16) measures energetic protons and He nuclei from $\sim 1$ to $\sim 100 \mathrm{MeV} /$ nuc (and higher energies for heavier elements) and energetic electrons from $\sim 0.5$ to $\sim 6 \mathrm{MeV}$. To cover this energy range, and to provide wide FOV coverage, EPI-Hi has three telescopes, a double-ended high energy telescope (HET), a double-ended low energy telescope (LET1), 
Fig. 16 EPI-Hi mechanical design

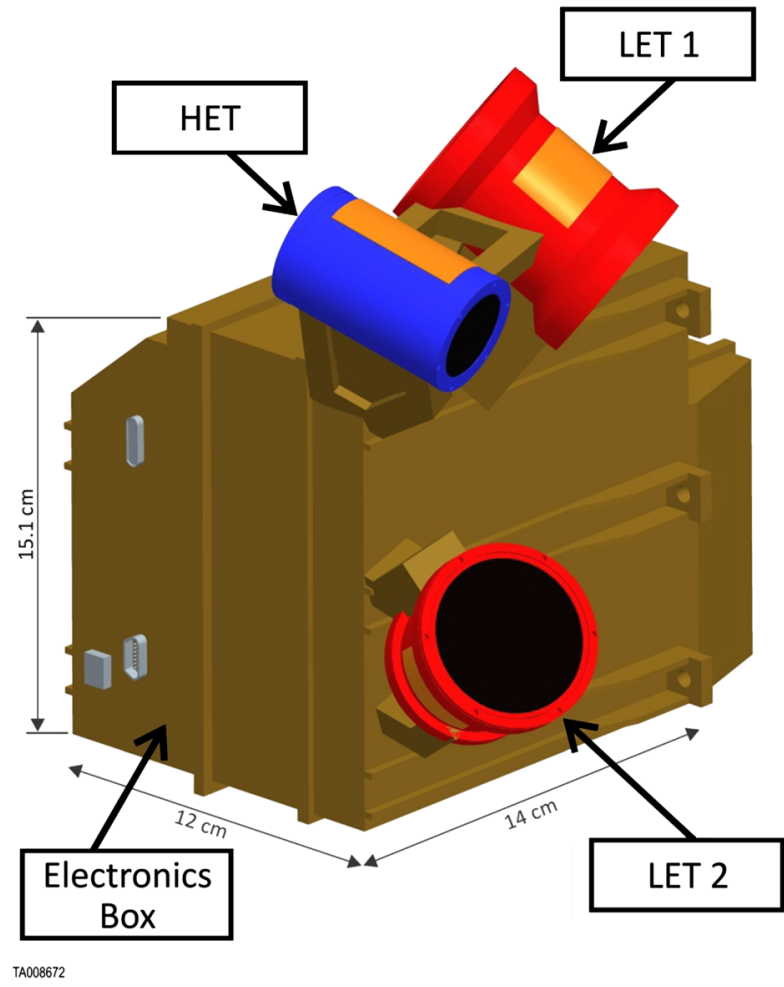

and a single-ended low energy telescope (LET2). These telescopes are mounted on the EPIHi Electronics Box, which contains an analog and digital processing electronics board for each telescope; a detector bias power supply; a digital processing unit for the instrument to coordinate its operations and communicate by command and telemetry channels with the spacecraft; and a low voltage power supply.

\subsection{ISIS Fields of View}

\subsubsection{Location on Spacecraft}

ISIS is located as far aft on the spacecraft body as possible, on the ram side, just inside the umbra line (Fig. 17). This provides protection from direct solar heating, but still allows ISIS to view within $10^{\circ}$ of the Sun-Probe line, thereby providing access to the nominal direction of the Parker Spiral magnetic field over much of the solar encounter phase.

\subsubsection{FOV Maps}

Because ISIS must remain in the umbra, its FOV has several blockages from the spacecraft itself. The TPS is the predominant FOV blockage (see Figs. 18 and 19). At the extremes of ISIS' FOV, there are small blockages due to the deployed solar arrays and the solar limb sensors. The EPI-Hi FOVs are a series of five overlapping $45^{\circ}$ half-angle cones, three of which are provided by the low energy telescopes (the double-ended LET1, and the singleended LET2). The other two $45^{\circ}$ half-angle cones are provided by the double-ended, higher 
Fig. 17 Location of ISIS on SPP spacecraft

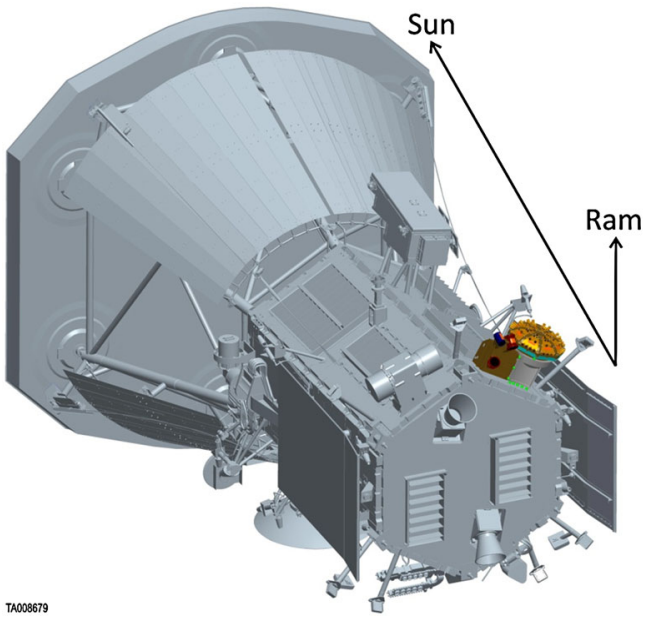

Five $45^{\circ}$ - Half-Angle View Cones

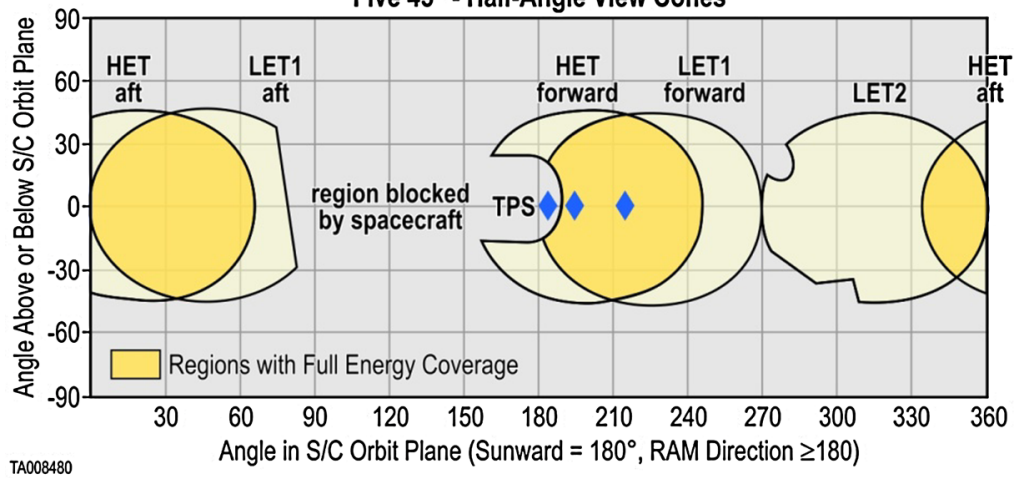

Fig. 18 EPI-Hi FOV Map. Three blue diamonds indicate the locations of the average Parker spiral magnetic field for a solar wind velocity of $400 \mathrm{~km} / \mathrm{s}$ at heliocentric distances of $0.05,0.25$, and $0.7 \mathrm{AU}$ (left to right)

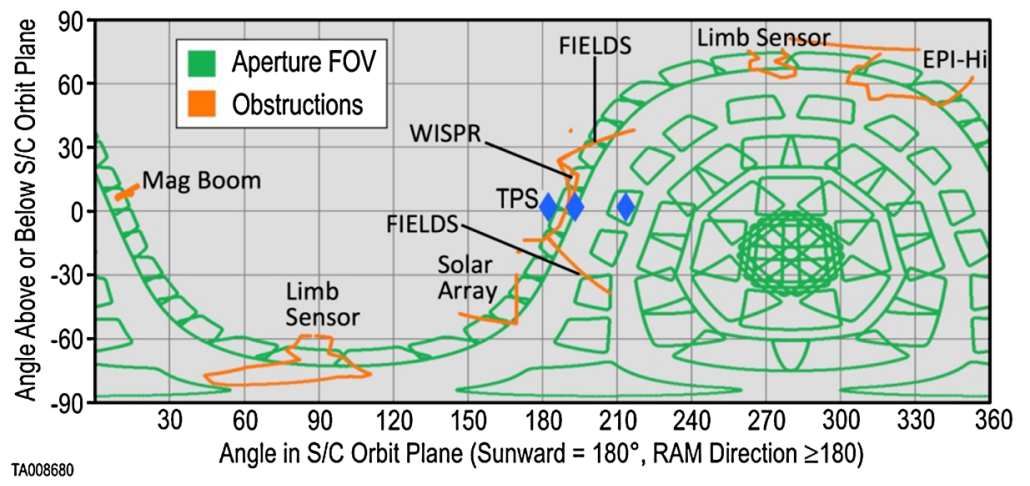

Fig. 19 EPI-Lo FOV Map. Three blue diamonds indicate the locations of the average Parker spiral magnetic field for a solar wind velocity of $400 \mathrm{~km} / \mathrm{s}$ at heliocentric distances of $0.05,0.25$, and $0.7 \mathrm{AU}$ (left to right) 
energy telescope (HET). The EPI-Hi telescope FOVs are overlapped to provide full energy coverage in the sunward and anti-sunward direction in two, nearly-complete $45^{\circ}$ half-angle cones. Further coverage is provided in the directions that are not blocked by the spacecraft by the low energy telescopes alone. The EPI-Lo FOV is an array of 80 apertures, ten on each of eight wedges. These apertures are arranged to sample a hemispherical FOV that includes viewing in the sunward and anti-sunward hemispheres as well as coverage near the direction of the nominal Parker spiral.

\subsection{ISIS Bracket}

The ISIS suite is integrated onto a single, combined ISIS bracket. The bracket is designed to allow flexibility and position the ISIS instruments as close to the allowable umbra-line as possible since the final position of the SPP TPS may be shifted slightly during spacecraft Integration and Testing (I\&T). The nominal bracket design holds ISIS just within the allowed limit with $\sim 2^{\circ}$ safety margin to the actual umbra line, which keeps the ISIS FOVs as close as possible to the Sun-Probe line. However, the SPP spacecraft center-of-mass must be very close to the center-of-pressure on the TPS in order to minimize pointing perturbations. To do this, the TPS, which is initially oversized, will be trimmed to its final configuration during spacecraft I\&T. By design, the TPS will not be trimmed below the limit we have designed to, but it could be trimmed less, which would move the actual umbra line farther away from ISIS and reduce viewing toward the Sun-Probe line. To prevent this reduction in our FOV in this key region of interest, the ISIS bracket is designed to accommodate a late TPS modification by extending the suite farther away from the spacecraft panel, thus restoring ISIS to the position as close as possible to the umbra.

\subsection{Spacecraft Accommodation}

\subsubsection{Mass, Power, and Telemetry}

ISIS has been optimized for the SPP mission. Mass is the most constrained resource for the SPP spacecraft, and power is second because of the mass penalty for cooling the solar arrays. The ISIS team has worked diligently to remain within limits set by the mission design. The resource estimates come from a detailed Master Equipment List in which the estimates for each item are carefully maintained and revised over the course of development. Given the heritage of the electronics, the power and telemetry estimates have remained steady during the course of the project development to date. To minimize overall SPP risk, the ISIS team was given a small mass increase to reduce development risks during the risk-reduction SPP activities in Phase B. The Current-Best-Estimates (CBEs) as of the time of the ISIS and mission PDRs, as well as uncertainty estimates are listed in Table 3.

\subsubsection{Thermal Design}

On a spacecraft that is designed to fly much closer to the Sun than any other mission in history, one might reasonably expect that the thermal environment would be extreme and that the limiting hot-case for the thermal design would be at perihelion. However, the limiting hot-case for the thermal design of ISIS is actually near $1 \mathrm{AU}$, just after launch when the SPP spacecraft has to perform various maneuvers that allow direct solar illumination of ISIS. After those early operations, ISIS remains in the umbra of the TPS at all other times, which provides a stable environment that is "in-family" with past, heritage missions for this type of instrumentation. 
Table 3 ISIS resource table

\begin{tabular}{|c|c|c|c|c|c|c|c|c|c|c|}
\hline & \multicolumn{3}{|l|}{ EPI-Hi } & \multicolumn{3}{|c|}{ EPI-Lo } & \multicolumn{3}{|c|}{ ISIS bracket } & \multirow{2}{*}{$\begin{array}{l}\text { ISIS } \\
\text { Total }\end{array}$} \\
\hline & $\mathrm{CBE}$ & $\begin{array}{l}\text { Uncer- } \\
\text { tainty }\end{array}$ & Total & $\mathrm{CBE}$ & $\begin{array}{l}\text { Uncer- } \\
\text { tainty }\end{array}$ & Total & $\mathrm{CBE}$ & $\begin{array}{l}\text { Uncer- } \\
\text { tainty }\end{array}$ & Total & \\
\hline Mass [kg] & 3.628 & 0.692 & 4.320 & 3.435 & 0.656 & 4.091 & 0.817 & 0.156 & 0.973 & 9.384 \\
\hline $\begin{array}{l}\text { Instrument } \\
\text { power [W] }\end{array}$ & 5.810 & 0.960 & 6.770 & 4.170 & 0.830 & 5.000 & NA & NA & NA & 11.770 \\
\hline $\begin{array}{l}\text { Operational } \\
\text { heaters [W] }\end{array}$ & 0.480 & 0.070 & 0.550 & NA & $\mathrm{NA}$ & NA & NA & NA & NA & 0.550 \\
\hline \multirow{3}{*}{$\begin{array}{l}\text { Survival } \\
\text { heaters [W] }\end{array}$} & \multirow[t]{3}{*}{3.810} & 0.570 & 4.380 & 2.450 & 0.370 & 2.820 & NA & NA & NA & 7.200 \\
\hline & & & & \multicolumn{2}{|c|}{ EPI-Hi } & & \multicolumn{3}{|l|}{ EPI-Lo } & ISIS \\
\hline & & & & $\mathrm{Ra}$ & Burst & Total & Raw & Burst & Total & Total \\
\hline \multicolumn{4}{|c|}{ Uncompressed data volume [Gbit/orbit] } & 3.6 & 0.000 & 3.660 & 11.320 & 0.200 & 11.520 & 15.180 \\
\hline \multicolumn{4}{|c|}{ Compressed data volume (75\%) [Gbit/orbit] } & 2.7 & 0.000 & 2.745 & 8.490 & 0.200 & 8.690 & 11.435 \\
\hline \multicolumn{4}{|c|}{ Packetized data volume (105\%) [Gbit/orbit] } & 2.8 & 0.000 & 2.882 & 8.915 & 0.210 & 9.125 & 12.007 \\
\hline
\end{tabular}

Data volume formula: $105 \%[75 \% *($ EPI-Hi Raw + EPI-Lo Raw $)+$ EPI-Hi Burst + EPI-Lo Burst $]$

The thermal design of the SPP mission requires that the instruments be thermally isolated from the SPP spacecraft. The ISIS bracket configuration provides thermal isolation from the spacecraft deck, from the instruments, between the instruments, and from the bracket, thermally isolating the instruments themselves. This is accomplished by including 0.5 in $(1.27 \mathrm{~cm})$ ULTEM $^{\circledR}$ spacers at all of the mounting bolt locations on both sides of the bracket. To provide electrical conductivity, while still maintaining thermal isolation, thin straps of copper bridge the gap. These provide a very small cross-section to minimize thermal conductivity, but have a large surface area for good, high-frequency electrical grounding. Multi-layer insulation (MLI) covers the majority of ISIS, except for the apertures and thermal radiators, to minimize radiant heat transfer. EPI-Hi and EPI-Lo have independent survival heaters controlled by the spacecraft, and EPI-Hi also has an operational heater controlled by the instrument itself.

\subsubsection{Electrical Interfaces}

EPI-Hi and EPI-Lo have completely independent electrical interfaces to the SPP spacecraft. Each has separate command and telemetry interfaces to the SPP Command and Data Handling $(\mathrm{C} \& D H)$ unit as well as separate power interfaces for instrument power to the SPP Power Distribution Unit (PDU). The SPP spacecraft also provides independent temperature sensors and survival heaters that are used to control the EPI-Hi and EPI-Lo temperatures.

Each instrument has separate A-side/B-side command and telemetry interfaces. Low Voltage Differential Signaling (LVDS) is used to establish a high-speed serial communication protocol with Interface Transfer Frames (ITFs) as the packet format. Command ITFs are used to send spacecraft time and status as well as instrument-command packets to each instrument. ISIS uses the spacecraft time and status information to configure itself autonomously. The instruments return instrument-status and telemetry packets in Telemetry ITFs. The instrument-status can be interpreted on-board the spacecraft to make real-time autonomy decisions. The telemetry packets are stored on the SPP data recorder for down-link 
to ground stations at the appropriate time. To maintain time synchronization, a 1 Pulse-PerSecond (PPS) signal is provided by the spacecraft. Instead of using a separate line, a "virtual PPS" is provided by carefully controlling the timing of the start-bit of the first byte of the Command ITFs.

EPI-Hi and EPI-Lo have independent power connections to the SPP PDU, each with their own switches. At the SPP mission level (including measurements from the other instruments), mission success can be achieved with either EPI-Hi or EPI-Lo, so additional power redundancy is not required. The spacecraft provides a standard $+28 \mathrm{~V}$ power bus. ISIS uses a common Low Voltage Power Supply (LVPS) design, which meets the Electromagnetic Interference/Compatibility (EMI/EMC) requirements of the SPP Mission. The LVPS design is customized for each instrument and provides reverse voltage protection, in-rush current limiting, EMI filtering, and isolation between the spacecraft power return and the ground returns of the secondary power supply rails that are developed within each instrument.

\subsubsection{Alignment and Fields of View Blockages}

The alignment requirements of ISIS are relatively straightforward to achieve. We require an overall accuracy of $1^{\circ}$ alignment between ISIS' and the spacecraft's coordinate systems. This is achieved through close tolerances on mounting holes. The budget for the tolerances is distributed through each of the components and tracked by systems engineering at the ISIS level. Besides our alignment with the spacecraft, we also require knowledge of blockages in our FOV. These are primarily caused by the TPS, the solar arrays, and the solar limb sensors. As described in Sect. 2.3, ISIS' FOV is sensitive to the final location of the TPS and we have made provisions to adjust the ISIS bracket in response to shifts in the TPS during spacecraft $\mathrm{I} \& \mathrm{~T}$.

\subsubsection{Nitrogen Purge}

In order to maximize the performance and lifetime of the detectors within ISIS, high-purity gaseous nitrogen (GN2) purge must be maintained during instrument I\&T, during spacecraft I\&T, and up until launch. Before integration onto the SPP spacecraft, ISIS Ground Support Equipment (GSE) is used to provide purge. Once integrated to the spacecraft, purge is provided through the flight purge lines built into the spacecraft. The instrument designs include provisions for venting to prevent over-pressure due to the purge and during launch.

\subsubsection{Covers}

In order to protect the sensitive apertures of the instruments, the ISIS team provides "redtag" remove-before-flight covers. During I\&T, these covers remain installed except for specific times for tests that require that they be removed. In those cases, special procedures must be used to protect the apertures. No launch covers are required. The ability to withstand the launch environment without covers will be qualified and verified through component-level Engineering Model (EM) and full Flight Model (FM) testing.

\subsubsection{Safe Arm Plug}

The EPI-Lo instrument has a red-tag remove-before-flight Safe Plug that limits high voltage within the instrument to air-safe levels. This red-tag Safe Plug is removed before full high voltage in vacuum testing and before launch. The EPI-Hi instrument does not require a Safe Plug because the detector voltages and conductor spacing is air-safe even at full voltage levels. 


\subsection{Science Data and Operations}

\subsubsection{ISIS Science Operations Center}

The ISIS Science Operations Center (SOC) processes, distributes, and archives all ISIS data. This integrated approach provides an efficient and effective method for verifying all data products and coordinating and combining data not just between EPI-Lo and EPI-Hi, but with data from the other SPP instrument teams as well as ancillary data.

The ISIS SOC coordinates the development of instrument command sequences based on science activity plans developed across the ISIS team. The ISIS SOC is tasked with verifying and validating the command sequences and coordinating with the SPP Mission Operations Center (MOC) to send the commands to the SPP spacecraft. It also maintains command and telemetry databases and develops processing pipelines using algorithms generated by the ISIS team. The health and safety of the instruments are monitored, and alerts to the team are issued if alarms are raised. The housekeeping and science data are processed and distributed within the team and to the broader community on a timely basis to support the SPP mission. Thoroughly vetted and validated data products will be transmitted to the NASA-designated repository for permanent archiving.

\subsubsection{Integrated Science Team}

The ISIS science team includes scientists from the ISIS Leadership, EPI-Hi, EPI-Lo, and SOC groups as well as Senior Science Mentors and theory and modeling team members who are leading experts in energetic particle science (see Table 1). These scientists are integrated into the on-going instrument development process as well as the operations planning and data analysis efforts. The ISIS science team works in close coordination with the other SPP instrument investigation teams. It also coordinates data collection and analysis with other missions (e.g. Solar Orbiter) and ground-based observations. This close coordination inside the ISIS science team and cooperation with the broader science community provides a truly outstanding integrated science investigation of the Sun.

\section{Energetic Particle Instrument-Low Energy (EPI-Lo)}

EPI-Lo measures energetic particles in the lower portion of the ISIS energy range. As discussed in Sect. 2, the two ISIS instruments complement one another in their energy ranges and their sky coverage in order to obtain the comprehensive set of observations needed to understand solar energetic particle sources, acceleration and transport close to the Sun.

\subsection{EPI-Lo Overview}

EPI-Lo is a novel, light-weight, high-heritage, time-of-flight (TOF) based, mass spectrometer that measures energetic electron $(25-1000 \mathrm{keV})$ and ion $(\sim 0.04-7 \mathrm{MeV}$ for protons and $\sim 0.02-2 \mathrm{MeV} /$ nuc for heavier ions) spectra and resolves all major heavy ion species and ${ }^{3} \mathrm{He}$ and ${ }^{4} \mathrm{He}$ over much of this energy range in multiple directions. ISIS thus covers the critical energy range from suprathermal energies ( $\sim 20 \mathrm{keV} / \mathrm{nuc})$ up to the lower portion of the EPI-Hi energy range with a single instrument. The EPI-Lo characteristics and projected performance are summarized in Table 4.

EPI-Lo (Figs. 15 and 20) consists of eight sensor wedges mounted above an electronics box. It has 80 separate entrances (10 on each of eight wedges) densely sampling over half 
Table 4 EPI-Lo instrument required and projected performance

\begin{tabular}{|c|c|c|c|}
\hline Parameter & Required & Goal (expected) & Comment/Heritage \\
\hline Electron energies & $50-500 \mathrm{keV}$ & $25-1000 \mathrm{keV}$ & $\begin{array}{l}\text { Electron capability from } \\
\text { JEDI, RBSPICE }\end{array}$ \\
\hline Ion energies & $\begin{array}{l}50 \mathrm{keV} / \text { nuc- } \\
15 \mathrm{MeV} \text { Total } E\end{array}$ & $\begin{array}{l}\sim 20 \mathrm{keV} / \text { nuc- } \\
15 \mathrm{MeV} \text { Total } E \\
\sim 85 \mathrm{MeV} \text { Total } E \text { for Fe }\end{array}$ & $\begin{array}{l}\text { Capability based on } \\
\text { that of RBSPICE. } \\
\text { Maximum energy } \\
\sim 1.5 \mathrm{MeV} / \text { nuc for } \mathrm{Fe}\end{array}$ \\
\hline Energy resolution & $\begin{array}{l}45 \% \text { for required } \\
\text { energy range }\end{array}$ & $\begin{array}{l}11 \% \text { for required } \\
\text { energy range }\end{array}$ & Telemetry limited \\
\hline Time sampling & $5 \mathrm{sec}$ & $1 \mathrm{sec}$ & $\begin{array}{l}\text { Telemetry and/or } \\
\text { statistics limited }\end{array}$ \\
\hline Angle resolution & $<30^{\circ} \times<30^{\circ}$ & $\begin{array}{l}\text { Ions, } \sim 15^{\circ} \times 12^{\circ} \text { to } \\
<30^{\circ} \times<30^{\circ} e-, 45^{\circ}\end{array}$ & Varies with elevation \\
\hline $\begin{array}{l}\text { Pitch Angle (PA) } \\
\text { coverage }\end{array}$ & $\begin{array}{l}0^{\circ}-90^{\circ} \text { or } 90^{\circ}-180^{\circ} \text {, } \\
\text { some samples in both } \\
\text { hemispheres }\end{array}$ & $\begin{array}{l}0^{\circ}-90^{\circ} \text { or } 90^{\circ}-180^{\circ} \text {, } \\
\text { some samples in both } \\
\text { hemispheres }\end{array}$ & \\
\hline Time for full PA & $1-5 \mathrm{sec}$ & $1-5 \mathrm{sec}$ & Telemetry limited \\
\hline Ion composition & $\begin{array}{l}\mathrm{H},{ }^{3} \mathrm{He},{ }^{4} \mathrm{He}, \mathrm{C}, \mathrm{O}, \mathrm{Ne} \\
\mathrm{Mg}, \mathrm{Si}, \mathrm{Fe}\end{array}$ & $\begin{array}{l}\mathrm{H},{ }^{3} \mathrm{He},{ }^{4} \mathrm{He}, \mathrm{C}, \mathrm{O}, \mathrm{Ne} \\
\mathrm{Mg}, \mathrm{Si}, \mathrm{Fe}\end{array}$ & $\begin{array}{l}{ }^{3} \mathrm{He} /{ }^{4} \mathrm{He} \sim 50 \text { to } \\
1000 \mathrm{keV} / \text { nuc }\end{array}$ \\
\hline $\begin{array}{l}\text { Electron } \\
\text { sensitivity, } \\
\text { geometric factor, } \\
\text { counting rate, and } \\
\text { background drivers }\end{array}$ & $\begin{array}{l}<10^{6} \mathrm{~cm}^{-2} \mathrm{~s}^{-1} \mathrm{sr}^{-1} \\
\left(G \sim 0.05 \mathrm{~cm}^{2} \mathrm{sr}\right. \\
\text { measure event rates to } \\
>50 \mathrm{kHz})\end{array}$ & $\begin{array}{l}10^{2}-10^{7} \mathrm{~cm}^{-2} \mathrm{~s}^{-1} \mathrm{sr}^{-1} \\
\left(G>0.05 \mathrm{~cm}^{2} \mathrm{sr} ;\right. \\
\text { measure event rates to } \\
>700 \mathrm{kHz} \text {; background } \\
\text { rate }<1 \mathrm{~Hz})\end{array}$ & $\begin{array}{l}G=\text { Geometric factor } \\
\left(\mathrm{cm}^{2} \mathrm{sr}\right) 8 \text { pixels/sensor; } \\
\text { background rate is } \\
\text { spectral-slope dependent }\end{array}$ \\
\hline $\begin{array}{l}\text { Ion sensitivity, } \\
\text { geometric factor, } \\
\text { counting rate, and } \\
\text { background drivers }\end{array}$ & $\begin{array}{l}10^{1}-10^{6} / \mathrm{cm}^{-2} \mathrm{~s}^{-1} \mathrm{sr}^{-1} \\
\left(G \sim 0.05 \mathrm{~cm}^{2} \mathrm{sr} ;\right. \\
\text { measure event rates to } \\
>50 \mathrm{kHz} \text {; at minimum } \\
\text { intensity require } \\
\text { accidental rate } \\
<\sim 50 \mathrm{kHz})\end{array}$ & $\begin{array}{l}10^{0}-10^{7} / \mathrm{cm}^{-2} \mathrm{~s}^{-1} \mathrm{sr}^{-1} \\
\left(G>0.07 \mathrm{~cm}^{2} \mathrm{sr}\right. \\
\text { measure event rates to } \\
>700 \mathrm{kHz} \text {; at minimum } \\
\text { intensity require } \\
\text { accidental rate } \\
<\sim 5 \mathrm{kHz})\end{array}$ & 80 pixels/sensor \\
\hline
\end{tabular}

of the sky. This configuration permits full angular distributions without articulation or duty cycle, and allows for measuring the first-arriving, field-aligned ions at the spacecraft for a broad range of vector magnetic (B) field directions.

Understanding coronal acceleration processes requires ion-mass resolution sufficient to measure separately the intensities of the isotopes ${ }^{3} \mathrm{He}$ and ${ }^{4} \mathrm{He}$ (Fig. 21), and the elements $\mathrm{C}$, $\mathrm{O}, \mathrm{Ne}, \mathrm{Mg}$, Si, and $\mathrm{Fe}$, while simultaneously providing angular coverage with at least $45 \%$ energy resolution.

EPI-Lo achieves this resolution, including separation of ${ }^{3} \mathrm{He}$ from ${ }^{4} \mathrm{He}$, over most of its angular coverage from $200 \mathrm{keV}$ to $4 \mathrm{MeV}$ total particle energy. EPI-Lo also returns highresolution energy spectra (64 log-spaced energy bins) at reduced time resolution (necessitated by downlink limitations) and measures the required ion composition and pitch angle distributions from $30 \mathrm{keV} / \mathrm{nuc}$ to $0.3-1.0 \mathrm{MeV} /$ nuc every 5 or $30 \mathrm{~s}$. In this way ion acceleration histories are completely characterized with no uncertainty owing to an insufficient energy range or composition misidentification. EPI-Lo rejects background by requiring coincidence between the start and stop pulses for the TOF measurement, along with an energy 

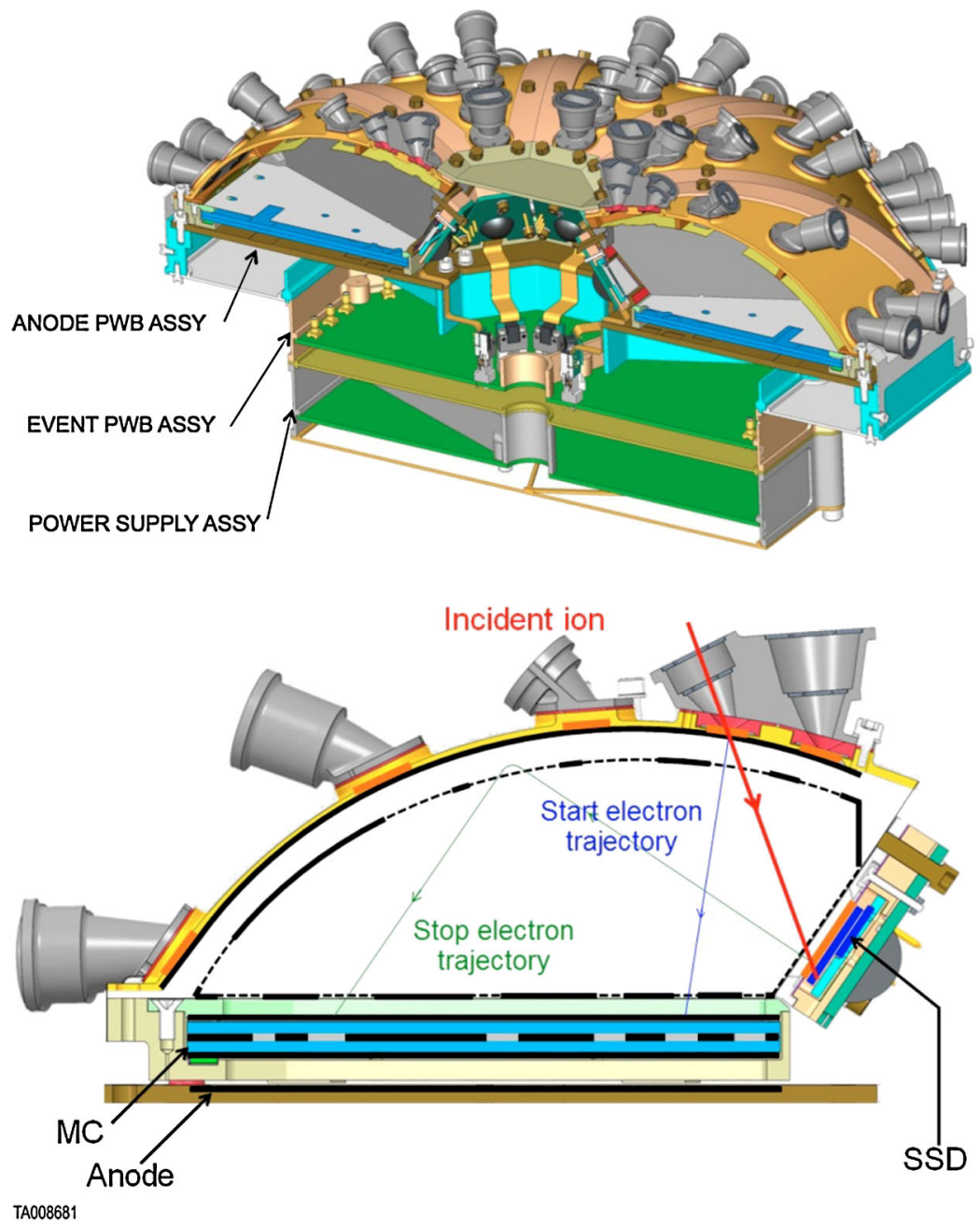

Fig. 20 EPI-Lo comprises eight wedges mounted on a common electronics box. Ions generate start/position electrons as they transit thin foils in the entrance apertures, and then strike a stop foil and solid-state detector (SSD), yielding angle and $E \times$ TOF. Energetic electrons enter the same apertures as the ions, and are detected in a second set of SSDs located behind light- and ion-rejecting cover foils

measurement between appropriate TOF time gates. This rejection is non-linear, and is very effective (resulting in inconsequential rates of false valid events) for background counting rates in individual detectors (singles rates) below $\sim 10^{6} \mathrm{~s}^{-1}$; projected singles rates from combined background and foreground sources are $\leq 3 \times 10^{5} \mathrm{~s}^{-1}$. Incoming ion velocities are determined by measuring the TOF between two thin (100 nm Start, $65 \mathrm{~nm}$ Stop) carbonpolyimide-aluminum (and palladium, Start only) foils. An ion passing through each of the foils produces secondary electrons, which are deflected toward a microchannel plate (MCP) producing "Start" and "Stop" pulses. The ion entrance angles are determined from the position where the Start electrons strike the MCP and are unique for each entrance foil location. The ion energy deposited in the SSD, together with velocity from the TOF, determines species through an onboard table lookup. 
Fig. 21 Representative species separation for EPI-Lo holes 2 $\left(22.5^{\circ}\right.$ from the instrument normal) and 5 (the holes about the instrument perimeter, $90^{\circ}$ from instrument normal) based on measured TOF resolution performance on test model. Data taken using an alpha source in hole 2 is overlaid on the hole 2 simulated data. ${ }^{3} \mathrm{He}$ is well distinguished from ${ }^{4} \mathrm{He}$ at 1:100 abundance ratio to $\geq 1.5 \mathrm{MeV} /$ nuc. The best performance for species identification is in the 64 holes at the 3,4 and 5 positions, at $45^{\circ}$, $67.5^{\circ}$, and $90^{\circ}$ from the instrument normal

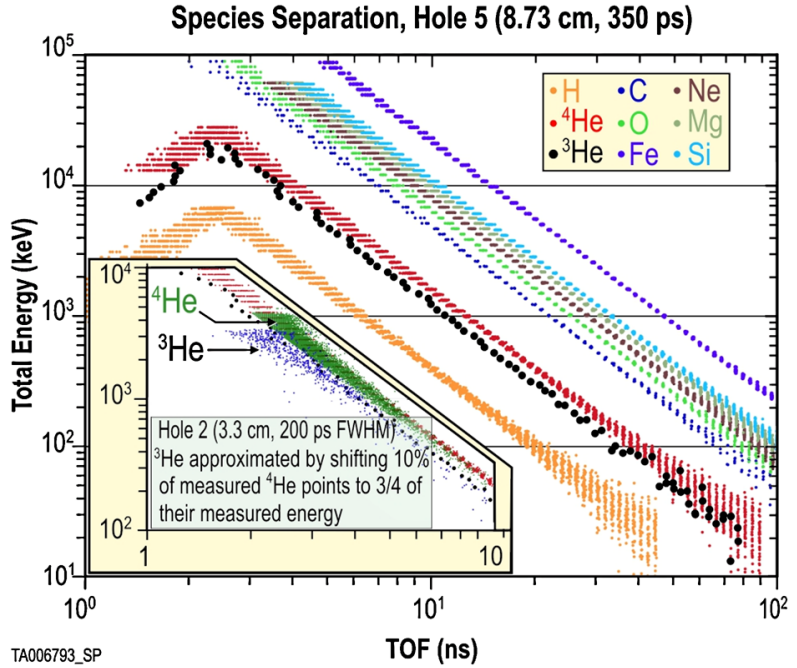

EPI-Lo also detects electrons from $\sim 25 \mathrm{keV}$ to $1000 \mathrm{keV}$. EPI-Lo uses solid-state detectors (SSDs) shielded by aluminum flashing of $\sim 2 \mu \mathrm{m}$ thickness as also used in multiple current and upcoming missions, e.g., Cassini-MIMI (Krimigis et al. 2004), MESSENGER EPS (Andrews et al. 2007), New Horizons PEPSSI (McNutt et al. 2008), Juno JEDI (Mauk et al. 2013), Van Allen Probes RBSPICE (Mitchell et al. 2013), and MMS EIS (Mauk et al. 2014). The relatively thick aluminum flashing naturally suppresses light, which is a very important feature for this intrinsically single parameter measurement. While the primary electron measurement does not identify which entrance aperture an electron enters through, each EPI-Lo wedge contains independent electron SSDs, so the sector of the sky is identified over an angular coverage similar to that for the ions. For the small subset of the electrons that produce secondary electrons as they transit the entrance foils, an additional mode that requires a Start pulse along with the SSD pulse identifies the specific entrance aperture that the electron entered through. This mode also provides better background rejection.

MCP detectors and SSD's have been optimized for use on this mission. We also produced a prototype wedge (Fig. 22), which has been used for extensive testing and development of the EPI-Lo concept and design. Finally, lead development engineers and instrument scientists on JEDI, RBSPICE, and EIS are all participating in the EPI-Lo effort, as well the corresponding leads that developed and continue to operate PEPSSI.

\subsection{EPI-Lo Expected Performance}

The detectors in EPI-Lo are sensitive to charged particles and to light. The MCPs are sensitive to ultraviolet (UV) light, including both EUV and shorter far ultraviolet (FUV) wavelengths whereas the SSDs are sensitive to everything from X-rays to visible wavelengths. However, the mechanisms are different. MCPs respond to charged particles impacting their surface, and UV produces secondary electrons (photoelectrons) on surfaces (either the surface of the MCP itself, or the Start and Stop foils designed to produce secondary electrons as ions penetrate them, or other surfaces inside the sensor volume), which can then enter channels and be amplified similarly to signals from any other particle.

SSDs respond to particles as they deposit energy in the bulk material of the detector. They free bound electrons to create electron-hole pairs and so increase conductivity. In SSDs, light 
Fig. 22 EPI-Lo wedge prototype

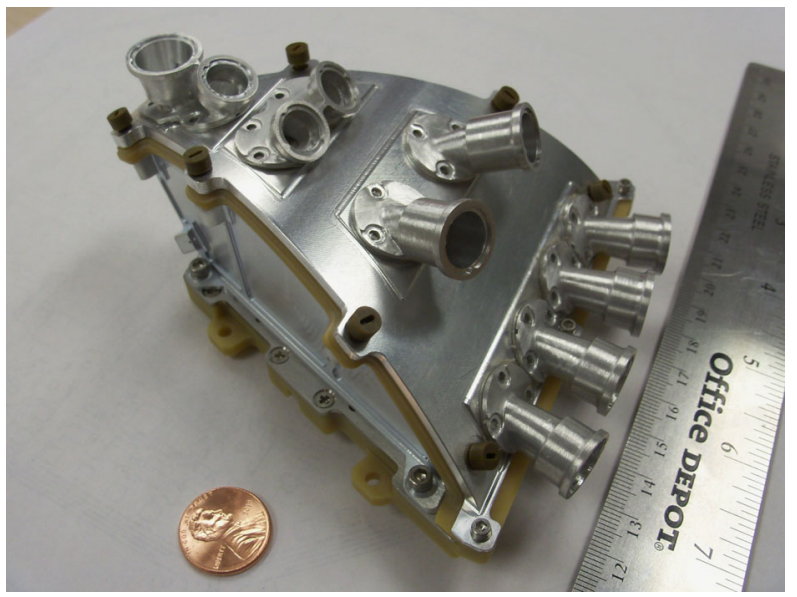

can also energize bound electrons sufficiently that they become free and increase conductivity. Energetic X-rays may deposit enough energy to create free electrons that then raise the energy of sufficient numbers of bound electrons so that the resulting current spike is above threshold. Lower energy photons will not do so, but enough of them arriving within an SSD event time constant ( $\sim 1$ microsecond) can free sufficient numbers of electrons to register as events, or to raise the electronic noise of the detector unacceptably.

In both cases, light (both UV and visible) must be reduced to a level where these effects cannot degrade the particle measurements. Controlling the amount of light entering the sensor volume involves a variety of techniques: (1) eliminating stray-light leak paths into the instrument; (2) collimating the entrance apertures to eliminate exposure to trajectories that are not useful for particle analysis; (3) coating surfaces from which light may be reflected into the detectors with low-reflectance coatings; (4) using high-work-function surfaces in locations where UV photons may strike surfaces from which photoemission is undesirable; (5) designing the Start and Stop foils using materials and thicknesses that reflect and/or filter the UV and visible light, reducing the light that can directly enter the sensor volume to acceptable levels; (6) taking precautions against failure of this filtering approach as a consequence of small numbers of pinholes in the foils (either from dust impact or launch vibration damage); and, (7) designing the sensor timing constraints such that controlled rates of photoelectron production from UV photons do not impact particle measurements.

Briefly, the instrument design carefully considers: (1) stray light paths and avoids them; (3) coating surfaces as a second-order consideration relative to direct paths for light and UV; and (7) timing constraints fundamental to the instrument design: an approach success-


Horizons, Van Allen Probes, Juno, and MMS as well as the ENA imagers on Cassini and IMAGE).

All of these programs also rely on collimation and filtering by foils, and all were subject to pinhole degradation at various levels, so these considerations are not new; however, the

\footnotetext{
${ }^{1}$ The Energetic Particle Spectrometer (EPS) on the MErcury Surface, Space ENvironment, GEochemistry, and Ranging (MESSENGER) mission to Mercury, the Pluto Energetic Particle Spectrometer Science Investigation (PEPSSI) on the New Horizons mission to Pluto, the three JEDI instruments on the Juno mission to Jupiter, the Radiation Belt Storm Probes Ion Composition Experiment (RBSPICE) on the twin Van Allen Probes in the Earth's radiation belts and the Energetic Ion Spectrometer (EIS) on the Magnetospheric Multiscale mission (MMS) spacecraft to be launched into Earth orbit.
} 
Fig. 23 Intensity of Ly- $\alpha$ EUV vs. solar elongation. Most EPI-Lo entrances include angles no closer than 20 degrees elongation (which implies a maximum Ly- $\alpha$ intensity of $\left.\sim 10^{9} \mathrm{~cm}^{-2} \mathrm{~s}^{-1} \mathrm{sr}^{-1}\right)$. For four apertures close to the TPS, the maximum is

$\sim 10^{12} \mathrm{~cm}^{-2} \mathrm{~s}^{-1} \mathrm{sr}^{-1}$, but average only $\sim 10^{10} \mathrm{~cm}^{-2} \mathrm{~s}^{-1} \mathrm{sr}^{-1}$

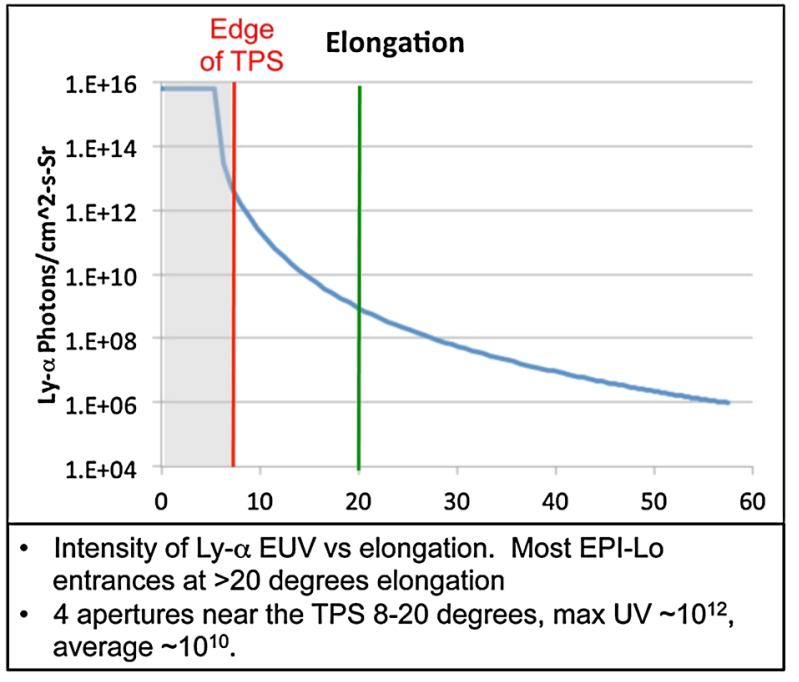

SPP environment is sufficiently unique that careful consideration to these approaches is required for EPI-Lo.

The environment in which EPI-Lo will operate is one never before experienced. It has been extensively modeled, and there is considerable knowledge of the light environment. The SPP team has provided their best estimate of the worst-case photon environment as a function of solar elongation angle at perihelion. This, and the other expected SPP environments are specified in an internal Project-provided environmental design and test requirements document. Light at the ISIS location is dominated by Thomson-scattered photons, to first order a process that preserves the shape of the solar spectrum. The intensity of the scattered light is a fairly strong function of elongation angle, dominated primarily by the line-of-sight integrated electron density, which varies with elongation (Fig. 23). While dustscattered light dominates at large elongations it is much weaker than the Thomson-scattered light at smaller elongations, so the foil filtering design required by the Thomson-scattered light at smaller elongations is more than adequate for the dust-scattered light.

We know the elongation angles of the various apertures on EPI-Lo. In considering these, we treat the EPI-Lo apertures in two distinct groups: those that contain sky elements within $\sim 12^{\circ}$ elongation angle (the edge of the TPS is at about $8^{\circ}$ ), and those that do not. For those with elongation angles less than $12^{\circ}$, the rough order-of-magnitude (ROM) for the UV and light environment is approximately equivalent to having the disk of the Sun directly in the FOV of that aperture at $1 \mathrm{AU}$. This then means that the filtering requirements for those apertures are approximately the same as what would be required for EPI-Lo at $1 \mathrm{AU}$ with the Sun directly in the aperture FOV.

Several approaches contribute to determining the required filtering properties of the foils. For visible light (which primarily concerns the SSDs), we have used flight experience from various instruments in Earth orbit. For example, the ISEE-1 Energetic Particle Detector (EPD) had $30 \mu \mathrm{g} / \mathrm{cm}^{2}$ of $\mathrm{Al}$ covering its SSD, and suffered from visible light contamination when the detector viewed the Sun directly. The IMP-8 EPD had $40 \mu \mathrm{g} \mathrm{cm}^{-2}$ of Al covering its SSD and did not respond to direct Sun in the visible, but it did respond to solar X-ray events. From this we conclude that $40 \mu \mathrm{g} \mathrm{cm}^{-2}$ of $\mathrm{Al}$ or the equivalent would be sufficient for EPI-Lo for elongations $<12^{\circ}$. For these small elongation foils we are baselining a Start foil of $24 \mu \mathrm{g} \mathrm{cm}^{-2}$ of Al plus $18 \mu \mathrm{g} \mathrm{cm}^{-2}$ of palladium (Pd), which combined with the Stop foil 


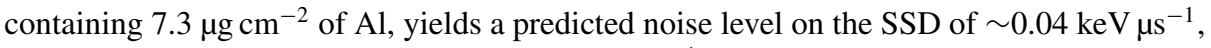
well below the electronic noise level of $\sim 7 \mathrm{keV}_{\mu \mathrm{s}}{ }^{-1}$.

For the larger elongation apertures we have baselined a total of $7.3 \mu \mathrm{g} \mathrm{cm}{ }^{-2}$ of Al plus $18 \mu \mathrm{g} \mathrm{cm}^{-2}$ of Pd on the Start foil, and $7.3 \mu \mathrm{g} \mathrm{cm}^{-2}$ of Al on the Stop foil. The predicted noise level for this combination is $0.7 \mathrm{keV} \mathrm{\mu s}^{-1}$ in the SSD. We plan to test at this level, and increase the thickness of the aluminum layers if necessary. It should be noted that these are ROM estimates and not really directly comparable. The Thomson scattered light is diffuse, whereas sunlight at $1 \mathrm{AU}$ is collimated. Appropriate descriptions in terms of photons at a particular wavelength are number of photons $\mathrm{cm}^{-2} \mathrm{~s}^{-1}$ for sunlight at 1 AU but number $\mathrm{cm}^{-2} \mathrm{~s}^{-1} \mathrm{sr}^{-1}$ for the scattered light at $10 \mathrm{R}_{\mathrm{S}}$ ( $\sim$ perihelion for SPP). The way we employ our model is by using the EPI-Lo geometric factor for the apertures, which lie within a given elongation angle range we are modeling, and calculating the number of photons $\mathrm{s}^{-1}$ that hit the aperture foils, so the calculation is correspondingly conservative. We then run the filter model with a fraction of the full Sun at $1 \mathrm{AU}$ determined such that we match that number of photons/s on the same aperture foils.

The foils employed as filters result in energy loss of ions entering the instrument before they reach the TOF section and subsequently the Stop foil and SSD. These energy losses and straggling affect the minimum energy and energy resolution at the lower end of the EPI-Lo energy range. However, based on TRIM and GEANT4 simulations, as well as experience with similar foils on previous instruments, the required foils will still permit EPI-Lo to provide its full, required measurements.

We calculated the transmission of visible light through $\mathrm{Al}$ and $\mathrm{Pd}$. Aluminum is quite efficient at filtering visible light, but palladium is not, so we rely primarily on Al for filtering in the visible wavelength range with palladium included to filter short-wavelength EUV.

For UV transmission we have employed a filter model that we have maintained throughout the development of the Cassini INCA and IMAGE HENA, instruments and all of the "hockey pucks," which incorporates the solar EUV spectrum from about 10 to $140 \mathrm{~nm}$ and uses Henke atomic scattering tables (http://henke.lbl.gov/optical_constants/asf.html). This model has been quite successful in modeling the expected responses of foil-based time-offlight by energy (TOF $\times E$ ) instruments to the UV environments of interplanetary space, Earth, Jupiter, and Saturn. Applying this model to the UV environment provided by the SPP team, the ISIS team determined that the predicted Start foil rates for an EPI-Lo quadrant from UV photo-electrons is $\sim 1 \times 10^{3} \mathrm{~s}^{-1}$. The predicted Stop rate is also $\sim 1 \times 10^{3} \mathrm{~s}^{-1}$. The predicted accidental TOF $\times E$ rate is $\ll 1.0 \mathrm{~s}^{-1}$ for the quadrant and, hence, not significant.

The foil materials are sufficiently thick that neither photoelectrons nor solar wind electrons (nor solar wind ions) will penetrate them. However, particles can enter through holes in the foils (either pre-existing, from launch, or produced by dust impacts in flight), so we also calculate and account for the susceptibility to backgrounds from photoelectrons and solar wind ions and electrons. The highest photoelectron intensities will be in the vicinity of the Sun-facing surface of the spacecraft TPS where the plasma sheath is predicted to have a thickness ${ }^{2}$ on the order of centimeters, therefore those electrons will not reach EPI-Lo (which is in the umbra, $\sim 3$ meters from the TPS edge). EPI-Lo must be able to tolerate the photoelectron flux produced by the UV flux striking surfaces relatively close to the EPI-Lo

\footnotetext{
${ }^{2}$ The Debye length sets the scale length for the plasma sheath. With respect to the solar wind, the electron temperature is expected to have a characteristic temperature of $\sim 100 \mathrm{eV}$ and at $10 \mathrm{R}_{\mathrm{S}}$, the density should be typically no more than $\sim(215 / 10)^{2} \times 10 \mathrm{~cm}^{-3} \sim 4600 \mathrm{~cm}^{-3}$; the corresponding Debye length is $\sim 1$ meter. Photoelectron temperatures are much less $\sim 1 \mathrm{eV}$, and the densities will be much larger, so the sheath thickness will be much less near the sunlit parts of the spacecraft.
} 
apertures, and therefore umbral UV intensities. Those intensities are small (both in number flux and in energy) relative to solar wind electron intensities, therefore we consider only the latter.

Solar wind electron flux at perihelion is expected to rise to $\sim 2 \times 10^{12} \mathrm{~cm}^{-2} \mathrm{~s}^{-1}$ (with a typical thermal energy of $\sim 100 \mathrm{eV}$; numbers provided by the SPP Project). Dividing by $2 \pi$ sr, this amounts to about $3.2 \times 10^{11} \mathrm{~cm}^{-2} \mathrm{~s}^{-1} \mathrm{sr}^{-1}$. Solar wind electrons entering through pinholes in the EPI-Lo aperture foils at relatively low energy $(\leq 100 \mathrm{eV})$ will behave like Start electrons inside the instrument; they will be focused onto the MCP areas that register Start events. These need to be limited to less than $\sim 1 \times 10^{6} \mathrm{~s}^{-1}$, preferably much less, but at this level the instrument will still function as designed; for example, the RBSPICE instruments on the Van Allen Probes mission routinely run with Start rates of 2 to $4 \times 10^{6} \mathrm{~s}^{-1}$ and still return well-behaved, calibrated TOF $\times E$ data.

The largest solid angles viewed by the Start foils are $\sim 0.2$ sr, so those foils would see $\sim 6 \times 10^{10}$ solar wind electrons $\mathrm{cm}^{-2} \mathrm{~s}^{-1}$. If we wish to limit the intensity to $\sim 10^{6} \mathrm{~s}^{-1}$ for a quadrant, this requires that the total pinhole area through the 20 foils (of the quadrant, there being 10 apertures to the octant) be $\sim 2 \times 10^{-5} \mathrm{~cm}^{2}$. The foil support grids are 180 lines/inch, or $\sim 70$ lines $/ \mathrm{cm}$. This corresponds to a grid element width of $1 / 70 \mathrm{~cm}$, or $143 \mu \mathrm{m}$. The grid wires are $12 \mu \mathrm{m}$ in width, so a grid element has an area of $\sim 130 \times 130 \mu \mathrm{m}^{2}$, or about $1.7 \times 10^{-4} \mathrm{~cm}^{2}$. This implies that with only one grid-element sized pinhole in any one of the 20 foils in a quadrant, we would already exceed our limit by about one order of magnitude $\left(\sim 6 \times 10^{10} \times 1.7 \times 10^{-4} \mathrm{~cm}^{2} \sim 10^{7} \mathrm{~s}^{-1}\right)$.

In tests of high velocity dust impacting flight-like foils at the dust facility at the University of Colorado, only micron-sized holes were produced by the dust. Therefore, even though we do not expect a dust grain to be able to take out a whole grid element, during Phase B, we decided to take an even more conservative approach and use two foils in the collimators, separated by $0.5 \mathrm{~cm}$ minimum (effectively turning a single hole into an aperture-constrained "telescope"). Then the geometric factor for pinholes in both foils is $\Omega \times 1.7 \times 10^{-4} \mathrm{~cm}^{2}$ where $\Omega$ is the solid angle defined by the two pinholes. This geometric factor is $\sim 6 \times$ $10^{-8} \mathrm{~cm}^{-2} \mathrm{sr}^{-1}$. Multiplying by $3.2 \times 10^{11} \mathrm{~cm}^{-2} \mathrm{~s}^{-1} \mathrm{sr}^{-1}$, we obtain a rate of $\sim 2 \times 10^{4} \mathrm{~s}^{-1}$. Even for one such pair of holes in every entrance of a quadrant, the total rate would amount to only $\sim 4.0 \times 10^{5} \mathrm{~s}^{-1}$, which more than meets our criterion. ${ }^{3}$

It should be pointed out that the need for double foils for mitigation of solar wind electrons does not require both foils be of the same design. We only require the collimator foils to reduce the susceptibility to pinholes of the electron-blocking capacity. Therefore, our collimator foil is much simpler than the start foil, using a single layer (1000 $\AA$ of polyimide with $50 \AA \mathrm{C}$ flashing on the inward-facing side) sufficiently thick that pinholes on such a foil supported by a 180 line-per-inch grid become very unlikely.

\subsection{EPI-Lo Electrical}

The EPI-Lo electronics box contains all the electronics to run the instrument other than the energy and timing preamps, which are located in the sensor wedges. The box contains two octagonal boards mounted into metal frames. The boards stack one on top of the other, with an internal connector providing electrical interconnects between the boards. The functional

\footnotetext{
${ }^{3}$ Work by the SPP project indicates about 0.1 hits/foil over the mission $(\sim 0.25-0.4$ hits/mission in the direction viewing the Sun). This is the geometry for a hit through both foils, but it is under the assumption that the particle will survive the first foil hit. So EPI-Lo will likely accumulate holes but at a tolerable rate.
} 


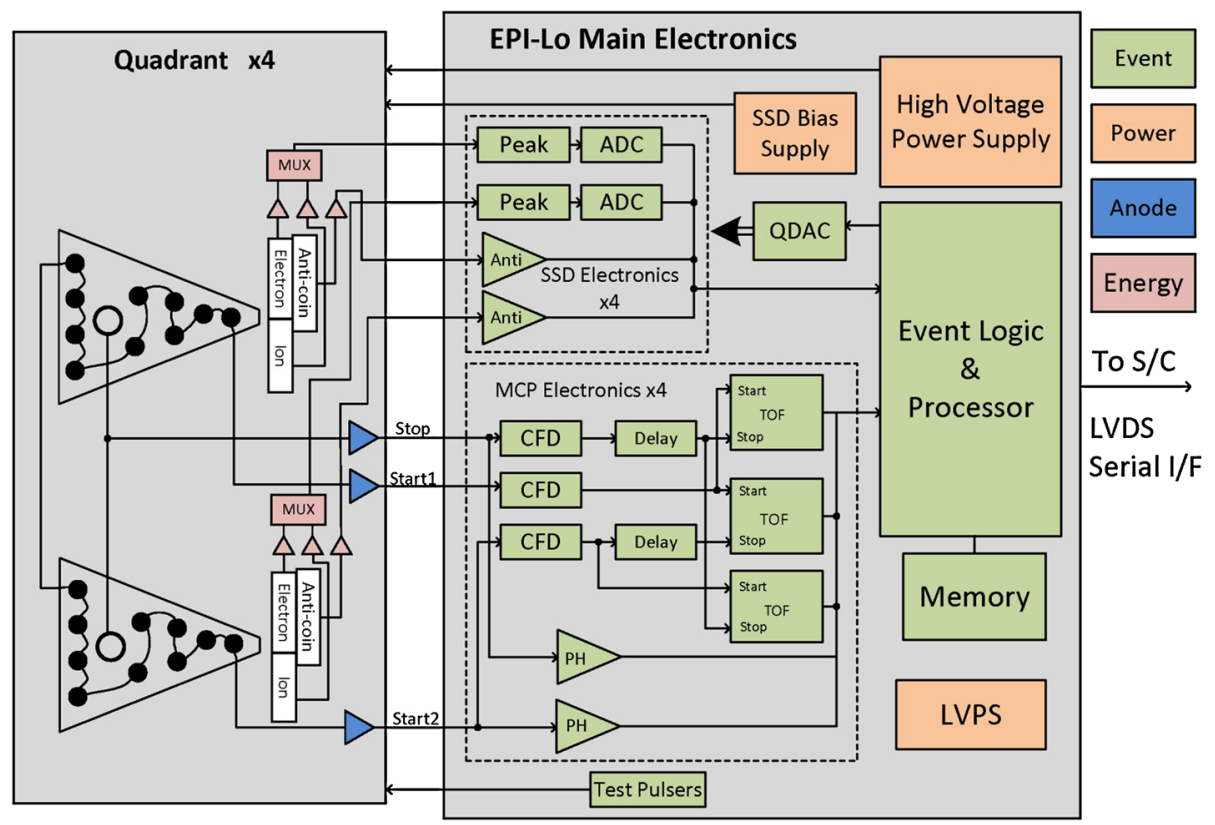

Fig. 24 EPI-Lo block diagram

breakup between the two boards minimizes the number of interconnects needed. See Fig. 24 for the block diagram.

The electronics are designed to handle solar energetic particle event intensities up to at least $10^{6}$ particles $\mathrm{cm}^{-2} \mathrm{sr}^{-1} \mathrm{~s}^{-1}$. This includes handling electron counts rates $\geq 70,000$ counts per second and total ion count rates (SSD and MCP valid coincident event) of $\geq 70,000$ counts per second. In both cases, these events can be either evenly distributed over the entire instrument or concentrated in one wedge. The term "handle" is used to mean that the incoming particles are processed in the instrument such that the particle types, directions, and rates can be determined. Note that ground software rate correction will be necessary when rates are sufficiently high (e.g. $\geq 10^{6}$ starts per second or $\geq 40,000$ total ion count rates per second). With the current mission and environment assumptions, we expect that rates will be sufficiently high only $\sim 5 \%$ of the time to require such corrections.

\subsubsection{Event Board}

An ion entering the sensor through one of the collimator apertures will deposit energy in the SSD and produce secondary electrons in the Start and Stop foils, which are amplified by the MCP and collected in distinct positions on the anode. This collection of time-correlated signals is called an event for the purpose of the description of the electronics that follows. The Event board directly processes the sensor SSD and anode preamp output signals, and contains all necessary analog and digital circuitry to process and store event information on an event-by-event basis. The energy signals from the eight SSD preamplifier sets are processed in parallel peak-detect/discriminator circuit/ADC chains. The MCP anode signals are processed via constant-fraction discriminators (CFDs) and time-to-digital conversion (TDC) circuitry; these measured time differences are converted into event look direction 
and particle velocity in the field-programmable gate array (FPGA). FPGA-based event logic also determines which signals comprise valid ion and electron events and coordinates all event hardware processing timing. A soft-core processor (i.e. a processor implemented in VHDL) is also embedded in the FPGA to provide all command, control, telemetry, and data processing functions of the instrument. SRAM, MRAM, and PROM memory storage is provided on the board to support the processor.

EPI-Lo uses a pulse-width technique to determine the energy deposited in each detector for energies above $\sim 1 \mathrm{MeV}$ (Paschalidis 2008). This method, used on the Juno JEDI, the RBSPICE, and MMS EIS, allows the energy dynamic range to be extended from $\sim 1.5 \mathrm{MeV}$ to a total energy $\sim 15-20 \mathrm{MeV}$. In order to cover fully iron composition with no gaps between EPI-Lo and EPI-Hi, the maximum energy measured will be extended to $\sim 85 \mathrm{MeV}$ for iron (1.5 MeV/nuc for Fe nuclei). Three separate approaches to this extension of the iron energy range have been identified, and the preferred approach will be finalized and tested in the engineering model prior to instrument CDR. Each sensor uses an existing, flight-qualified, application-specific integrated circuit (ASIC) containing preamplifier/shaper circuits to amplify the SSD and APD (analog peak detect) signals, shape the pulse, and generate timing triggers on the rise and fall of each pulse. These signals feed into the EPI-Lo FPGA where the coincidence logic and other digital processing begin. The EPI-Lo FPGA-based processor accumulates events into rates, and packetizes the telemetry products.

\subsubsection{Power Board}

The Power board contains both the low and high voltage power supplies. The low voltage portion takes spacecraft primary power on a single 9 pin connector and generates $1.5 \mathrm{~V}$ (for the FPGA core), $3.3 \mathrm{~V}$ (primarily for digital interface logic, memories, and TDCs), and $5 \mathrm{~V}$ (primarily for analog functions). A $15 \mathrm{~V}$ output powers the high voltage power supply (HVPS). The HVPS generates the necessary high voltage outputs for the sensor MCP and electron optics, with a maximum voltage output of $3300 \mathrm{~V}$. It can independently control high voltage to each of the four quadrants. A bias supply provides up to $250 \mathrm{~V}$ for the SSDs.

\subsubsection{Anode Board}

The four anode boards are located directly under the MCPs and each board covers two octants (sensor wedges). Each anode board (a "quadrant") has 20 start pick-up anodes configured as a delay line, and two stop pick-up anodes tied together. One pre-amplifier reads out each of the two sides of the delay line, and one pre-amplifier reads out the combined stop anodes. Locating the pre-amplifiers directly on the anode board reduces system noise.

\subsubsection{Energy Boards}

Each of the eight SSD assemblies has an energy board that contains the pre-amplifiers and shapers for each of the electron, ion, and anti-coincidence detectors. The energy board is mounted directly to the back of the SSD to minimize noise. The electron and ion SSDs have pulse height analysis electronics on the event board while the anti-coincidence detector is monitored by a comparator with a programmable threshold to give a simple yes/no particle event result. The energy board also supports test inputs, both external and from the event board, and filtering for the bias voltage. 


\subsubsection{EMI/EMC Design Considerations}

Of principal concern for EMC design are the power supplies. These are controlled to a frequency window centered at $n \times 50 \mathrm{kHz}$ with $n \geq 3$ and $500 \mathrm{ppm}$ wide overall operating conditions and time. The LVPS is synchronized to $200 \mathrm{kHz}$ by a $400 \mathrm{kHz}$ clock provided by the digital boards. EPI-Lo has a $40 \mathrm{MHz}$ oscillator and EPI-Hi has a $58.8 \mathrm{MHz}$ oscillator; both evenly divide to $400 \mathrm{kHz}$. To minimize interference, transformers and large inductors are placed as far from the box walls as possible, and stable currents are employed to minimize changes in magnetic emissions. EPI-Lo does have nickel grids, and concerns associated with those are mitigated with careful handling, use of non-magnetic tools, and testing.

All electronic parts are selected for proven radiation tolerance: total ionizing dose (TID) $>100 \mathrm{krad}$, no latch-up, and acceptable single-event upset (SEU) performance. Triple module redundancy (TMR) and error correction code (ECC) are used on vulnerable registers inside the FPGA. PROM-based boot code is used to ensure reliable memory loading and checking capabilities. Analog parts are selected with low dose effects in mind. Parts comply with Goddard Space Flight Center's (GSFC's) “EEE-INST-002” Level II requirements.

\subsection{EPI-Lo Mechanical}

EPI-Lo uses a symmetric design to enable the wide field of view in a compact, low-mass package. Parts consist of the eight wedge assemblies and their closeouts, a top close out, the "spider" frame, which holds the wedges, and the event- and power-board slices, which comprise the components of the electronics box (Fig. 25). The common wedge design is shown in Fig. 26. Each contains an MCP assembly, an SSD assembly, and a collimator set. Alignment of all of the pieces enables the coverage and functionality of the detector overall. Preliminary design analysis has been done with a finite element model (FEM) using 89,077 nodes and 70,645 elements.

The preliminary structural analysis of the baseline design was performed using MSC Nastran, MAYA SATK ${ }^{\circledR}$, and Femap for analysis. The model was simplified wherever possible to reduce solution time. Printed wiring assemblies (PWAs) were modeled as plate elements with uniform stiffness, thickness, and density, and the instrument model was oriented to the ISIS bracket configuration in relation to the spacecraft panels.

We performed a mechanical modal analysis to determine mechanical resonances. The analysis showed the first mode to be $304 \mathrm{~Hz}$ (for the Event PWA) and $553 \mathrm{~Hz}$ for the overall instrument. The analysis environmental input levels per spacecraft requirements were performed for all three orthogonal axes relative to the spacecraft panel. The 3- $\sigma$ acceleration random response enveloped the static load requirement as desired for EPI-Lo displacements, stresses, and forces. The random-vibration PWA displacement response may be relatively high for electrical, electronic, and electromechanical (EEE) part solder/lead-wire fatigue resistance, and further analysis is planned after EEE parts placement is finalized. All margins of safety are positive for the current model configuration, and a detailed analysis will be carried out for the flight configuration to confirm that the flight design has positive margins and meets the minimum frequency requirement.

\subsection{EPI-Lo Software}

EPI-Lo software consists of instrument Common Software (reused from other projects), SPP Host Software, EPI-Lo Application Software, and EPI-Lo Boot Software. The Com- 

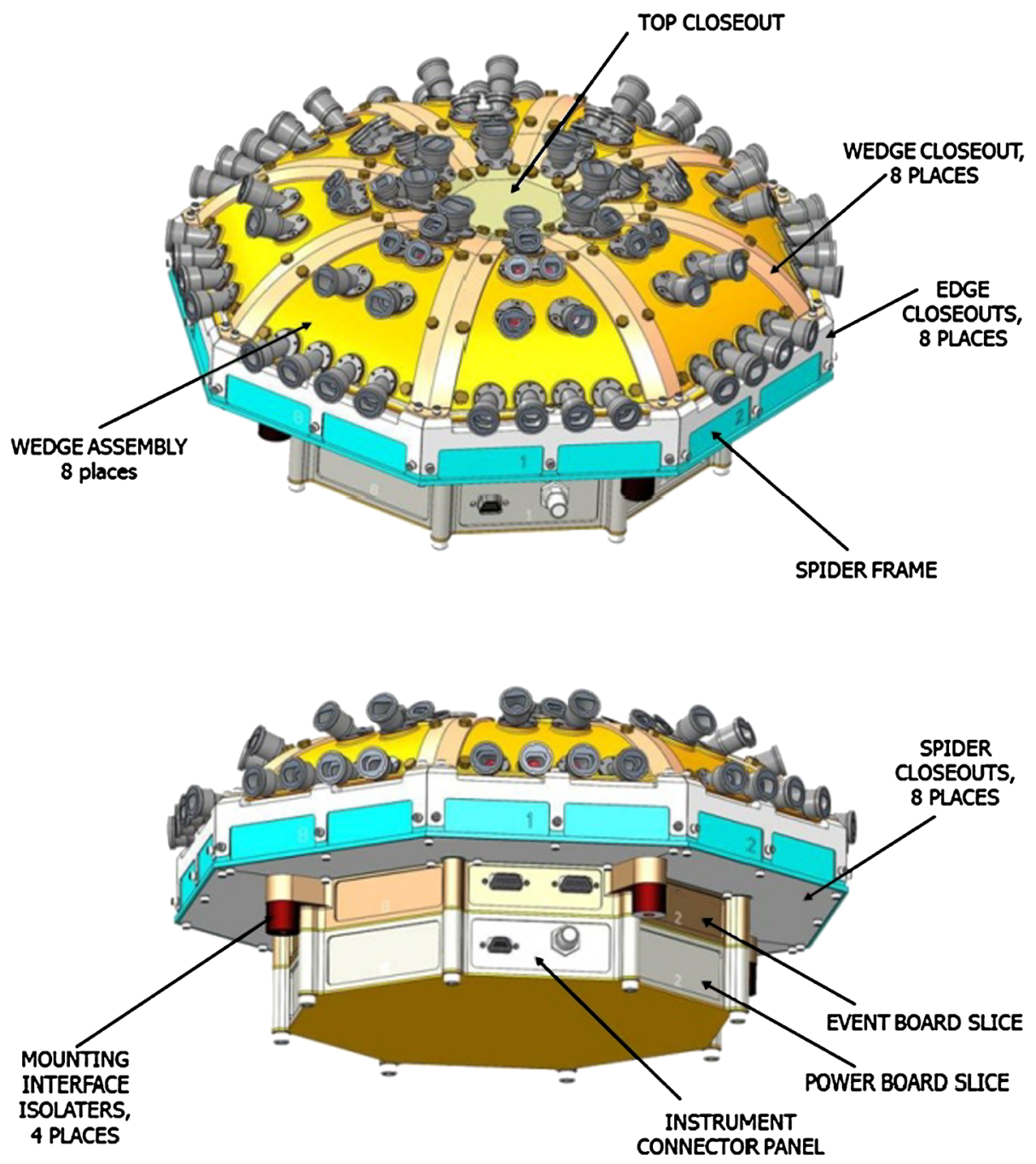

\section{TA008674}

Fig. 25 EPI-Lo mechanical assembly

mon software includes packet telemetry, command handling, macros (stored command sequences), memory management, monitoring and alarm generation, and status reporting. About $50 \%$ of the application software and $>90 \%$ of the instrument boot code is reused.

EPI-Lo software identifies ion species and energies on-board by referencing look up tables stored in non-volatile memory that define carefully tailored regions ("boxes") in the TOF vs. Energy parameter space. In the instrument's ion-composition mode, each incoming ion event is associated with a 32-bit species and energy accumulation bin that will record the total number of events per integration interval within the defined box. The accumulation bins are arranged in packets and sent to the SSR. A selection of raw event data is also sent to the SSR, allowing more detailed compositional studies (but with decreased statistics due to 

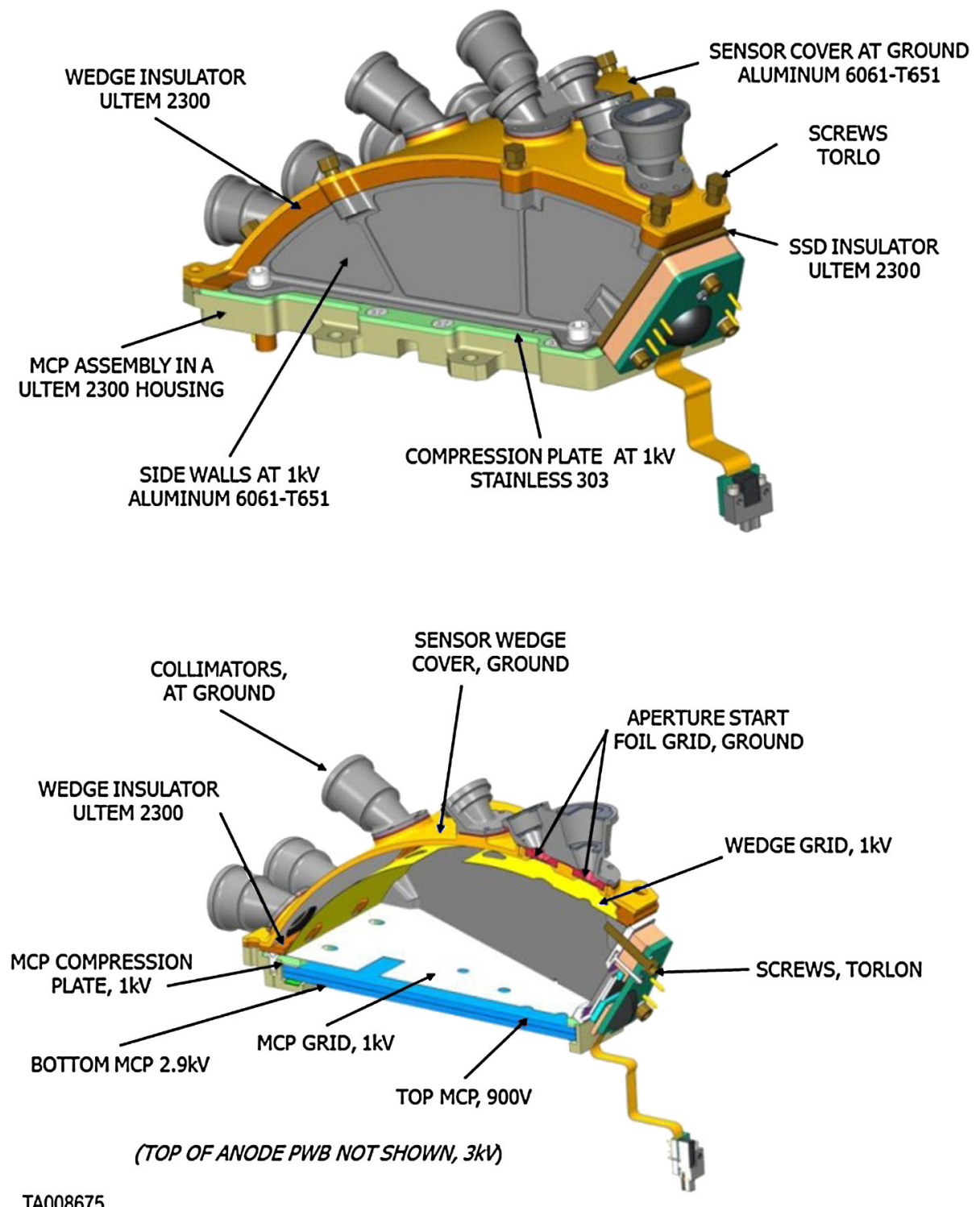

Fig. 26 Wedge design

downlink data volume constraints). We have used these techniques successfully many times on past missions (most recently, and with very similar software, on PEPSSI, RBSPICE, and JEDI).

In the spacecraft interface software, command and telemetry use the 115,200-baud Universal asynchronous receiver/transmitter (UART) protocol with 8 data bits plus odd parity. There is a redundant (side A and B) interface, for which the command arrival determines the active side; telemetry is sent only to that side. Dynamic side switching is supported. The 
system is interrupt driven: per-byte interrupt for command and telemetry, and side A and B 1PPS interrupts.

\subsection{Calibration Plan}

EPI-Lo measurements are intended to generate the information needed to derive differential intensity $j$ in $\left(\mathrm{cm}^{2} \mathrm{sr} \mathrm{s} \mathrm{keV/nuc}\right)^{-1}$. The goal of EPI-Lo characterization and calibration efforts is to develop the quantitative procedures for converting the count rates $R\left(\mathrm{~s}^{-1}\right)$ reported by EPI-Lo into estimates of $j$ for the various defined ranges of energies, particle species, and arrival angles.

Calibration for a particle instrument like EPI-Lo means determining the following:

1. Transfer function from counts into flux (engineering units to physical units)

2. Correct rates for effects such as dead times and hardware or software saturation

3. Response to visible and ultraviolet light

4. Response to high-energy (out of band) particle backgrounds

Calibration will be done primarily using the APL particle accelerator (e.g., McNutt et al. 2008), a versatile system capable of producing a broad range of ion species at energies from 20 to $170 \mathrm{keV}$. The system includes an electron-impact ionization source, extraction gap, Einzel Lens and Wien filter mounted in the insulated terminal structure along with all associated power supplies. The system produces beams of $\mathrm{H}, \mathrm{He}, \mathrm{O}$, and noble gas ions with intensities over the range of $10^{2}-10^{6}$ particles $\mathrm{cm}^{-2} \mathrm{~s}^{-1}$ at the target position $\left(\sim \mathrm{mm}^{2}\right.$ to $\mathrm{cm}^{2}$ ). These tests will be supplemented with a variety of radioactive sources (e.g., ${ }^{241} \mathrm{Am}$ ) as stimuli.

In addition, calibration runs will be made using the accelerator at Goddard Space Flight Center. A proton beam will be used to scan both angles to complete the characterization of the transfer function. The sensor response to electrons will be characterized from $\sim 100 \mathrm{keV}$ to $1 \mathrm{MeV}$, and heavy ions ( $\mathrm{He}, \mathrm{O}$, and $\mathrm{Ar}$ ) will also be used to characterize the instrument response. Calibrations of the response to both foreground and background ions and electrons at very high energies (up to $\sim 20 \mathrm{MeV}$ ) require additional specialized facilities. These include the accelerator at Lawrence Berkeley National Laboratory (Berkeley Lab) and an accelerator at Idaho National Laboratory (INL).

Calibration will begin on the prototype unit to validate the instrument design and performance. This activity will also be used to establish testing procedures for the flight unit. Most sensor-level calibration for EPI-Lo occurs sequentially, with subsystems sequenced through fixed calibration setups. Sensor wedges are integrated with the electronics, followed by instrument-level testing and pre-qualification calibration, which establishes a calibration baseline. The integrated EPI-Lo undergoes EMI/EMC, vibration, and thermal vacuum testing. Final calibration is performed to ensure no changes have occurred during environmental testing. In addition to ground calibration and pre- and post-environmental qualification, inflight calibration is used to finalize knowledge of the full instrument response. For instance, data will be used to monitor MCP gain, and efficiencies can be tracked separately for each wedge by using relative Start, Stop, and SSD rates. There are also on-board pulsers to calibrate the SSD and anode electronics. Such in-flight calibration times will also naturally allow cross-calibration between EPI-Lo and EPI-Hi.

\subsection{Radiation/Contamination Effects}

All EPI-Lo electronics parts have been used in recent flight instruments, are currently available, and have a minimum tolerance of $>100 \mathrm{krad}$. The electronics box thickness meets 
that dose level with a factor of two margin. EPI-Lo contains detectors sensitive to certain contaminants, e.g. hydrocarbons; however, all detectors reside within cavities behind cover foils protected pre-launch by GN2 purge. EPI-Lo is located out of direct impact by thruster plumes; high voltage can be turned down for thruster firings if analysis indicates temporary pressure increases from such events.

\subsection{Data Collection Strategy and Products}

EPI-Lo delivers telemetry in Consultative Committee for Space Data Systems (CCSDS) packets using the CCSDS file Delivery Protocol (CFDP). Most data are losslessly compressed (using the FAST algorithm) prior to packetization; compression factors of $>1.6$ have been achieved with similar instruments on recent deep-space missions (e.g., MESSENGER and New Horizons). Sensor-event collection, command-and-telemetry processing, and instrument control functions are implemented by the instrument processor, an approach successfully used for the previously mentioned instruments now in operation or development.

The telemetry formats are flexible and adjustable by ground command. EPI-Lo has low average data rates fitting the nominal allocation suggested in the original SPP Announcement of Opportunity (NASA Science Mission Directorate 2014) and high data rates to allow for higher time resolution and additional event data, sometimes called pulse height analysis (PHA) data. EPI-Lo has ion counting rates for up to 64 energy bins, at least 9 species, and 80 angular "pixels" (one for each aperture). Event data include high-resolution TOF and energy PHA values. Energy-spectra data are collected and averaged from the selected detectors. High-resolution electron rates are also collected.

Data products conform to time, angle, and energy-resolution requirements that address the science objectives. Rate data for ions are generated nominally at 30 and 5 s timeresolution (time resolution can also be adjusted by ground command between $1 \mathrm{~s}$ and several hours). High-resolution energy spectra are sent every few minutes so that fast developing features can be followed. The high level of flexibility enables maximal use of telemetry allocation and permits adjusting the operating parameters in response to evolving flight experience if needed.

\section{Energetic Particle Instrument-High Energy (EPI-Hi)}

EPI-Hi measures energetic particles in the upper portion of the ISIS energy range. As discussed in Sect. 2, the two ISIS instruments are complementary in their energy range and sky coverage in order to obtain the comprehensive set of observations needed to fully understand solar energetic particle sources, acceleration, and transport close to the Sun.

\subsection{EPI-Hi Overview}

EPI-Hi measures energetic electrons, protons, and heavy ions in the $\mathrm{MeV}$ energy range using the $d E / d x$ versus total energy technique in a sensor system based entirely on ionimplanted silicon SSDs. It builds on a heritage of more than 40 years of SSD-based energetic particle instruments, and most directly on the Low Energy Telescope (LET, Mewaldt et al. 2008b) and High Energy Telescope (HET, von Rosenvinge et al. 2008) that are part of the IMPACT instrument suite on the twin STEREO spacecraft; these instruments have been providing multi-point measurements of energetic particles in the heliosphere since late 2006. The sophistication of SSD instruments has steadily advanced as capabilities have improved 
Table 5 EPI-Hi instrument required and expected performance

\begin{tabular}{|c|c|c|c|}
\hline Parameter & Required & Goal (expected) & Comment/Heritage \\
\hline Electron energies & $0.5-3 \mathrm{MeV}$ & $0.5-6 \mathrm{MeV}$ & STEREO/HET \\
\hline Ion energies & $\sim 1$ to $\geq 50 \mathrm{MeV} / \mathrm{nuc}$ & $\begin{array}{l}p, \mathrm{He}: 1 \text { to } 100 \mathrm{MeV} / \mathrm{nuc} \\
Z \geq 6: 1.5 \text { to }>100 \mathrm{MeV} / \mathrm{nuc}\end{array}$ & STEREO/LET \& HET \\
\hline Energy binning & $\geq 6$ bins per decade & 12 bins/decade & Logarithmic bins \\
\hline Cadence & Fastest: $e 1 \mathrm{~s}, p 5 \mathrm{~s}$ & $\begin{array}{l}\text { Fastest: } e 1 \mathrm{~s}, p 1 \mathrm{~s} \\
\text { most data products: } 1 \mathrm{~min} \\
\text { angular distributions: } 5 \mathrm{~min}\end{array}$ & $\begin{array}{l}\text { Large energy bins best } \\
\text { energy resolution }\end{array}$ \\
\hline Fields of view & $\begin{array}{l}\geq \pi / 2 \text { steradians in } \\
\text { sunward and } \\
\text { anti-sunward } \\
\text { hemisphere }\end{array}$ & $\begin{array}{l}\text { Five } 45^{\circ} \text { half-angle view } \\
\text { cones covering sunward and } \\
\text { anti-sunward hemispheres }\end{array}$ & $\begin{array}{l}\text { View cones overlap to } \\
\text { provide full energy } \\
\text { coverage near Parker } \\
\text { spiral to within } 10^{\circ} \text { of } \\
\text { spacecraft-Sun line }\end{array}$ \\
\hline $\begin{array}{l}\text { Angular } \\
\text { sectoring }\end{array}$ & $e: \leq 45^{\circ} ;$ ions: $\leq 30^{\circ}$ & $\begin{array}{l}45^{\circ} \text { half-angle cones } \\
\text { subdivided into } 25 \\
\text { overlapping sectors }\end{array}$ & $\begin{array}{l}\text { Overlapping sectors } \\
\text { provide improved angular } \\
\text { resolution for deriving } \\
\text { pitch angle distributions }\end{array}$ \\
\hline $\begin{array}{l}\text { Elemental } \\
\text { composition }\end{array}$ & $\begin{array}{l}\mathrm{H}, \mathrm{He}, \mathrm{C}, \mathrm{O}, \mathrm{Ne}, \mathrm{Mg} \\
\mathrm{Si}, \mathrm{Fe}\end{array}$ & $\begin{array}{l}\mathrm{H}, \mathrm{He}, \mathrm{C}, \mathrm{N}, \mathrm{O}, \mathrm{Ne}, \mathrm{Na}, \mathrm{Mg} \\
\mathrm{Al}, \mathrm{Si}, \mathrm{S}, \mathrm{Ar}, \mathrm{Ca}, \mathrm{Cr}, \mathrm{Fe}, \mathrm{Ni}\end{array}$ & STEREO/LET \\
\hline $\begin{array}{l}\text { Isotopic } \\
\text { composition }\end{array}$ & ${ }^{3} \mathrm{He} /{ }^{4} \mathrm{He}$ & ${ }^{3} \mathrm{He} /{ }^{4} \mathrm{He}$ & $\begin{array}{l}\text { In selected viewing } \\
\text { directions STEREO/LET }\end{array}$ \\
\hline Intensity range & $\begin{array}{l}\text { Up to } 3 \times 10^{6} \text { protons } \\
\mathrm{cm}^{-2} \mathrm{sr}^{-1} \mathrm{~s}^{-1}\end{array}$ & $\begin{array}{l}\text { Normal mode: up to } \\
\sim 1 \times 10^{6} \text { protons } \\
\mathrm{cm}^{-2} \mathrm{sr}^{-1} \mathrm{~s}^{-1} \\
\text { Pixel mode: up to } \sim 4 \times 10^{7} \\
\text { protons } \mathrm{cm}^{-2} \mathrm{sr}^{-1} \mathrm{~s}^{-1}\end{array}$ & $>10 \mathrm{MeV}$ protons \\
\hline $\begin{array}{l}\text { Geometrical } \\
\text { factor }\end{array}$ & N/A & $\begin{array}{l}5 \text { view cones, each with } \\
A \Omega \approx 0.5 \mathrm{~cm}^{2} \mathrm{sr}\end{array}$ & $\begin{array}{l}\text { Value at energy with } \\
\text { maximum } A \Omega\end{array}$ \\
\hline
\end{tabular}

with high performance, low power, and miniaturized, front-end electronics in the form of ASICs. The EPI-Hi requirements and expected performance are summarized in Table 5.

Figure 27 shows a block diagram of EPI-Hi. The sensor system consists of three detector stacks, commonly called "particle telescopes", each controlled by a dedicated electronics board that provides front-end analog signal processing, PHA, coincidence determination, data accumulation and analysis, and formatting for readout. We have tailored the telescope designs for measurements in two different but overlapping energy ranges. A pair of Low Energy Telescopes (LET1 and LET2) measure protons and heavy ions from $\sim 1$ to $>20 \mathrm{MeV} /$ nuc and electrons from $\sim 0.5$ to $\sim 2 \mathrm{MeV}$ while a single High Energy Telescope (HET) extends the energy coverage for electrons up to $\sim 6 \mathrm{MeV}$ and for protons and heavy ions to at least $100 \mathrm{MeV} /$ nuc. LET1 and HET analyze particles incident from both ends of their detector stacks while LET2 analyzes particles incident from only one end because the other end is blocked by the spacecraft. Each of the five active telescope ends has an angular acceptance cone with a half-angle of $45^{\circ}$ centered on the symmetry axis of the telescope.

In addition to the three telescope boards, EPI-Hi includes a Data Processing Unit (DPU) board that is responsible for the overall control of the instrument, including managing the boot-up of processors on the telescope boards, collecting data from those boards and merging them into a single data stream that is passed to the spacecraft, and receiving commands 


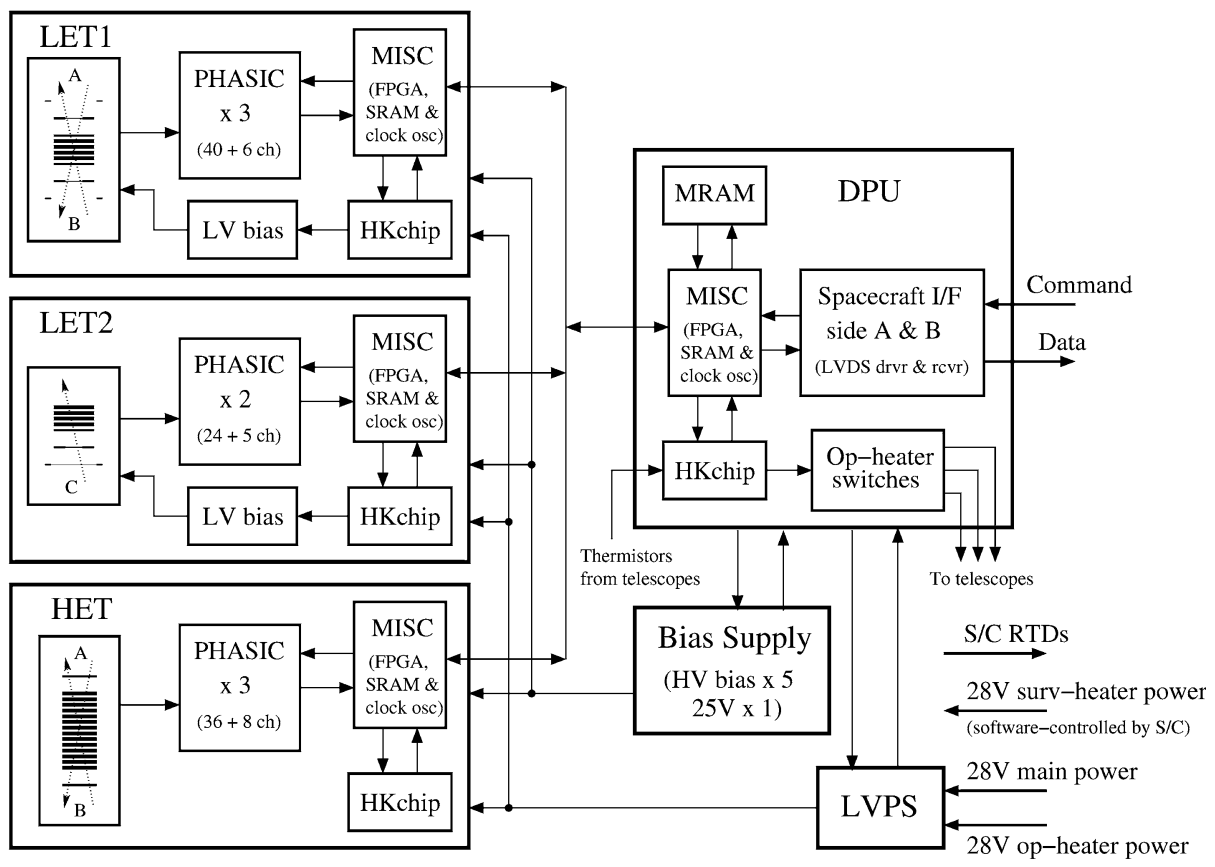

Fig. 27 EPI-Hi block diagram

from the spacecraft and passing them to the processor for which they are intended. The remaining two electronics boards contain the LVPS that produces the required regulated, filtered voltages needed by the instrument and the bias supply that produces programmable voltages for biasing SSDs.

A metal enclosure, designated the "E-Box", holds the electronics boards and provides a degree of shielding against radiation and high-speed dust impacts. We also use the E-Box, which is attached to the ISIS bracket (see Sect. 2.3), as a platform on which we mount the detector telescopes in the orientations needed to achieve the required viewing directions, as illustrated in Fig. 28.

\subsection{High-Energy Telescope}

Figure 29 shows cut-away views of the telescopes. HET has a thick central stack of detectors together with a single detector spaced farther away at each end. A coincidence between this front detector and the central stack defines the FOV of the telescope. The front detectors (H1) and the outermost detectors at each end of the central stack (H2) have five main active elements, consisting of a central bull's-eye and four quadrants of a surrounding annular region, with all elements having equal areas, as illustrated in Fig. 30a. Identifying which detector segment was hit by an incident particle in each of the two detectors allows us to subdivide the FOV into 25 overlapping sectors, thus enabling measurements of the distribution of particle incidence directions.

The detectors other than $\mathrm{H} 2$ in the central HET stack have a central segment with an active area equivalent to the combined areas of the five segments in $\mathrm{H} 1$ and $\mathrm{H} 2$. The combination of the thickness of the central stack and the active diameter of the detectors is such that 
Fig. 28 EPI-Hi mechanical configuration including E-Box and telescopes
LET1

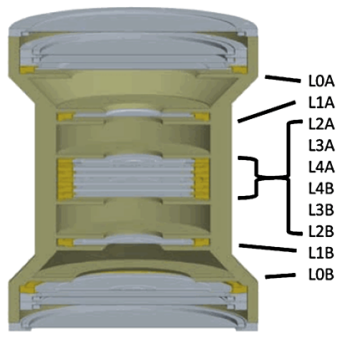

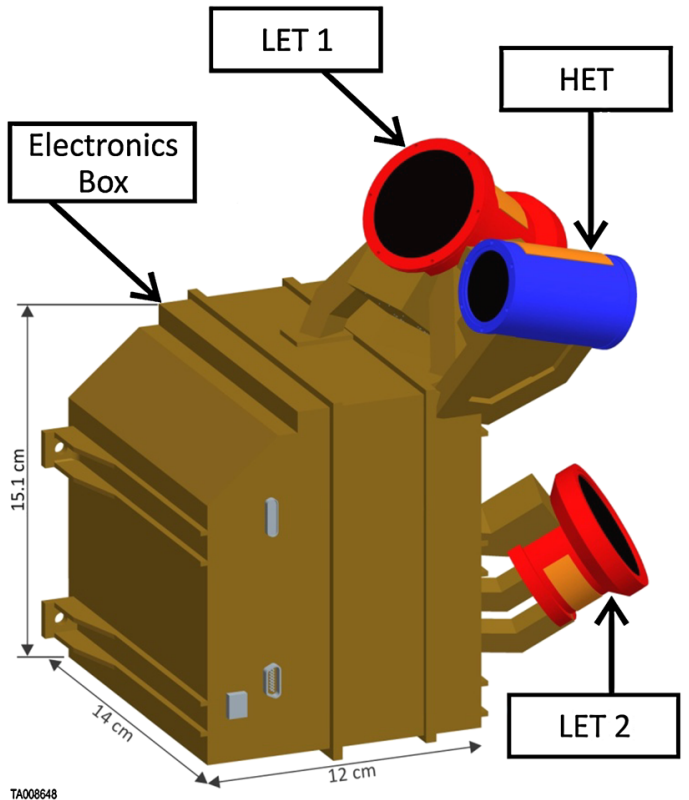

TA003548

LET2

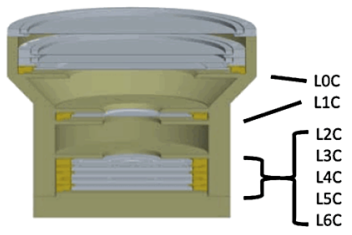

HET

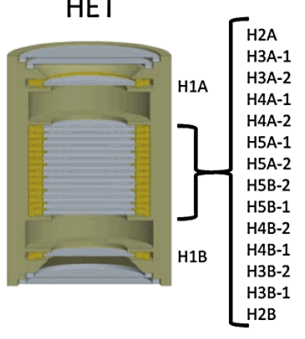

Fig. 29 Cut-away illustration of the EPI-Hi telescope designs and detector naming

some particles incident at large angles from the telescope axis will exit through the sides of the stack. To identify and reject these side-exiting particles, the detectors include an annular "guard" segment that is used as anti-coincidence in the analysis. The guard also allows identification of particle trajectories that clip the detector edge resulting in reduced energy-loss signals. This feature is important because the detector thickness is a non-negligible fraction of the detector diameter and thus edge-clipping trajectories are not improbable. The HET detectors (as well as the LET detectors other than L0 and L1) also include one additional segment in the form of a small pixel that is used to extend the dynamic range in particle intensities to values above which the larger detector segments will saturate, as discussed in Sect. 4.5.

The individual silicon detectors used in the HET telescopes have thicknesses of either $500 \mu \mathrm{m}(\mathrm{H} 1)$ or $1000 \mu \mathrm{m}$. However, for H3, H4, and H5, we use two successive $1000 \mu \mathrm{m}$ detectors connected to the same front-end electronics to produce what is effectively a $2000 \mu \mathrm{m}$ detector without requiring the high bias voltage that an individual detector of this thickness would need. 




Fig. 30 (a) Illustration of the detector segmentation. Detectors L0 through L3 are used in LET; detectors H1 through $\mathrm{H} 3$ are used in HET. The dashed line in the L0 detector drawing indicates the diameter inside which the silicon thickness is $12 \mu \mathrm{m}$. The L4, L5, and L6 detectors are identical to L3 while the H4 and H5 detectors are identical to H3. (b) Photograph of a prototype L1 detector

\subsection{Low-Energy Telescope}

The conceptual design of the LETs is similar to that of HET, using a central detector stack in conjunction with position-sensitive detectors that define the FOV and subdivide it into a number of sectors that we use for measuring particle angular distributions. Similar to HET, the LET detectors, other than L0 and L1, have thicknesses of either $500 \mu \mathrm{m}$ (L2) or $1000 \mu \mathrm{m}$. In order to achieve a low threshold energy for EPI-Hi and to minimize the energy gap between EPI-Hi and EPI-Lo, we have developed a process for fabricating very thin silicon detectors. At the front of LET we have L0 and L1 detectors with thicknesses of $12 \mu \mathrm{m}$ and $25 \mu \mathrm{m}$, respectively (Fig. 29). A $1 \mathrm{MeV}$ proton has energy just sufficient to penetrate the L0 detector and the thin windows in front of the telescope $(\sim 3 \mu \mathrm{m}$ silicon equivalent) and provide the 2-parameter measurement required for particle identification. The thin detectors are sufficiently uniform in thickness to allow the required species separation, including distinguishing between the isotopes of $\mathrm{He}$ as shown in Sect. 4.4. Like the $\mathrm{H} 1$ and $\mathrm{H} 2$ detectors, L0, L1, and L2 are all position sensitive with a central bull's-eye surrounded by an annulus subdivided into four quadrants. Figure 30b shows a photograph of a prototype L1 detector.

Protons with energy greater than $\sim 8 \mathrm{MeV}$ will have energy losses in the $\mathrm{L} 0$ detector that fall below the detection threshold of the front-end electronics (see Sect. 4.7). For this reason, in LET we identify events either by a coincidence between L0 and L1 or a coincidence between L1 and L2. It is possible for an event to satisfy the L1•L2 coincidence without triggering L0 either because the signal fell below threshold in L0 or because the particle trajectory did not pass through the active area of this device. We have designed the L0 detector to have its $1 \mathrm{~cm}^{2}$ active area located at the center of a large $\left(\sim 9 \mathrm{~cm}^{2}\right)$ thin silicon membrane that covers the entire field of view defined by the L1•L2 coincidence. This ensures that the analyzed particles will have passed through a consistent amount of material, thereby enabling accurate calculations of their incident energies. The L2 detector has a large annular guard region so that we can determine whether an event defined by a coincidence between 
Fig. 31 Species and energy coverage by LET and HET telescopes

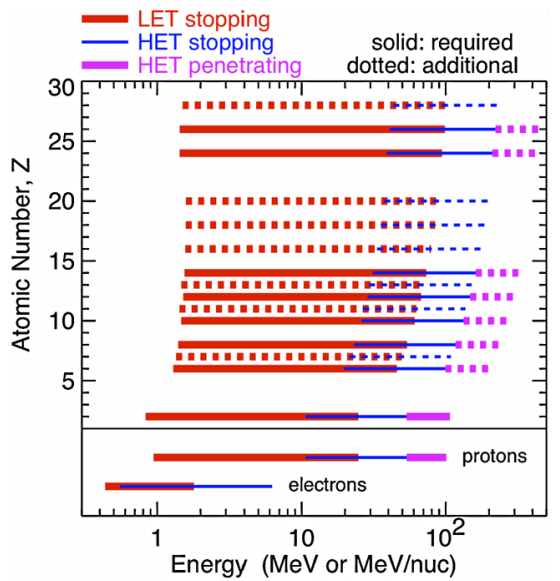

L0 and the center element of L1 but lacking a signal in the central five segments of L2 is due to a particle stopping in L1 or to a trajectory passing outside the central part of L2.

Unlike in HET, the central stack detectors in LET (L3 and L4) have an active diameter large enough to intercept even the widest-angle trajectories defined by the L1•L2 coincidence. Thus, the LET detectors do not require guard segments to identify side-exiting particles. We have also segmented these detectors into a central bull's-eye surrounded by a wide annular region so that we can dynamically adjust the LET geometrical factor and thereby increase the dynamic range in particle intensities that EPI-Hi can measure (see Sect. 4.6).

\subsection{Species and Energy Coverage}

Figure 31 shows the energy range covered by LET and HET for elements with atomic numbers in the range $1 \leq Z \leq 30$. The primary measurements involve the analysis of particles that trigger two position-sensitive detectors and then stop in the central detector stack, as indicated by the absence of a signal in the detector at the opposite end of the stack. In the case of HET we extend the coverage to higher energies by also analyzing events due to particles that penetrate the entire thickness of the stack.

Figure 32 shows results from a Monte Carlo simulation of the HET response to a population of particles with heavy-ion composition typical of a large SEP event. The element response tracks have distinct branches that correspond to stopping and penetrating particles. For events on the stopping branch we derive the particle energy by simply adding the energy losses in all of the detectors and making a small correction for energy lost in the front windows. On the penetrating branch the detected energy is less than the incident energy, but we are able to use the location along the track to obtain a reasonably accurate value for the incident energy up to about twice the energy required to penetrate the stack. This allows us to measure energy spectra up to at least $100 \mathrm{MeV} /$ nuc for the more abundant elements.

Figure 33 shows results from a Monte Carlo simulation of the LET response to particles that satisfy the L1•L2 coincidence and stop in L2. The figure shows L1 and L2 energy losses with a simple multiplicative correction that depends on the mean secant of the incidence angle in the assigned sector (Mewaldt et al. 2008b). This correction, which removes a significant fraction of the track broadening caused by variations in the angle at which the particles penetrate the L1 detector, allows us to display all of the events in well resolved tracks on a single plot. 
Fig. 32 Monte Carlo simulation of HET response measuring $\Delta E$ using the front detector (H1A) and the residual energy, $E^{\prime}$, using all of the detectors in the central stack. Relative abundances are typical of a large SEP event except that He has been suppressed by a factor of 200 and $\mathrm{H}$ by 2000
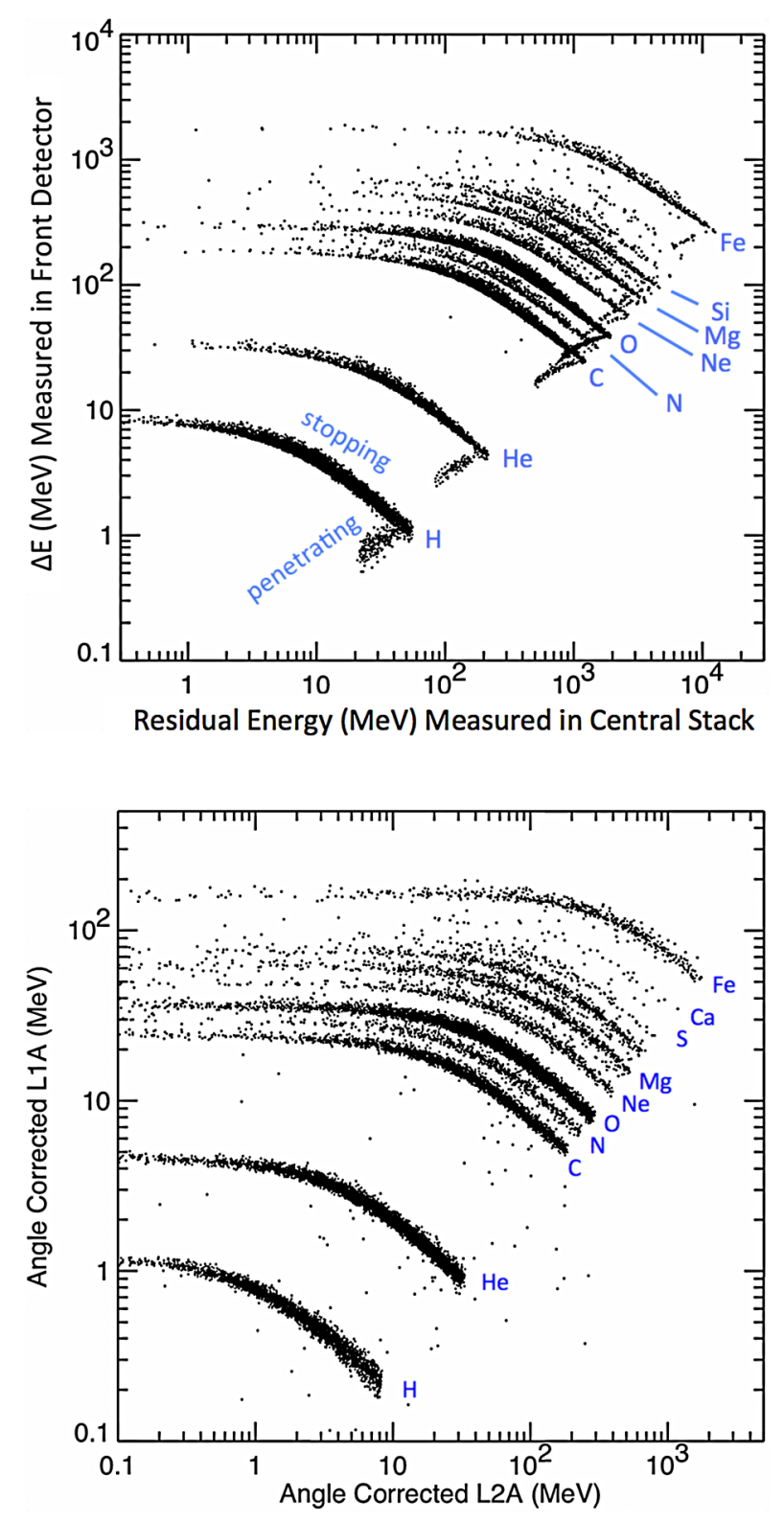

Fig. 33 Monte Carlo simulation of the LET response for particles stopping in the L2A detector. A simple multiplicative correction factor depending on the angular sector in which each particle was detected has been applied to the $\Delta E(\mathrm{~L} 1 \mathrm{~A})$ and $E^{\prime}$ (L2A) values in order map all of the events onto a common response matrix

In addition to separating the response tracks for major elements, we have designed the EPI-Hi telescopes to distinguish helium isotopes. A large enhancement of ${ }^{3} \mathrm{He}$ relative to ${ }^{4} \mathrm{He}$, sometimes by factors of $10^{3}$ or greater relative to the solar coronal value of $\sim 4 \times 10^{-4}$, is a distinguishing characteristic of impulsive SEP events in which particle acceleration is thought to be associated with magnetic reconnection rather than due to coronal and interplanetary shocks (see Sect. 1). For He isotope measurements we use portions of the FOV that have small angles, $\theta$, relative to the telescope axis, thus minimizing the uncertainty in the $d E / d x$ measurement due to the range of particle path-lengths through the $d E / d x$ 
Fig. 34 Measured response of 2-detector telescope consisting of one L0 detector and one L1 detector to ${ }^{4} \mathrm{He}$ particles from a ${ }^{244} \mathrm{Cm}$ source. A piece of fine fabric was interspersed between the source and the detectors to produce a spread of energies. The inset shows a mass histogram accumulated over the residual energy interval from 1.0-2.5 MeV. Red curves are approximate tracks for ${ }^{3} \mathrm{He}$ and

${ }^{4} \mathrm{He}$ calculated from a simple range-energy relation

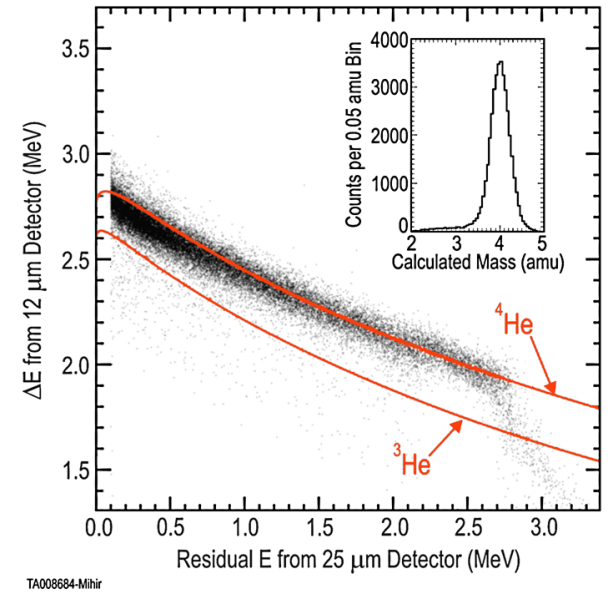

TA008684-Mihir

detector ( $\sec \theta$ variation). The separation of He isotopes is most challenging at the lowest energies that we measure with LET since the effects of detector thickness non-uniformities and of Bohr/Landau fluctuations (e.g., Ahlen 1980) in the particle energy losses are most significant there. Because ${ }^{4} \mathrm{He}$ in this energy range can be obtained from radioactive alphaparticle sources, we were able to test the performance in the laboratory using an L0 detector to measure $d E / d x$ and an L1 detector to measure residual energy. Figure 34 shows the ${ }^{4} \mathrm{He}$ response track obtained from these measurements and compares it with track locations calculated from a simple parameterization of the range-energy relation in silicon. As shown in the inset, the ${ }^{4} \mathrm{He}$ resolution is sufficient to distinguish ${ }^{3} \mathrm{He}$ from ${ }^{4} \mathrm{He}$ down to ratios as small as a few percent.

The energy range for electron measurements in HET extends from the energy required to penetrate the $\mathrm{H} 1$ detector and front windows up to a maximum set by the active thickness of the entire HET detector stack. In LET, electron energy losses in the L0 and L1 detectors always fall below threshold so we use coincidences among L2 and the following stack detectors for electron detection. In this case we do not have trajectory information (other than the telescope end that the electron entered), so we use a model of the instrument response to derive electron energy spectra from the data. Data from the LETs are important for obtaining electron energy spectra measurements over a broad field of view as well as for determining electron event onset times in cases where the first-arriving electrons do not fall within the HET field of view. The EPI-Hi fields-of-view provide excellent coverage close the Parker spiral direction over the full orbit and typically will allow measurements of the first-arriving particles.

\subsection{Viewing Directions and Angular Sectioning}

Figure 18 shows the locations of the EPI-Hi $45^{\circ}$ half-angle conical FOVs in the sky. Although these fields are largely unobstructed, there are several places where portions of the spacecraft or of other instruments block them. The most significant blockage is by the spacecraft's TPS. The forward view cones of HET and LET1 are intentionally oriented so that the TPS cuts through them, thus allowing detection of particles arriving from as close as possible to the radial direction over the full EPI-Hi energy range. The combination of the EPI-Hi telescopes will allow measurement of nearly complete pitch angle distributions for nominal 


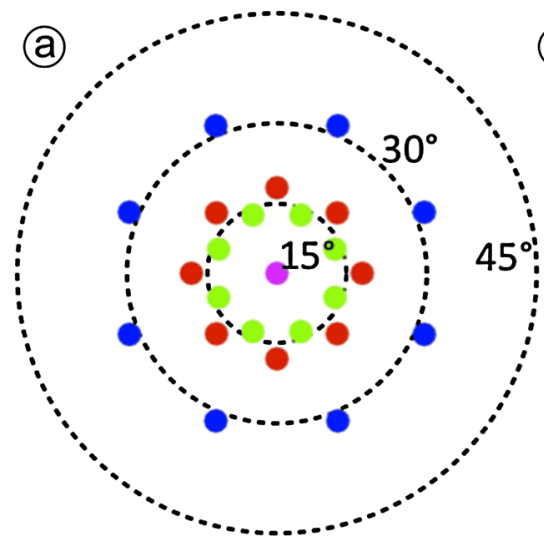

TA008673

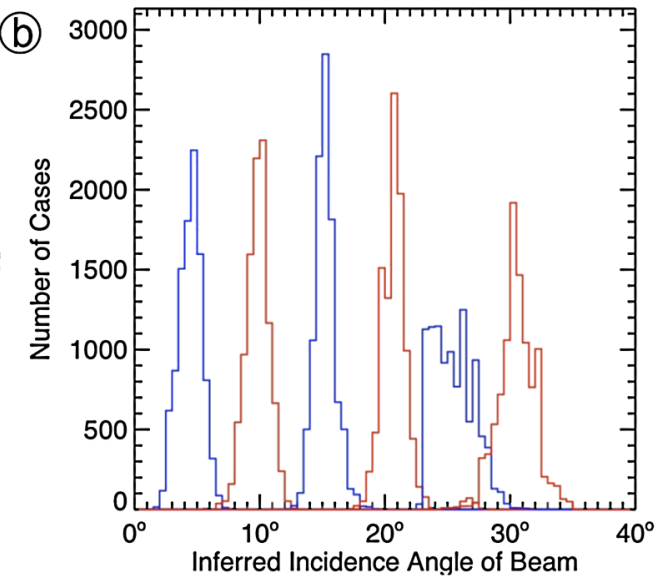

Fig. 35 (a) Mean viewing directions of each of the 25 sectors. Dashed circles indicate angles of $15^{\circ}, 30^{\circ}$, and $45^{\circ}$ from the telescope axis. (b) Simulted distributions of derived incidence directions for parallel beams incident at $5^{\circ}, 10^{\circ}, 15^{\circ}, 20^{\circ}, 25^{\circ}$, and $30^{\circ}$ from the telescope axis when determining the direction using 200 detected particles

Parker spiral magnetic fields over the entire orbit except for blockage by the TPS, which affects less than $10^{\circ}$ even near perihelion. A number of the EPI-Lo apertures (Fig. 19) are also oriented for viewing within the overlap region between the HET and LET1 forward fields of view, thus allowing excellent spectral coverage in the region where the average Parker spiral magnetic field will often be located when the spacecraft is near the Sun.

We assign detected particles to one of 25 sectors based on which of the 5 main detector segments they hit in two position-sensitive detectors (Fig. 30). By orienting one of the two detectors with its quadrant sectors rotated $45^{\circ}$ relative to those on the other detector we are able to identify 25 unique mean viewing directions, as illustrated in Fig. 35a. The angular width of the individual sectors is broad, extending as much as $25^{\circ}$ from the mean direction in some cases. However, $\geq 80 \%$ of the geometrical acceptance of the sectors fall within $15^{\circ}$ of the mean. We rely on the significant overlaps among the sectors to provide information about particle angular distributions, as illustrated in Fig. 35b, where we show the accuracy with which the arrival direction of a narrow beam of particles can be determined as a function of that direction and of the number of particles counted.

\subsection{Geometrical Factors and Dynamic Range in Particle Intensities}

We have designed EPI-Hi to measure MeV particles over a large dynamic range of intensities, since this range must encompass the combined effects of varying numbers of accelerated particles, varying radial distances from the Sun, and varying magnetic connection to the acceleration region, as well as intrinsic differences in the source abundances among different species. To accommodate this large range, the telescopes have relatively large geometrical factors to cover the lowest intensities of interest but at the same time use detectors that are small and closely spaced to minimize the geometrical factor for out-of-geometry particles that can cause backgrounds and dead time. We further reduce the effect of these unwanted particles by using a relatively thick housing for the telescopes that serves as a passive shield to absorb low energy particles. In addition, by using segmented detectors we are able to 
Fig. 36 (a) LET and HET geometrical factors as a function of the particle range in silicon. Energy scales for $\mathrm{H}, \mathrm{O}$, and $\mathrm{Fe}$ are also shown. Blue curves are used for LET, red curves for HET. Protons above $\sim 25 \mathrm{MeV}$ have energy losses in the L1 detector that fall below threshold and thus do not produce a coincidence in LET. Geometrical factors are shown for one end of each telescope. Plot is based on stopping particles; penetrating particle analysis will allow modest extensions to higher energies. (b) Solid (dashed) curves show the angles inside which $90 \%(50 \%)$ of the trajectories from an isotropic particle distribution fall as a function of the particle range in silicon

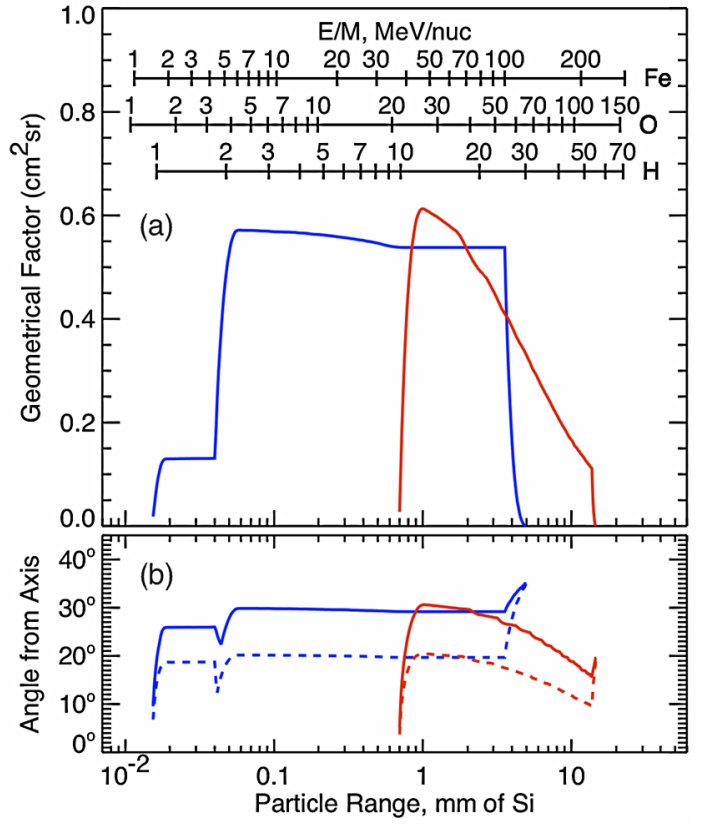

reduce the geometrical acceptance when particle intensities are high and thereby help avoid saturating the front-end electronics.

Figures 36a and 36b show the dependence of the geometrical factors of the HET and LET telescopes on incident particle energy and on particle arrival direction relative to the telescope axis, respectively. We calculated these geometrical factors for a single LET or HET entrance aperture and have not included corrections for blockages of the fields of view or for the geometrical factor reductions that we impose when intensities are high.

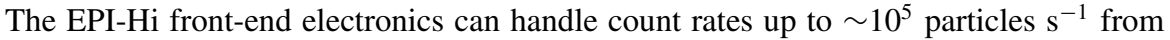
a single telescope before the analysis dead time approaches $100 \%$ (see Sect. 4.7). In order to keep the dead-time percentage significantly below this value, we employ a system of "dynamic thresholds" based on an approach that we successfully used in the STEREO/LET (Mewaldt et al. 2008b) and HET (von Rosenvinge et al. 2008) instruments. This approach takes advantage of the fact that the count rates are typically dominated by protons, electrons, and He nuclei, which have relatively small energy losses in the detectors. When the intensity gets high enough to cause excessive dead time, the thresholds are raised automatically on some segments in a detector so that they will no longer be sensitive to these low pulse heights but will still remain sensitive to heavy ions. By raising thresholds in this way for successively deeper detectors in the stack we are able to reduce the geometrical factor for electrons, protons, and $\mathrm{He}$ in several stages. We base the determination of when thresholds need to be raised (and when they subsequently need to be lowered as intensities decrease) on the count rate in a detector segment for which we do not raise the threshold. We take the threshold changes into account when we derive particle intensities from the measured event rates. Based on historical data and the assumption that SEP intensities will scale as $1 / r^{3}$ (an extreme assumption), we expect only one event during the prime mission that will have a peak intensity too high to measure with the HET telescope using the dynamic thresholds (Lario and Decker 2011). 
Estimates of the proton intensities that could be encountered if a large solar energetic particle event occurs when the spacecraft is close to perihelion and well connected to the acceleration region are extreme (Lario and Decker 2011), with a $5 \%$ chance that we could experience an event with a proton integral intensity above $1 \mathrm{MeV}$ exceeding $1.5 \times 10^{8} \mathrm{~cm}^{-2} \mathrm{sr}^{-1} \mathrm{~s}^{-1}$. If Solar Probe Plus should experience a rare event approaching this intensity, the count rate in even one of the detector segments will be high enough to saturate the front-end circuitry. In order to obtain a rough measure of intensity under such circumstances we have designed all of the thick detectors to include an extra small "pixel" segment with an area of $1 \mathrm{~mm}^{2}$ (1000 $\mu \mathrm{m}$ detectors) or $0.36 \mathrm{~mm}^{2}$ (500 $\mu \mathrm{m}$ detectors), which is $\leq 5 \%$ of the area of the smallest normal segment (see Fig. 30a). We use pixels on detectors at a few depths in the telescopes to measure singles count rates of energy losses exceeding a selected threshold. By setting the threshold slightly below the energy that will be deposited by a proton stopping in the full thickness of the detector $(\sim 12 \mathrm{MeV}$ for $1000 \mu \mathrm{m}$ detectors and $\sim 8 \mathrm{MeV}$ for $500 \mu \mathrm{m}$ detectors we can use the measured count rate to infer an approximate proton integral intensity above an energy that is set by the material above the pixel. Using a simulation of the instrument response we can combine measurements from pixels located at a few different depths to derive a rough energy spectrum for extreme events. From the correlation between intensity measurements made using the pixels and those obtained in the normal operating mode of the instrument we are able to calibrate the pixel measurements using data that we obtain before and after the highest intensity periods when normal operation is not possible. Referring to Fig. 13, we estimate that in the LET telescopes the pixels will be used for measurements when the integral intensity of protons above $10 \mathrm{MeV}$ exceeds $\sim 2 \times 10^{4} \mathrm{~cm}^{-2} \mathrm{sr}^{-1} \mathrm{~s}^{-1}$ and in HET when the intensity exceeds $\sim 10^{6} \mathrm{~cm}^{-2} \mathrm{sr}^{-1} \mathrm{~s}^{-1}$.

\subsection{Front-End Electronics}

The telescope boards process the signals from the silicon detectors using an ASIC called the "Pulse Height Analysis System Integrated Circuit" (PHASIC). A first generation version of this part is flying in the STEREO/LET and HET instruments. Solar Probe Plus requires a higher radiation tolerance than STEREO, so we have made several modifications to the PHASIC design to facilitate the production of a version that can tolerate a dose of at least $100 \mathrm{krad}$ and also to improve several key aspects of its performance.

The PHASIC contains 16 complete dual-gain PHA chains. Figure 37 shows a schematic diagram of one of these chains. It consists of a charge-sensitive preamplifier that drives two independent post-amplifiers that shape the pulse and provide additional gain. By having two parallel analysis chains we are able to make precise pulse height measurements over the preamplifier's entire dynamic range. The shaped pulse, which is bipolar with a peaking time of $1.9 \mu \mathrm{s}$, drives a peak detector that contains a linear gate and discriminator. The discriminator controls the linear gate and prevents subsequent pulses from contaminating the detected peak level until it has been digitized. The PHASIC uses Wilkinson rundown ADCs, which determine the pulse height by measuring the time interval required for discharging a hold capacitor using a constant current. The PHASIC's on-chip digital circuitry also counts the number of triggers of the high- and low-gain analysis chains and provides several programmable-input OR-gates that are used to perform the lowest level of event coincidence logic and thereby enable rapid rejection of some uninteresting triggers without requiring readout of the digitized pulse heights. The PHASIC developed for EPI-Hi also incorporates shift registers to record the time-history of the discriminator outputs and aid in identification of crosstalk. Each dual-gain PHA includes a precision test pulser for use in the functional testing and calibration of the circuit. 


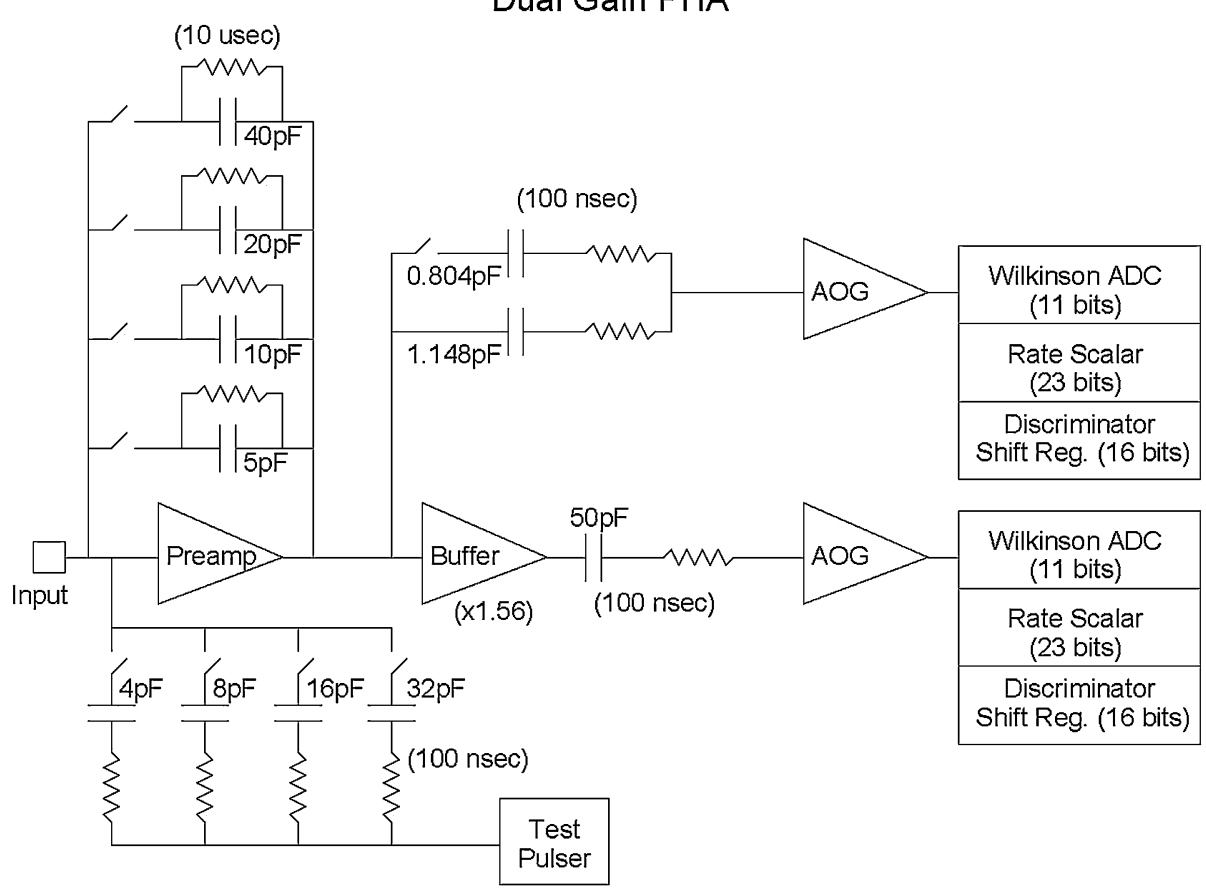

Fig. 37 Schematic diagram of one PHASIC channel

The PHASIC design includes several parameters that are programmable by setting bits in a digital control register. This programmability provides sufficient flexibility so that the PHASIC can meet the analysis requirements of all of the different detectors used in EPI-Hi. By programming the feedback capacitance in the preamplifier we select the gain appropriate to the range of signals for a given thickness of detector. We can also select between two options for the gain ratio between the high- and low-gain analysis chains. Other programmable parameters include the discriminator thresholds and the value of the capacitor used by the test pulser for injecting a calibrated charge into the preamplifier.

Table 6 summarizes the PHASIC specifications. As illustrated in Fig. 38, we install the ASIC in a hybrid circuit containing a number of passive components that support the operation of the chip. These include a precision resistor for each PHA chain that sets the ADC's rundown current and provides excellent stability over temperature. The design of the ASIC itself relies on ratios of component values for setting key parameters, since such ratios are much more predictable and stable than absolute component values.

We have fabricated and tested a prototype version of the EPI-Hi PHASIC using the commercial ON-Semi C5N CMOS process that will allow the addition of Aeroflex processing steps to improve the total tolerance to $>100 \mathrm{krad}$.

\subsection{Digital Control and Data Processing}

We use individual "Minimal Instruction Set Computers" (MISCs) to control each of the three telescope boards and also the DPU board. The STEREO and NuSTAR missions previously used the same MISC design, which is implemented in a portion of an FPGA and runs a Forth 
Table 6 PHASIC chip specifications

Number of dual-gain PHAs

Chip size

Power

Dynamic range

Integral non-linearity

Differential non-linearity

High/Low gain ratio

ADC type

ADC resolution (each gain)

Shaping

Preamp feedback capacitance

Preamp full scale output swing

Cross-talk between PHAs

Radiation tolerance

Gain temperature coefficient

Offset temperature coefficient

Operating temperature range

Leakage current balancing

Threshold programmability

Deadtime per trigger

\section{6}

7.4 by $7.4 \mathrm{~mm}$

$11 \mathrm{~mW}$ per active PHA

Up to 23000 (full scale/trigger threshold)

$<0.05 \%$ of full scale

$<1 \%$

68 or 40 , configurable

Wilkinson

11 bits, 12th bit overflow

Bipolar, $1.9 \mu \mathrm{sec}$ to peak

5-75 pF, programmable in $5 \mathrm{pF}$ steps

4.0 Volts

$<0.2 \%$

$>100 \mathrm{krad}$, no latchup below $80 \mathrm{MeV} /\left(\mathrm{mg} / \mathrm{cm}^{2}\right)$

$<50 \mathrm{ppm} /{ }^{\circ} \mathrm{C}$

$<0.1$ channel $/{ }^{\circ} \mathrm{C}$

-30 to $+50{ }^{\circ} \mathrm{C}$

Up to $32 \mu \mathrm{A}$ with 10-bit resolution

Up to $6 \%$ of full scale (each gain) with 10 bit resolution 4-67 $\mu$ s (pulse height dependent)

Fig. 38 Photograph of PHASIC chip installed in hybrid. Centimeter scale at right indicates size

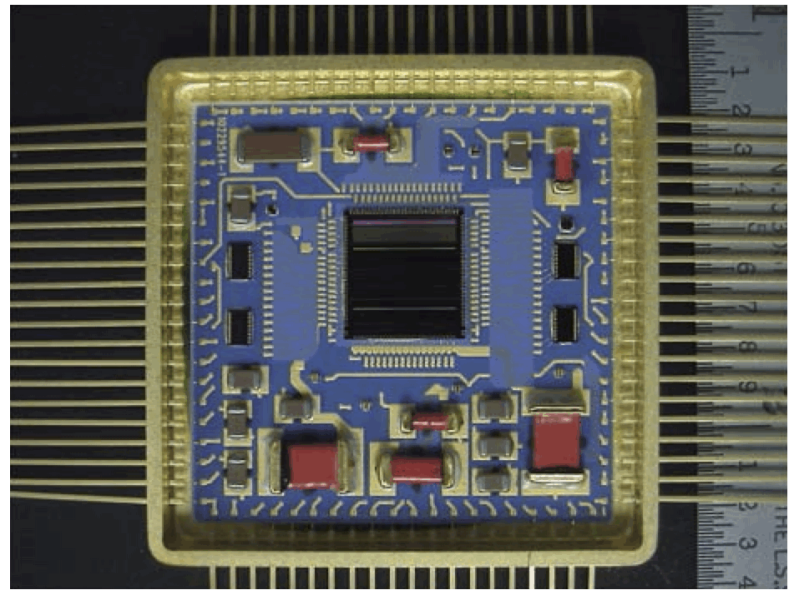

operating system (Mewaldt et al. 2008b). The logic circuitry required by each of the boards also fits in the RTAX250 FPGA, with significant margin. Each MISC has an associated SRAM chip to provide read-write memory and, in addition, the DPU MISC has non-volatile magnetoresistive random-access memory (MRAM) that contains the code and tables needed by all of the MISCs. Working from this stored copy of the software, the DPU MISC supports the booting of the MISCs on the telescope boards ("telescope MISCs") when needed.

The telescope MISCs handle the sorting of events that satisfy the coincidence requirements implemented in the PHASIC and FPGA logic into a number of different categories 
depending on the type of analysis they require. These MISCs determine the species, energy, and incidence direction of accepted particles, and they count the numbers of events that occur with various combinations of these parameters using selected accumulation times ranging from $1 \mathrm{~s}$ to 1 hour as appropriate for the expected count rates and the data storage allocated to EPI-Hi on the spacecraft recorder.

The flight software derives the atomic number of each detected ion by using the measured energy deposited in the detector in which the particle stopped $\left(E^{\prime}\right)$ and in the preceding detector $(\Delta E)$ together with the mean thickness penetrated in the $\Delta E$ detector, which depends on both the direction of incidence and the measured thickness characteristics of the detector. We determine the regions occupied by the tracks for each of the elements of interest, and also for electrons, based on the calculated instrument response validated using preflight accelerator calibrations and refined based on data collected in flight (see Sect. 4.10). We determine the incident energies of stopping particles by summing up the energies deposited in each detector and adding a small correction for the unmeasured energy loss in the telescope window. For ions we use the mass of the most abundant isotope having the derived atomic number in order to calculate the incident energy per nucleon, which is the quantity used for assigning the particle to a particular energy bin. For He nuclei having incidence directions for which the mass resolution is sufficient for resolving ${ }^{3} \mathrm{He}$ from ${ }^{4} \mathrm{He}$, we also calculate the mass of the detected isotope.

In addition to counts of different categories of events accumulated at various time cadences, we also accumulate the corresponding efficiency factors and live-times needed to correct the measured count rates. These rates and associated correction factors are the primary data returned by EPI-Hi.

We employ a prioritization scheme for selecting a sample of events to be returned with their complete set of measured pulse heights. We use these event data for validating the on-board analysis and for addressing specialized science topics. The number of events to be recorded is flexible and we adjust it depending on the volume of data that can be downlinked for a particular orbit.

We also measure and return a variety of housekeeping parameters including temperatures, voltages, and detector leakage currents. We have developed a second ASIC called the "HK Chip" for measuring these parameters. This chip also handles assorted other support functions and allows a significant reduction in the parts count on the telescope and DPU boards. For example, the HK Chip includes a high-precision DAC that provides the reference voltage required by the test pulsers in the PHASICs. As with the PHASIC, we have already fabricated and tested a non-rad-hard version of the HK Chip to validate the design that will be used for production of rad-hard flight parts by Aeroflex Inc.

\subsection{Gamma Rays and Neutrons}

The HET telescope can detect gamma rays and neutrons (see Sect. 1.6) since the central regions of $\mathrm{H} 3 \mathrm{~A}$ through $\mathrm{H} 3 \mathrm{~B}$ (see Fig. 39) provide a $1.2 \mathrm{~cm}^{3}$ sensitive volume of silicon almost completely surrounded by active material consisting of their guard regions together with $\mathrm{H} 2 \mathrm{~A}$ and $\mathrm{H} 2 \mathrm{~B}$. As a result, triggers of one or more central segments (with the surrounding detector elements in anti-coincidence) can be caused by $\sim 0.5-6 \mathrm{MeV}$ gamma-rays that Compton-scatter or pair-produce as well as by $\sim 2-20 \mathrm{MeV}$ neutrons that undergo nuclear reactions such as $\left.{ }^{28} \mathrm{Si}(n, p)\right)^{28} \mathrm{Al}$ or ${ }^{28} \mathrm{Si}(n, \alpha){ }^{25} \mathrm{Mg}$ (Mewaldt et al. 1977).

\subsection{Calibration Plan}

Onboard determinations of the composition and energy spectra of ions ranging from $\mathrm{H}$ to $\mathrm{Ni}$ and of electrons require precise calibrations of the response of the LET and HET detectors 
Fig. 39 Cross section of the HET silicon detector active areas illustrating how detectors are used for detection of gamma rays and neutrons. For these measurements, all of the orange-shaded detector segments are used in anticoincidence to reject incident charge particles. The green-shaded segments are used to record energy losses from charged particles produced when neutrals interact in the interior of the telescope. Lines indicate a neutral particle (upper dashed line) that scatters producing a charged particle (solid line) plus a second neutral (lower dashed line) that escapes from the telescope. Only the charged particle is detected
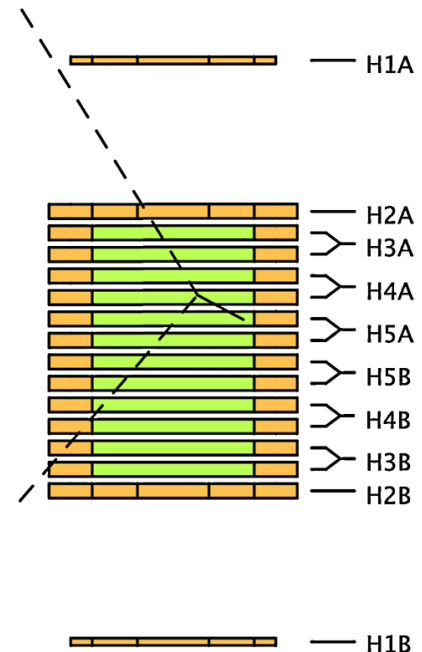
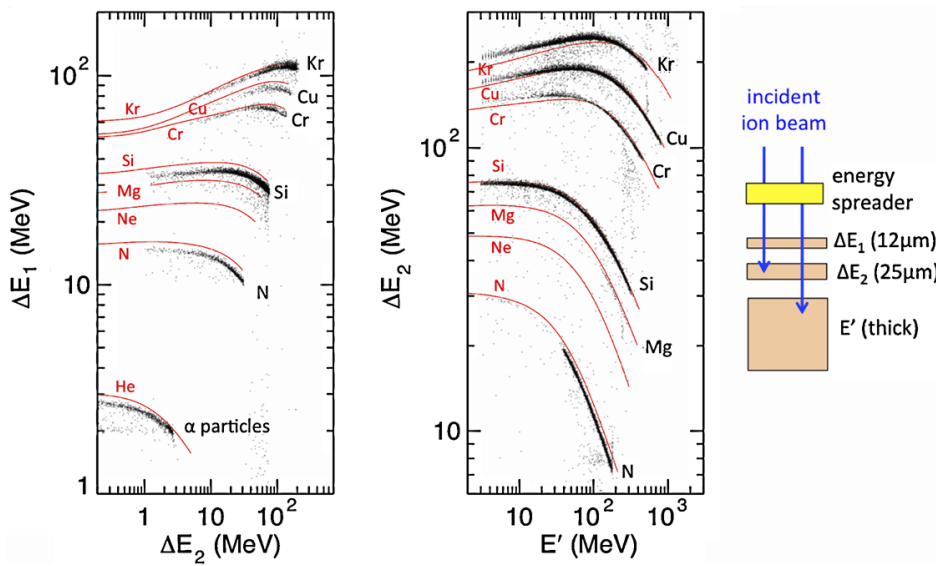

Fig. 40 Data from a heavy-ion test of a detector telescope consisting of one L0 detector and one L1 detector in front of a thick residual energy detector, as illustrated in the sketch at the right. A "cocktail beam" containing a variety of heavy ions was obtained from the 88-inch cyclotron at the Lawrence Berkeley National Laboratory. Red curves show the response calculated from a published range-energy relation. Accelerator data of the type shown can be used to better calibrate the locations of the response tracks

and electronics over a broad range of operating temperatures and incident particle energies, directions, and intensities. To carry out the particle identification procedure described in Sect. 4.8 (see Fig. 33) we must know precisely the mean thickness of all detector segments, the transfer function from pulse-height to energy in each of the PHASIC circuits, and rangeenergy relations for all of the ions of interest.

We performed an initial test of several LET L0 and L1 detectors in October 2013 at Berkeley Lab's 88-inch cyclotron. The measured narrowness of the tracks in Fig. 40 demonstrates that both L0 and L1 have the required energy resolution and thickness uniformity to identify elements up to at least $\operatorname{Kr}(Z=36)$. We also measure the thickness characteristics of the 12 and $25 \mu \mathrm{m}$ detectors in the laboratory with collimated alpha-particle sources. Our 
experience with SSDs on STEREO indicates that the 500 and $1000 \mu \mathrm{m}$ detectors for EPI-Hi will be sufficiently uniform to preclude the need to carry out thickness uniformity studies of these devices. From Fig. 40 we conclude that existing heavy-ion range-energy relations require only minor adjustments to calculate the locations of the element tracks.

The on-board particle identification system also requires accurate pre-launch and in-flight measurements of the gain and offset of each PHASIC's dual-gain PHA. Since EPI-Hi may experience temperature variations over the orbit, we will measure the temperature coefficients of each flight PHASIC. Fortunately, the PHASICs are very stable over temperature, with gain temperature coefficients of $<50 \mathrm{ppm} /{ }^{\circ} \mathrm{C}$, and offset temperature coefficients of $<0.1$ channel $/{ }^{\circ} \mathrm{C}$. In addition, we will perform periodic in-flight calibrations of each $\mathrm{ADC}$ using the test pulsers built into the PHASICs.

For the electron calibrations for LET and HET, we model the telescope responses using the Geant4 simulation package (http://geant4.web.cern.ch/geant4/) and spot-check the simulation results using laboratory beta-decay sources such as ${ }^{106} \mathrm{Ru}$, which has a maximum electron energy of $3.54 \mathrm{MeV}$.

We are planning a final end-to-end test of EPI-Hi at the Michigan State University National Superconducting Cyclotron Laboratory (NSCL), which can accelerate ${ }^{58} \mathrm{Ni}$ ions up to $160 \mathrm{MeV} /$ nuc. By fragmenting ${ }^{58} \mathrm{Ni}$ and a lighter beam like $\mathrm{Ne}$ in a thick target, we can measure the $\Delta E$ versus $E^{\prime}$ response tracks for elements from $\mathrm{H}$ to Ni (Mewaldt et al. 2008b). We can also test and calibrate the instrument's performance under high-rate conditions at NSCL.

Following launch, we will collect and telemeter energetic particle data to evaluate the performance of the LET and HET on-board particle identification and update the calibration parameters as necessary. Data collected in flight will also be used for intercalibrating EPI-Hi with EPI-Lo.

\subsection{Environmental Tests}

We have subjected prototypes of the thin silicon detectors, L0 and L1, to selected environmental tests since these detectors are not only a new technology development but will also experience some particularly harsh conditions. We constructed a mechanical model of the L0 detector having a thin silicon membrane of the same diameter but only $80 \%$ of the L0 thickness, and subjected it to a severe acoustic test that went up to an overall sound pressure level of $143 \mathrm{~dB}$. The model came through this test undamaged.

Although the radiation specification used for most components on SPP is relatively modest, $\sim 20$ to $40 \mathrm{krad}$ for total ionizing dose and $\sim 1 \times 10^{11}$ to $2 \times 10^{11}$ protons $/ \mathrm{cm}^{2}$ fluence for displacement damage, the thin silicon detectors are, of necessity, protected by much less shielding than a typical electronic subassembly. We tested prototype thin silicon detectors up to $10 \mathrm{Mrad}$ total dose using the ${ }^{60} \mathrm{Co}$ source in the Jet Propulsion Laboratory (JPL) highdose-rate test facility and up to a proton fluence of $10^{13} \mathrm{~cm}^{-2}$ at the University of California Davis cyclotron. The devices survived these extreme tests and showed negligible degradation of their response when tested with alpha particle sources. The increase in the detector leakage currents due to the displacement damage was significant, with the L0 current going from $\sim 5 \mathrm{nA}$ to $\sim 0.7 \mu \mathrm{A}$ after the proton exposure, but was in good agreement with the expected increase (Lutz 1999). This change is well within the design limits of the EPI-Hi bias system and front-end electronics.

The characteristics of the population of high-speed dust particles orbiting close to the Sun are poorly known, but these particles could present a significant risk of damaging sensitive exposed surfaces such as the thin windows and thin front detectors in LET. Because of this, 
we have already carried out initial tests of prototypes of the polyimide windows at dust accelerators in Heidelberg, Germany and at the University of Colorado. Dust particles with kinetic energies approximating those expected close to the Sun were able to penetrate the few- $\mu \mathrm{m}$ thicknesses of these windows, but did not cause any windows to rupture. We also performed a preliminary test of the effect of dust impacts on a thin silicon detector and determined that the detector was still operable after the test. More detailed dust testing is continuing.

We have also designed the coincidence logic for the LET telescopes to improve their robustness against damage due to dust impacts. As discussed above (Sect. 4.3), we can select events for analysis in LET on the basis of a coincidence between L1 and L2, without requiring a signal from $\mathrm{L} 0$. Thus if the $\mathrm{L} 0$ detector were to fail due either to a direct dust impact or to a large noise increase as might occur if the windows were to suffer enough damage to allow a significant flux of light to impinge on L0, operation could continue with a somewhat higher LET threshold energy.

\section{Science Operations, Data Processing, and Data Products}

\subsection{Software/Instrument Modes}

The SPP prime mission is composed of 24 highly elliptical, heliocentric orbits with decreasing orbital periods from 168 days for orbit 1 , settling into an $\sim 88$ day orbit period midway through the mission. Each orbit is broken into two distinct periods, the Solar Encounter period (inside 0.25 $\mathrm{AU}$ ) and the Cruise/Downlink period (outside 0.25 AU). For each orbit, the solar encounter duration is $\sim 10-11$ days. During the solar encounters all instruments across the SPP payload are powered on and continuously collecting science data (for more on instrument operations, see below). Although limited commanding is supported during the encounter period, spacecraft commanding is not nominally planned during this period. All normal spacecraft activities during the encounter period will execute via time-tagged or event-tagged onboard command sequences.

The ISIS instruments are planned to operate similarly in two operational modes:

- Normal Science Mode

- Spacecraft-Sun Distance: $R \leq 0.25 \mathrm{AU}$ ( $R$ is heliocentric radius)

- Full nominal power

- High data collection rate and burst data (EPI-Lo)

- Low-rate Science Mode

- Spacecraft-Sun Distance: $R>0.25$ AU

- Full power when not downlinking and whenever possible

- Reduced data collection rate (fits within ISIS telemetry allocation)

- Commanding window scheduled late in the series of telemetry passes, although it may not be used every orbit

The instruments are capable of switching between these routine modes autonomously, based on information received once-per-second from the spacecraft. EPI-Hi has no "Burst" mode. During "Burst" periods EPI-Lo sends telemetry to the spacecraft at higher rates.

At least three special operational modes are currently envisioned:

- Software upload mode

- Calibration Science mode

- Safe mode 
During Calibration Science mode, samples of PHA event data are accumulated and returned to validate onboard assignments of species, energy, and incidence angle, and for assessing instrument backgrounds. This mode is activated outside 0.25 AU by command several times early in the mission when sufficiently high intensities of solar energetic particles are present. Following ground analysis of data gathered in Calibration Science mode, onboard tables and parameters will be updated as needed. These tables and parameters are pre set, based on the pre-flight calibrations, but it is likely that some fine-tuning will be needed. We expect to upload new tables about 3 weeks into the statistics gathering period, and then use the remaining time to verify the new tables or make further updates as needed. In safe mode, the ISIS instruments return to a "quiet" state, awaiting commands from the ground. This mode is reserved for instrument I\&T, commissioning, and instances where the flight software encounters a fault or out-of-limit condition.

Particle events are sporadic so the goal is to approach $100 \%$ coverage. Whenever possible, ISIS instruments should be on and taking data.

The ISIS team streamlines instrument operation by minimizing commanding and mode changes. The spacecraft telemetry file system is used to prioritize data downlink:

- First priority: Housekeeping for health and safety

- Second priority: Snapshot data to identify important time periods (immediately relayed to other SPP instruments)

- Third priority: Full Science Data (rates at different cadences and event data)

Because solar encounters occur frequently, the team has a three-step hierarchy of planned commands for near-term and longer-term changes. Specifically, command loads will be planned for solar passes $N, N+1$, and $N+2$ where pass $N$ corresponds to the next solar pass. This pro-active planning process will allow for any needed adjustments based on recent data, while ensuring that plans are in place well in advance of imminent passes.

\subsection{ISIS Science Operations Center}

The ISIS SOC is housed at the University of New Hampshire (UNH) with heritage based on the Interstellar Boundary Explorer (IBEX) Mission. The primary functions of the ISIS SOC functions include planning, commanding, monitoring instrument health and safety, and producing and archiving data products. The key requirements of the ISIS SOC are:

- Provide public "quick look" data 60 days after downlink (a less stringent requirement of 6 months for quick look data applied for the first 3 orbits)

- Provide public science data 6 months after downlink

- Share science/engineering/ancillary data among the SPP team

- Archive all telemetry, data, software and documents for mission +1 year

- Place all software and data in a final, deep archive by 12 months after the end of the mission

- Implement SPP project approved security on SOC computers

- Receive "remote SOC notification" of instrument fault conditions as detected by the spacecraft

The ISIS SOC has a common framework that flows from Ground Support Equipment Operations Systems (GSEOS) through development of the engineering models, flight models, I\&T, and flight operations. The common framework allows the ISIS SOC to develop as the team builds and learns to operate the instruments. It is important to have cross-coordination and cross-calibration, to the greatest degree possible. The ISIS SOC participates throughout 


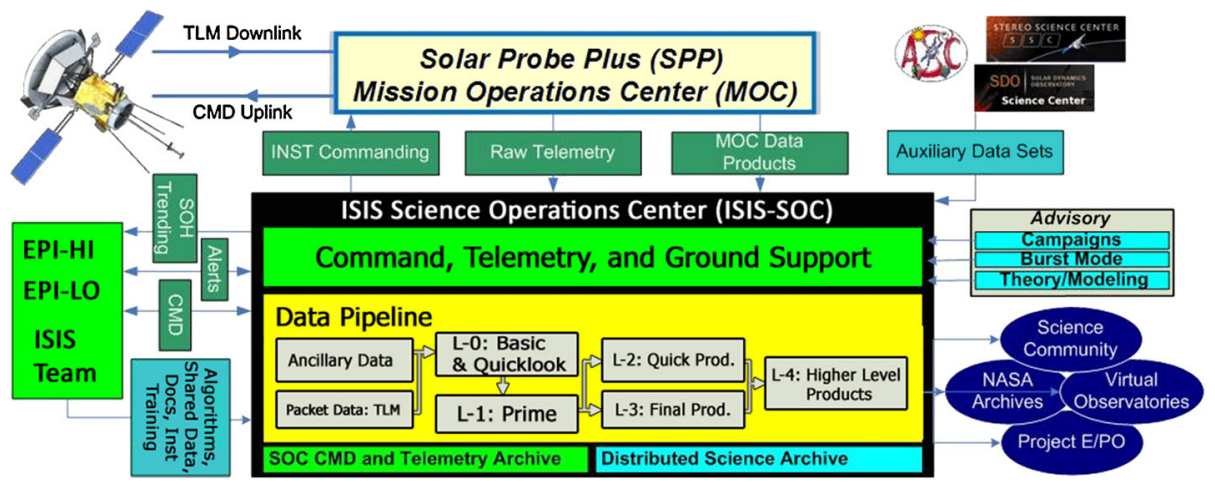

Fig. 41 Data flow through the ISIS-SOC housed at UNH. The ISIS SOC runs the data pipeline to produce data products (Levels 1-4) and houses the commanding center for ISIS. The ISIS and SPP teams aid the ISIS SOC for algorithm development, input concerning commanding, alert checking, and state-of-health trending. The data at the output of the ISIS SOC pipeline is delivered to the science community, NASA archives, virtual observatories and SPP project Education and Public Outreach centers

instrument characterization, calibration, I\&T, and flight. The ISIS SOC comprises a Command, Telemetry and Ground (CTG) group, a Science Data group, the pipeline and other analysis software, a Command and Telemetry Archive, and a Distributed Science Archive (Fig. 41). The connections between the SOC and MOC are illustrated in Fig. 42.

We apply the concept of "test as you fly, and fly as you test". The intention of "test as you fly" is to avoid improvisation during the critical flight phase. Operations plans, including instrument modes, will be modified based on experience from I\&T, commissioning, and early ops. However, we emphasize commonalities among all those phases rather than planning from the start to operate in very different modalities during testing and flight.

Instrument Ground Support Equipment (GSEs) fulfils two critical roles by providing a direct connection to instruments for ground testing and flight-like modes so that the spacecraft simulator can be used to test operations. The GSE flight-mode operation will be incorporated into the SOC GSEOS system to provide a nearly identical "look and feel" to the instrument/SOC teams during testing, I\&T, commissioning and normal flight operations.

The ISIS SOC CTG group is responsible for uploading instrument commands, validating command requests from the instrument teams, generating coordinated command sequences, receiving science and ancillary data from the MOC, monitoring instrument state-of-health, responding to alerts in coordination with the SPP instrument teams, processing raw science and ancillary data (including housekeeping), producing time-information for instrument data and housekeeping, and supporting ground testing and routine operations from pre-flight to post-flight. The ISIS SOC is staffed by a small team responsible for day-to-day operations, with operators brought on-board early in the project and operating the instruments during I\&T. The command and telemetry database developed during I\&T is used for flight operations.

The SOC CTG group checks instrument health and safety limits throughout testing, I\&T, commissioning, and flight. GSEOS is used at ISIS SOC for health and safety monitoring during contacts. The ISIS SOC also plans to develop specific ISIS software for health and safety checking, and SPP-accessible web pages with state-of-health checks and long-term trending. Housekeeping and status data are processed as soon as possible after receipt (within 3 days) and posted for viewing for the ISIS team through an ISIS SOC website. The house- 


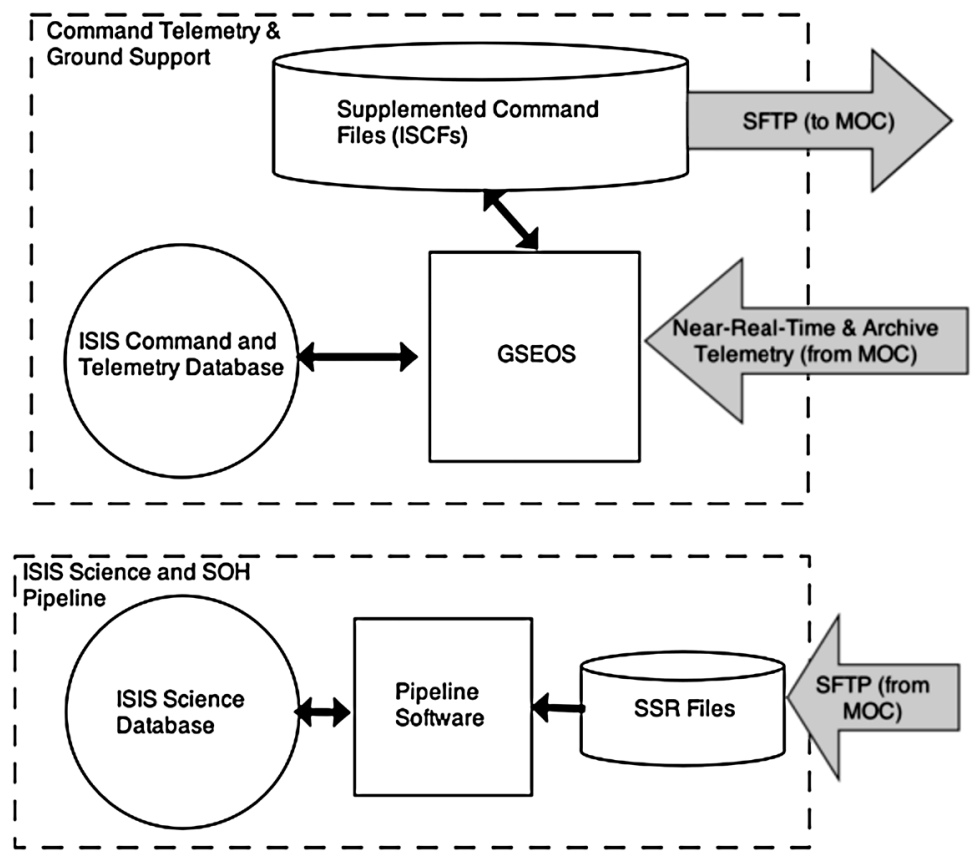

Fig. 42 Secure ftp (SFTP) connections to the SPP Mission Operations Center (MOC) at APL provides for transfer to the MOC of command files and transfer from the MOC of instrument telemetry. A near-real-time connection between the SOC and MOC to GSEOS provides for near-real-time instrument commanding and instrument monitoring

keeping and status data are downloaded first after passes, and provide critical information from which to build command loads in subsequent passes.

The ISIS SOC Science Data (SDG) group manages the repository for processing software and supports the SPP team throughout the project by processing Level 0, 1, 2, 3 and 4 (L-0 to L-4) data products; developing data analysis tools in coordination with the instrument teams and SPP communities; generating summary and quick-look plots; consolidating SPP data products with auxiliary data sets; serving data and analysis software to SPP communities; providing a center for scientific interchange meetings throughout the project; and developing a database of models and derived quantities.

The library of analysis software is maintained in a version control system downloadable to other sites maintained within the team. The analysis software is written in platformindependent languages such as C, IDL and Java for the pipeline software. Tools such as Mission Independent Data Layer (MIDL; http://sd-www.jhuapl.edu/MIDL/) and IDL will be available for visualization and analysis. Most processes within the ISIS SOC will be partially automated using Unix/Shell scripts that launch executables with clearly specified I/O.

Three types of software support the creation and analysis of science data for ISIS: software for integrating and testing of the instruments, ISIS-SOC components for processing telemetry into calibrated data products, and visualization tools for displaying and manipulating science data products. Data processing algorithms incorporate straightforward routines for decommutation, particle identification and application of calibration data. All formal pipeline processing is performed at the ISIS SOC. 
The SOC pipeline executes telemetry processing modules to be developed in coordination with the EPI-Lo and EPI-Hi teams. The processing modules convert telemetry into Level 1 data products, and the modules interact with the pipeline through a simple interface. UNH hosts the SOC web site, which serves calibrated data to science data centers, virtual observatories, and the public. The SOC will ensure that the team has rapid access to data.

The ISIS team is fully committed to an open and timely data release to maximize the usage and usability of the ISIS measurements from the SPP mission. The availability of all ISIS data will be fully compliant with the science data policy defined in the NASA Heliophysics Science Data Management Policy. After completion of in-orbit commissioning and cross-calibration, the SOC delivers data products. The ISIS team retains the data internally for only the time period necessary to ensure that the data are properly processed, calibrated, and verified. The ISIS Team and SOC strive to make this period as short as possible after receipt of the original science telemetry and auxiliary orbit, attitude and spacecraft status information.

The ISIS SOC also centrally creates metadata and handles data archiving deliveries. Crucial to making the archiving cost effective is ensuring that all ISIS data products are created archive-ready. This will involve some data design work by the teams early on to coordinate data formats, layouts, and metadata standards. This will enable the data to be released to the long-term archive with no changes. Solar Probe Plus falls under the purview of the Heliophysics Data Policy, which recommends the use of the CDF data format and the Space Physics Search Archive and Extract (SPASE) metadata standard. The public archive for the mission is the Space Physics Data Facility (SPDF) at the Goddard Space Flight Center.

\subsection{ISIS Data Products}

EPI-Hi and EPI-Lo data products are detailed in Table 7. Level 0 data products are essentially raw, whereas Level 1 data products are validated and released to the science community after the validation process is complete. Level 2 data products are being defined.

The ISIS SOC also provides to the public a quick-look processed version of science data within 6 months of downlink for the first three orbits after launch and within 60 days of downlink thereafter, including:

- EPI-Lo spectrograms of $<1 \mathrm{MeV}$ electron counts/data-interval as a function of time, at less than 1 hour cadence, with 4 or more energy bins.

- EPI-Lo spectrograms of $<1 \mathrm{MeV} /$ nuc total ion or single species counts/data-interval as a function of time, at a $<1$ hour cadence, with 4 or more energy bins.

- EPI-Hi plots of 1-hour averages of four rates: 1-5 MeV electrons; 2-10 MeV protons; 10-50 MeV protons; and 4-40 MeV/nuc HiZ (6 $\leq Z \leq 28)$.

Data verification and validation are partly automated, but also require evaluation by ISIS scientists. Verification and validation processes are coordinated by the ISIS Science Data group. After the 3-orbit period, all ISIS data products will be made available to the public no later than 6 months after downlink. The ISIS team also defines and produces additional high-level products as needed. For example, Solar Proton Event lists and Co-rotating Interaction Region (CIR) event lists will likely be produced. The list of such products will evolve throughout the mission as new regimes are discovered. The ISIS team will fully engage with the SPP team as a whole to define and provide new high-level products throughout the mission. These products along with the most accurate data and a complete set of ISIS data products will be regularly released to the SPDF.

The coordination of data with other SPP teams and with information from outside missions and ground-based data will be carried out through the SOC. A series of Level 2 data 
Table 7 Data products level, description, latency and any dependencies

\begin{tabular}{|c|c|c|c|}
\hline Data level & Description & Latency & Users \\
\hline L0 status data & $\begin{array}{l}\text { Snapshots of EPI-Lo and EPI-Hi data } \\
\text { through the orbit }\end{array}$ & $\begin{array}{l}\text { Downloaded first } \\
\text { following each orbit }\end{array}$ & $\begin{array}{l}\text { ISIS instrument } \\
\text { teams }\end{array}$ \\
\hline L0 housekeeping & $\begin{array}{l}\text { EPI-Hi and EPI-Lo voltages, currents, } \\
\text { temperatures, rate monitors }\end{array}$ & $\begin{array}{l}\text { Downloaded first } \\
\text { following each orbit }\end{array}$ & $\begin{array}{l}\text { ISIS instrument } \\
\text { teams }\end{array}$ \\
\hline $\begin{array}{l}\text { L0 command } \\
\text { response data }\end{array}$ & $\begin{array}{l}\text { Data products that detail results of specific } \\
\text { commands }\end{array}$ & $\begin{array}{l}\text { Data delivered asap } \\
\text { after tests }\end{array}$ & $\begin{array}{l}\text { ISOC and ISIS } \\
\text { instrument } \\
\text { teams }\end{array}$ \\
\hline $\begin{array}{l}\text { L0 memory } \\
\text { dumps }\end{array}$ & $\begin{array}{l}\text { EPI-Hi and EPI-Lo will perform slow (bit/s) } \\
\text { memory dumps periodically }\end{array}$ & $\begin{array}{l}\text { Data delivered after } \\
\text { each pass }\end{array}$ & $\begin{array}{l}\text { ISOC and ISIS } \\
\text { instrument } \\
\text { teams }\end{array}$ \\
\hline $\begin{array}{l}\text { L0 EPI-Hi events } \\
(Z \& E)\end{array}$ & $\begin{array}{l}\text { Sample of nuclear charge }(Z) \text { and kinetic } \\
\text { energy } E \text { of individual particles at } \\
\text { processing rates of }>1000 \text { particles/sec }\end{array}$ & $\begin{array}{l}\text { Data delivered after } \\
\text { each pass }\end{array}$ & $\begin{array}{l}\text { ISOC and ISIS } \\
\text { instrument } \\
\text { teams }\end{array}$ \\
\hline $\begin{array}{l}\text { L0 EPI-Hi } Z \text { vs. } \\
E \text { matrices }\end{array}$ & $\begin{array}{l}\text { Each of the processed "events" is sorted into } \\
\text { several } Z \text { vs. } E \text { matrices }\end{array}$ & $\begin{array}{l}\text { Data delivered after } \\
\text { each pass }\end{array}$ & $\begin{array}{l}\text { ISOC and ISIS } \\
\text { instrument } \\
\text { teams }\end{array}$ \\
\hline $\begin{array}{l}\text { L0 EPI-Hi } Z \text { vs. } \\
E \text { vs. direction } \\
\text { matrices }\end{array}$ & $\begin{array}{l}Z \text { vs. } E \text { matrices accumulated over multiple } \\
\text { look directions }\end{array}$ & $\begin{array}{l}\text { Data delivered after } \\
\text { each pass }\end{array}$ & $\begin{array}{l}\text { ISOC and ISIS } \\
\text { instrument } \\
\text { teams }\end{array}$ \\
\hline $\begin{array}{l}\text { L0 EPI-Lo } \\
\text { electron rates }\end{array}$ & Electron rates in angle and energy bins & $\begin{array}{l}\text { Data delivered after } \\
\text { each pass }\end{array}$ & $\begin{array}{l}\text { ISOC and ISIS } \\
\text { instrument } \\
\text { teams }\end{array}$ \\
\hline $\begin{array}{l}\text { L0 EPI-Lo ion } \\
\text { rates }\end{array}$ & $\begin{array}{l}\text { Proton, He and heavy ion rates in angle and } \\
\text { energy bins }\end{array}$ & $\begin{array}{l}\text { Data delivered after } \\
\text { each pass }\end{array}$ & $\begin{array}{l}\text { ISOC and ISIS } \\
\text { instrument } \\
\text { teams }\end{array}$ \\
\hline $\begin{array}{l}\text { L0 EPI-Lo PHA } \\
\text { events }\end{array}$ & $\begin{array}{l}\text { Complete information on select events. } \\
\text { Cadence of } 0.1 \mathrm{~s}<0.25 \mathrm{AU} \text { and } 5 \mathrm{~s}> \\
0.25 \mathrm{AU}\end{array}$ & $\begin{array}{l}\text { Data delivered after } \\
\text { each pass }\end{array}$ & $\begin{array}{l}\text { ISOC and ISIS } \\
\text { instrument } \\
\text { teams }\end{array}$ \\
\hline $\begin{array}{l}\text { L0 EPI-Lo } \\
\text { TOF-only events }\end{array}$ & $\begin{array}{l}\text { TOF information allowing detailed species } \\
\text { separation }\end{array}$ & $\begin{array}{l}\text { Data delivered after } \\
\text { each pass }\end{array}$ & $\begin{array}{l}\text { ISOC and ISIS } \\
\text { instrument } \\
\text { teams }\end{array}$ \\
\hline $\begin{array}{l}\text { L1 EPI-Lo } \\
\text { events, rates }\end{array}$ & $\begin{array}{l}\text { Products similar to EPI-Lo L0 data } \\
\text { products, however validated and time-sorted } \\
\text { with redundancies removed }\end{array}$ & $\begin{array}{l}\text { Data accumulated } \\
\text { after each pass }(N) \\
\text { and made available to } \\
\text { public in pass }(N+1)\end{array}$ & $\begin{array}{l}\text { SPP science } \\
\text { community }\end{array}$ \\
\hline $\begin{array}{l}\text { L1 EPI-Lo } \\
\text { particle } \\
\text { intensities }\end{array}$ & $\begin{array}{l}\text { Absolute intensities (in units of particles } \\
\left(\mathrm{cm}^{-2} \mathrm{sr}^{-1} \mathrm{~s}^{-1}[\mathrm{MeV} / \mathrm{nuc}]^{-1}\right) \text { for ion } \\
\text { species and in electrons } \\
\left(\mathrm{cm}^{-2} \mathrm{sr}^{-1} \mathrm{~s}^{-1} \mathrm{MeV}^{-1}\right) \text { for electrons). } \\
\text { These are produced from the L0 data }\end{array}$ & $\begin{array}{l}\text { Data accumulated } \\
\text { after each pass }(N) \\
\text { and made available to } \\
\text { public in pass }(N+1)\end{array}$ & $\begin{array}{l}\text { SPP science } \\
\text { community }\end{array}$ \\
\hline $\begin{array}{l}\text { L1 EPI-Hi } \\
\text { events, rates, } \\
\text { matrices }\end{array}$ & $\begin{array}{l}\text { Products similar to EPI-Hi L0 data } \\
\text { products, however validated and time-sorted } \\
\text { with redundancies removed }\end{array}$ & $\begin{array}{l}\text { Data accumulated } \\
\text { after each pass }(N) \\
\text { and made available to } \\
\text { public in pass }(N+1)\end{array}$ & $\begin{array}{l}\text { SPP science } \\
\text { community }\end{array}$ \\
\hline $\begin{array}{l}\text { L1 EPI-Hi LET } \\
\text { and HET particle } \\
\text { intensities }\end{array}$ & $\begin{array}{l}\text { Absolute intensities (in units of particles } \\
\left(\mathrm{cm}^{-2} \mathrm{sr}^{-1} \mathrm{~s}^{-1}[\mathrm{MeV} / \mathrm{nuc}]^{-1}\right) \text { for ion } \\
\text { species and in electrons } \\
\left(\mathrm{cm}^{-2} \mathrm{sr}^{-1} \mathrm{~s}^{-1} \mathrm{MeV}^{-1}\right) \text { for electrons) }\end{array}$ & $\begin{array}{l}\text { Data accumulated } \\
\text { after each pass }(N) \\
\text { and made available to } \\
\text { public in pass }(N+1)\end{array}$ & $\begin{array}{l}\text { SPP science } \\
\text { community }\end{array}$ \\
\hline
\end{tabular}


Table 7 (Continued)

\begin{tabular}{|c|c|c|c|}
\hline Data level & Description & Latency & Users \\
\hline $\begin{array}{l}\text { L1 EPI-Hi } \\
\text { expanded event } \\
\text { data }\end{array}$ & $\begin{array}{l}\text { These data are produced for a sample of the } \\
\text { individual ions/electrons that trigger the } \\
\text { instrument. A priority system will ensure } \\
\text { that all species, energies, and directions are } \\
\text { sampled }\end{array}$ & $\begin{array}{l}\text { Data accumulated } \\
\text { after each pass }(N) \\
\text { and made available to } \\
\text { public in pass }(N+1)\end{array}$ & $\begin{array}{l}\text { SPP science } \\
\text { community }\end{array}$ \\
\hline L2 data sets & $\begin{array}{l}\text { Higher level products that combine various } \\
\text { data products. Examples include data } \\
\text { products time-ordered with solar wind and } \\
\text { magnetic field data, element abundance } \\
\text { ratios, fits to particle anisotropy data, and } \\
\text { particle intensities associated with particular } \\
\text { solar events }\end{array}$ & $\begin{array}{l}\text { Data accumulated } \\
\text { after each pass }(N) \\
\text { and made available to } \\
\text { public in pass }(N+1)\end{array}$ & $\begin{array}{l}\text { SPP science } \\
\text { community }\end{array}$ \\
\hline
\end{tabular}

products will be generated including coordinated SPP products (solar wind, magnetic field, energetic particles), SPP products coordinated with 1 AU data sets (e.g., STEREO, ACE, L1 observers, GOES), data sets from spacecraft beyond $1 \mathrm{AU}$, and data sets from other missions inside $1 \mathrm{AU}$ such as Solar Orbiter. Also critical to understanding the coronal environment are data sets from remote and ground based observations. Potential field models, for example, provide important information about the connection between features observed by SPP and possible source regions on the Sun. ISIS Level 2 data sets therefore incorporate not only data but also results of data-based models such as potential field models. Level 3 and higher level data products are developed based on scientific needs, incorporation of models, and physical context of the environment.

\section{Summary and Conclusions}

The ISIS investigation on the Solar Probe Plus mission has been optimized to explore the sources of energetic particles near the Sun and mechanisms that accelerate and transport them as their distributions evolve outward into interplanetary space. ISIS will finally answer the fundamental questions of the origins, acceleration, and transport of solar energetic particles and provide new discovery science.

ISIS accomplishes this with its two electrically independent instruments that together measure energetic particles over the broad energy range from $\sim 0.02$ to $\sim 200 \mathrm{MeV} /$ nuc for ions and $\sim 0.025-6 \mathrm{MeV}$ for electrons. The instruments are mounted together on a common ISIS bracket that allows viewing as close to the spacecraft heat shield umbra as possible, which ensures broad pitch angle observations across these energy ranges. Science planning and operations are carried out through the ISIS Science Operation Center, which also produces the combined ISIS science products and coordinates data with the other SPP investigation science centers, including science teams from many other space missions, such as Solar Orbiter, ground-based observers, and others. The data products will optimize the scientific productivity and output not just from the SPP investigation teams, but from the entire Heliophysics community.

EPI-Lo is a high-heritage, TOF-based mass spectrometer that measures energetic electron $(25-1000 \mathrm{keV})$ and ion spectra $(\sim 0.02 \mathrm{MeV} /$ nuc to a maximum energy of $15 \mathrm{MeV}$ for most elements). By resolving all major heavy ion species and ${ }^{3} \mathrm{He}$ and ${ }^{4} \mathrm{He}$ over much of this energy range in multiple directions, EPI-Lo provides the required data across the critical 
energy range from suprathermal energies $(\sim 20 \mathrm{keV} / \mathrm{nuc})$ up to the lower portion of EPI-Hi energy range with a single instrument.

EPI-Hi measures energetic particle spectra, composition, and angular distributions using the $d E / d x$ vs. $E$ technique in a sensor system consisting of a double-ended High Energy Telescope (HET), and two Low Energy Telescopes (LETs), one double-ended (LET1) and one single-ended (LET2). Together these EPI-Hi telescopes cover $\sim 1$ to at least $100 \mathrm{MeV}$ for protons, and higher for heavy elements, and $\sim 0.5$ to $6 \mathrm{MeV}$ for electrons.

Together, coordinated observations from EPI-Hi and EPI-Lo, along with the centralized ISIS SOC and broader ISIS science team, are configured to revolutionize our understanding of the critical energetic particle environment that SPP will encounter as it explores the inner heliosphere progressively closer to the Sun.

In conclusion, Solar Probe Plus is the key Heliophysics mission for understanding the solar corona and inner heliosphere. Only by repeatedly traveling very close to the Sun is it possible to observe the regions where solar energetic particles are born, energized, and injected into the interplanetary environment. Only by making these critical observations is it possible to understand the origins, energization and transport of solar energetic particles, which drive key aspects of space weather and pose a significant risk for human exploration of space and for our increasingly technological space-based infrastructure. The Integrated Science Investigation of the Sun will make these unique observations and develop the critical scientific advances and knowledge needed to understand them.

\section{Acronym List}

\begin{tabular}{|c|c|}
\hline$\AA$ & Angstrom \\
\hline ACR & Anomalous Cosmic Ray \\
\hline $\mathrm{ADC}$ & Analog-to-Digital Converter \\
\hline AMU & Atomic Mass Unit \\
\hline APD & Analog Peak Detect \\
\hline APL & John Hopkins University Applied Physics Laboratory \\
\hline ASIC & Application-Specific Integrated Circuit \\
\hline ASSY & Assembly \\
\hline AU & Astronomical Unit \\
\hline B & Local Magnetic Field \\
\hline Berkeley Lab & Lawrence Berkeley National Laboratory \\
\hline $\mathrm{C} \& \mathrm{DH}$ & Command and Data Handling \\
\hline Caltech & California Institute of Technology \\
\hline $\mathrm{CBE}$ & Current Best Estimate \\
\hline CCSDS & Consultative Committee for Space Data Systems \\
\hline CFD & Constant-Fraction Discriminator \\
\hline CFDP & CCSDS file Delivery Protocol \\
\hline CIR & Corotating Interaction Region \\
\hline CMD & Command \\
\hline CME & Coronal Mass Ejection \\
\hline CMOS & Complementary metal-oxide-semiconductor \\
\hline $\mathrm{CNO}$ & Carbon, Nitrogen, and Oxygen \\
\hline Co-I & Co-Investigator \\
\hline
\end{tabular}




\begin{tabular}{|c|c|}
\hline CTG & Command, Telemetry and Ground \\
\hline DPU & Data Processing Unit \\
\hline ECC & Error Correction Code \\
\hline EEE & Electrical, Electronic, and Electromechanical \\
\hline EIS & Energetic Ion Spectrometer \\
\hline EM & Engineering Model \\
\hline EMC & Electromagnetic Compatibility \\
\hline EMI & Electromagnetic Interference \\
\hline ENA & Energetic Neutral Atom \\
\hline $\mathrm{EP}$ & Energetic Particle \\
\hline EPD & Energetic Particle Detector \\
\hline EPI-Hi & Energetic Particle Instrument for High Energies \\
\hline EPI-Lo & Energetic Particle Instrument for Low Energies \\
\hline EPS & Energetic Particle Spectrometer \\
\hline EUV & Extreme UltraViolet \\
\hline FAST & Fast Auroral Snapshot Explorer \\
\hline FEM & Finite Element Model \\
\hline FIELDS & Magnetic Field Suite \\
\hline FM & Flight Model \\
\hline FOV & Field of View \\
\hline FPGA & Field-Programmable Gate Array \\
\hline FSW & Fast Solar Wind \\
\hline FUV & Far Ultraviolet \\
\hline FWHM & Full Width Half Maximum \\
\hline GCR & Galactic Cosmic Ray \\
\hline GLE & Ground Level Event \\
\hline GN2 & High Purity Gaseous Nitrogen \\
\hline GSE & Ground Support Equipment \\
\hline GSEOS & Ground Support Equipment Operations Systems \\
\hline GSEP & Gradual Solar Energetic Particle \\
\hline GSFC & Goddard Space Flight Center \\
\hline $\mathrm{H} 1$ & Front detectors \\
\hline $\mathrm{H} 2$ & Outermost detectors \\
\hline $\mathrm{H} \#$ & SSDs in the HET, numbered from the entrance detector inward \\
\hline HENA & High Energy Neutral Atom \\
\hline HET & High Energy Telescope \\
\hline HK & Housekeeping \\
\hline HVPS & High Voltage Power Supply \\
\hline $\mathrm{I} / \mathrm{F}$ & Interface \\
\hline $\mathrm{I} / \mathrm{O}$ & Input/Output \\
\hline I\&T & Integration and Testing \\
\hline IBEX & Interstellar Boundary Explorer \\
\hline IBL & Idaho National Laboratory \\
\hline IDL & Interactive Data Language \\
\hline IMAGE & Imager for Magnetopause-to-Aurora Global Exploration \\
\hline IMF & Interplanetary Magnetic Field \\
\hline
\end{tabular}




\begin{tabular}{|c|c|}
\hline IMP-8 & Interplanetary Monitoring Platform- 8 \\
\hline IMPACT & In-situ Measurements of Particles and CME Transients \\
\hline INCA & Ion and Neutral Camera \\
\hline INST & Instrument \\
\hline IP & Interplanetary \\
\hline ISCFS & Instrument Supplemented Command Files \\
\hline ISEE-1 & International Sun Earth Explorer-1 \\
\hline ISEP & Impulsive Solar Energetic Particle \\
\hline ISIS & Integrated Science Investigation of the Sun \\
\hline ITF & Interface Transfer Frame \\
\hline JEDI & Jovian Energetic Particle Detector Instrument \\
\hline JPL & Jet Propulsion Laboratory \\
\hline L-\# & Level of data processing, L-1 is the least processed \\
\hline L\# & SSDs in the LET, numbered from the entrance detector inward \\
\hline LET & Low Energy Telescope \\
\hline LET1 & Double-ended Low Energy Telescope \\
\hline LET2 & Single-ended Low Energy Telescope \\
\hline LVDS & Low Voltage Differential Signaling \\
\hline LVPS & Low Voltage Power Supply \\
\hline $\mathrm{MCP}$ & Microchannel Plate \\
\hline MESSENGER & MErcury Surface, Space ENvironment, GEochemistry, and Ranging \\
\hline MHD & Magnetohydrodynamics \\
\hline MIDL & Mission Independent Data Layer \\
\hline MIMI & Magnetospheric Imaging Instrument \\
\hline MISC & Minimal Instruction Set Computer \\
\hline MLI & Multi-layer insulation \\
\hline MMS & Magnetospheric Multiscale \\
\hline MOC & Mission Operations Center \\
\hline MRAM & Magnetoresistive Random-Access Memory \\
\hline MSC & MacNeal-Schwendler Corporation \\
\hline MUX & Multiplexer \\
\hline NASA & National Aeronautics and Space Administration \\
\hline NRC & National Research Council \\
\hline NSCL & National Superconducting Cyclotron Laboratory \\
\hline nuc & Nucleon \\
\hline NuSTAR & Nuclear Spectroscopic Telescope Array \\
\hline PDR & Preliminary Design Review \\
\hline PDU & Power Distribution Unit \\
\hline PEPSSI & Pluto Energetic Particle Spectrometer Science Investigation \\
\hline $\mathrm{PH}$ & Pulse Height \\
\hline PHA & Pulse Height Analysis or Pulse Height Analyzer \\
\hline PHASIC & Pulse Height Analysis System Integrated Circuit \\
\hline PI & Principal Investigator \\
\hline PPS & Pulse-Per-Second \\
\hline PROM & Programmable Read-Only Memory \\
\hline PWA & Printed Wiring Assemblies \\
\hline
\end{tabular}




\begin{tabular}{|c|c|}
\hline PWB & Printed Wiring Board \\
\hline $\mathrm{Q} / \mathrm{M}$ & Charge-to-Mass ratio \\
\hline QDAC & Quad Digital-to-Analog Converter \\
\hline RBSPICE & Radiation Belt Storm Probes Ion Composition Experiment \\
\hline ROM & Rough Order-of-Magnitude \\
\hline Rs & Solar Radii \\
\hline RTD & Resistance Temperature Detector \\
\hline $\mathrm{S} / \mathrm{C}$ & spacecraft \\
\hline SATK & Structural Analysis Toolkit \\
\hline SDG & Science Data Group \\
\hline SEP & Solar Energetic Particle \\
\hline SEU & Single-Event Upset \\
\hline SFTP & Secure File Transfer Protocol \\
\hline SOC & Science Operations Center \\
\hline $\mathrm{SOH}$ & State-of-Health \\
\hline SPASE & Space Physics Search Archive and Extract \\
\hline SPDF & Space Physics Data Facility \\
\hline SPP & Solar Probe Plus \\
\hline SRAM & Static Random-Access Memory \\
\hline SSD & Solid-state Detector \\
\hline SSR & Solid-State Recorder \\
\hline SSW & Slow Solar Wind \\
\hline ST & Suprathermal \\
\hline STDT & Science and Technology Definition Team \\
\hline STEP & Suprathermal Energetic Particle telescope on the Wind spacecraft \\
\hline STEREO & Solar TErrestrial RElations Observatory \\
\hline SWEAP & Solar Wind Electrons Alphas and Protons \\
\hline SWE & Solar Wind Experiment on the Wind spacecraft \\
\hline SwRI & Southwest Research Institute \\
\hline TDC & Time-to-digital Conversion \\
\hline TID & Total Ionizing Dose \\
\hline TLM & Telemetry \\
\hline TMR & Triple Module Redundancy \\
\hline TOF & Time-of-flight \\
\hline TPS & Thermal Protection System \\
\hline $\mathrm{U}-\mathrm{Az}$ & University of Arizona \\
\hline U-Del & University of Delaware \\
\hline ULEIS & Ultra Low Energy Isotope Spectrometer \\
\hline $\mathrm{UNH}$ & University of New Hampshire \\
\hline UV & Ultraviolet \\
\hline WISPR & Wide Field Imager \\
\hline$Z$ & Nuclear Charge \\
\hline
\end{tabular}

Acknowledgements We are deeply indebted to all of the outstanding men and women who have made the Solar Probe Plus Mission and ISIS investigation a reality. These include members of the Solar Probe/Solar Probe Plus Science and Technology Definition Team (STDT), NASA headquarters personnel who have sup- 
ported and continue to support the mission, project team members at APL and GSFC, instrument team members at a variety of universities and other institutions across the country and around the world, and other members of the community who support this critical mission through their advisory work for the NRC and informally through many other means. We also thank Wendy Mills for the great job she did in assembling and editing this paper for the ISIS Team.

Open Access This article is distributed under the terms of the Creative Commons Attribution License which permits any use, distribution, and reproduction in any medium, provided the original author(s) and the source are credited.

\section{References}

S.P. Ahlen, Theoretical and experimental aspects of the energy loss of relativistic heavily ionizing particles. Rev. Mod. Phys. 52(1), 121 (1980)

J. Ambrosiano, W.H. Matthaeus, M.L. Goldstein, D. Plante, Test particle acceleration in turbulent reconnecting magnetic fields. J. Geophys. Res. 93, 14383-14400 (1988). doi:10.1029/JA093iA12p14383

G.B. Andrews, T.H. Zurbuchen, B.H. Mauk, H. Malcom, L.A. Fisk, G. Gloeckler, G.C. Ho, J.S. Kelley, P.L. Koehn, T.W. Lefevere, S.S. Livi, R.A. Lundgren, J.M. Raines, The energetic particle and plasma spectrometer instrument on the MESSENGER spacecraft. Space Sci. Rev. 131(1-4), 523-556 (2007). doi:10.1007/s11214-007-9272-5

M.J. Aschwanden, Particle acceleration and kinematics in solar flares-a synthesis of recent observations and theoretical concepts (Invited review). Space Sci. Rev. 101(1), 1-227 (2002). doi:10.1023/ A: 1019712124366

Bale et al., Space Sci. Rev. (2014, this issue)

H.V. Cane, R.A. Mewaldt, C.M.S. Cohen, T.T. von Rosenvinge, Role of flares and shocks in determining solar energetic particle abundances. J. Geophys. Res. 111(A6), A06S90 (2006). doi:10.1029/2005JA011071

B.D.G. Chandran, B. Li, B.N. Rogers, E. Quataert, K. Germaschewski, Perpendicular ion heating by lowfrequency Alfvén-wave turbulence in the solar wind. Astrophys. J. 720(1), 548-554 (2010). doi:10.1088/ 0004-637X/720/1/548

B.D.G. Chandran, D. Verscharen, E. Quataert, J.C. Kasper, P.A. Isenberg, S. Bourouaine, Stochastic heating, differential flow, and the alpha-to-proton temperature ratio in the solar wind. Astrophys. J. 776(1), 45 (2013). doi:10.1088/0004-637X/776/1/45, 8 pp.

S.R. Cranmer, A.A. van Ballegooijen, Can the solar wind be driven by magnetic reconnection in the Sun's magnetic carpet? Astrophys. J. 720(1), 824-847 (2010). doi:10.1088/0004-637X/720/1/824

S.R. Cranmer, A.A. van Ballegooijen, R.J. Edgar, Self-consistent coronal heating and solar wind acceleration from anisotropic magnetohydrodynamic turbulence. Astrophys. J. Suppl. 171(2), 520-551 (2007). doi:10.1086/518001

S. Dalena, A.F. Rappazzo, P. Dmitruk, A. Greco, W.H. Matthaeus, Test-particle acceleration in a hierarchical three-dimensional turbulence model. Astrophys. J. 783, 143 (2014). doi:10.1088/0004-637X/783/2/143

M.A. Dayeh, M.I. Desai, J.R. Dwyer, H.K. Rassoul, G.M. Mason, J.E. Mazur, Composition and spectral properties of the $1 \mathrm{AU}$ quiet-time suprathermal ion population during solar cycle 23. Astrophys. J. 693(2), 1588-1600 (2009). doi:10.1088/0004-637X/693/2/1588

M.I. Desai, G.M. Mason, J.R. Dwyer, J.E. Mazur, C.W. Smith, R.M. Skoug, Acceleration of 3He nuclei at interplanetary shocks. Astrophys. J. 553(1), L89-L92 (2001). doi:10.1086/320503

M.I. Desai, G.M. Mason, M.E. Wiedenbeck, C.M.S. Cohen, J.E. Mazur, J.R. Dwyer, R.E. Gold, S.M. Krimigis, Q. Hu, C.W. Smith, R.M. Skoug, Spectral properties of heavy ions associated with the passage of interplanetary shocks at 1 AU. Astrophys. J. 611(2), 1156-1174 (2004). doi:10.1086/422211

M.I. Desai, G.M. Mason, R.E. Gold, S.M. Krimigis, C.M.S. Cohen, R.A. Mewaldt, J.E. Mazur, J.R. Dwyer, Heavy-ion elemental abundances in large solar energetic particle events and their implications for the seed population. Astrophys. J. 649(1), 470-489 (2006). doi:10.1086/505649

P. Dmitruk, W.H. Matthaeus, N. Seenu, Test particle energization by current sheets and nonuniform fields in magnetohydrodynamic turbulence. Astrophys. J. 617(1), 667-679 (2004). doi:10.1086/425301

J.F. Drake, M. Swisdak, H. Che, M.S.A. Shay, Electron acceleration from contracting magnetic islands during reconnection. Nature 443(7111), 553-556 (2006). doi:10.1038/nature05116

D.C. Ellison, R. Ramaty, Shock acceleration of electrons and ions in solar flares. Astrophys. J. 298, 400-408 (1985). doi:10.1086/163623

L.A. Fisk, Motion of the footpoints of heliospheric magnetic field lines at the Sun: implications for recurrent energetic particle events at high heliographic latitudes. J. Geophys. Res. 101(A7), 15547-15554 (1996). doi:10.1029/96JA01005 
L.A. Fisk, G. Gloeckler, Particle acceleration in the heliosphere: implications for astrophysics. Space Sci. Rev. 173(1-4), 433-458 (2012). doi:10.1007/s11214-012-9899-8

Fox et al., Space Sci. Rev. (2014, this issue)

J. Giacalone, Particle acceleration at shocks moving through an irregular magnetic field. Astrophys. J. 624(2), 765-772 (2005). doi:10.1086/429265

J. Giacalone, J.R. Jokipii, J.E. Mazur, Small-scale gradients and large-scale diffusion of charged particles in the heliospheric magnetic field. Astrophys. J. 532(1), L75-L78 (2000). doi:10.1086/312564

J. Giacalone, Energetic charged particles associated with strong interplanetary shocks. Astrophys. J. 761(1), 28 (2012). doi:10.1088/0004-637X/761/1/28

N. Gopalswamy, H. Xie, S. Yashiro, S. Akiyama, P. Mäkelä, I.G. Usoskin, Properties of ground level enhancement events and the associated solar eruptions during solar cycle 23. Space Sci. Rev. 171(1-4), 23-60 (2012). doi:10.1007/s11214-012-9890-4

N. Gopalswamy, S. Yashiro, S. Krucker, G. Stenborg, R.A. Howard, Intensity variation of large solar energetic particle events associated with coronal mass ejections. J. Geophys. Res. Space Phys. 109(A12), A12105 (2004). doi:10.1029/2004JA010602

J.T. Gosling, The solar flare myth. J. Geophys. Res. 98(A11), 18,937-18,949 (1993). doi:10.1029/93JA01896

D.K. Haggerty, E.C. Roelof, Impulsive near-relativistic solar electron events: delayed injection with respect to solar electromagnetic emission. Astrophys. J. 579(2), 841-853 (2002). doi:10.1086/342870

Howard et al., Space Sci. Rev. (2014, this issue)

P.A. Isenberg, B.J. Vasquez, Kinetic models of fast solar wind driven by imbalanced ion cyclotron dissipation, in SOLAR WIND 13: Proceedings of the Thirteenth International Solar Wind Conference. AIP Conference Proceedings, vol. 1539 (2013), pp. 22-25. doi:10.1063/1.4810980

J.R. Jokipii, M.A. Lee, Compression acceleration in astrophysical plasmas and the production of $f(v) \propto v^{-5}$ spectra in the heliosphere. Astrophys. J. 713(1), 475-483 (2010). doi:10.1088/0004-637X/713/1/475

S.W. Kahler, A.H. McAllister, H.V. Cane, A search for interplanetary energetic particle events from solar posteruptive arcades. Astrophys. J. 533(2), 1063-1070 (2000). doi:10.1086/308680

Y.Y. Kartavykh, W. Dröge, B. Klecker, L. Kocharov, G.A. Kovaltsov, E. Möbius, Charge state formation of energetic ultraheavy ions in a hot plasma. Astrophys. J. 681(2), 1653-1659 (2008). doi:10.1086/588649

Kasper et al., Space Sci. Rev. (2014, this issue)

B. Klecker, E. Möbius, M.A. Popecki, L.M. Kistler, H. Kucharek, M. Hilchenbach, Observation of energydependent ionic charge states in impulsive solar energetic particle events. Adv. Space Res. 38(3), 493497 (2006). doi:10.1016/j.asr.2005.04.042

S.M. Krimigis, D.G. Mitchell, D.C. Hamilton, S.A. Livi, J. Dandouras, S. Jaskulek, T.P. Armstrong, J.D. Boldt, A.F. Cheng, G. Gloeckler, J.R. Hayes, K.C. Hsieh, W.-H. Ip, E.P. Keath, E. Kirsch, N. Krupp, L.J. Lanzerotti, R.A. Lundgren, B.H. Mauk, R.W. McEntire, E.C. Roelof, C.E. Schlemm, B.E. Tossman, B. Wilken, D.J. Williams, Magnetospheric imaging instrument (MIMI) on the Cassini mission to Saturn/Titan. Space Sci. Rev. 114(1-4), 233-329 (2004). doi:10.1007/s11214-004-1410-8

D. Lario, R.B. Decker, Estimation of solar energetic proton mission-integrated fluences and peak intensities for missions traveling close to the Sun. Space Weather 9, S11003 (2011). doi:10.1029/2011SW000708

D. Lario, M.-B. Kallenrode, E.C. Roelof, S.M. Krimigis, A. Aran, B. Sanahuja, Radial and longitudinal dependence of solar 4-13 MeV and 27-37 MeV proton peak intensities and fluences: Helios and IMP-8 intensities. Astrophys. J. 653(2), 1531-1544 (2006). doi:10.1086/508982

M.A. Lee, Coupled hydromagnetic wave excitation and ion acceleration at an evolving Coronal/Interplanetary shock. Astrophys. J. Suppl. Ser. 158(1), 38-67 (2005). doi:10.1086/428753

J.A. le Roux, G.P. Zank, W.H. Matthaeus, Pickup ion acceleration by turbulent field-aligned electric fields in the slow low-latitude solar wind. J. Geophys. Res.., Space Phys. 107(A7), SSH 9-1 (2002). doi:10.1029/ 2001JA000285. CiteID 1138

R.A. Leske, R.A. Mewaldt, C.M.S. Cohen, A.C. Cummings, E.C. Stone, M.E. Wiedenbeck, T.T. von Rosenvinge, Solar isotopic composition as determined using solar energetic particles. Space Sci. Rev. 130(104), 195-205 (2007). doi:10.1007/s11214-007-9185-3

G. Li, G.P. Zank, O. Verkhoglyadova, R.A. Mewaldt, C.M.S. Cohen, G.M. Mason, M.I. Desai, Shock geometry and spectral breaks in large SEP events. Astrophys. J. 702(2), 998-1004 (2009). doi:10.1088/0004$637 \mathrm{X} / 702 / 2 / 998$

S. Liu, V. Petrosian, G.M. Mason, Stochastic acceleration of stochastic acceleration of ${ }^{3} \mathrm{He}$ and ${ }^{4} \mathrm{He}$ by parallel propagation plasma waves. Astrophys. J. 613(1), L81-L84 (2004). doi:10.1086/425070

G. Livadiotis, D.J. McComas, Beyond kappa distributions: exploiting Tsallis statistical mechanics in space plasmas. J. Geophys. Res. 114(A11), A11105 (2009). doi:10.1029/2009JA014352

G. Lutz, Semiconductor Radiation Detectors (Springer, Munich, 1999)

G.M. Mason, ${ }^{3}$ He-rich solar energetic particle events. Space Sci. Rev. 130(1-4), 231-242 (2007) 
G.M. Mason, M.I. Desai, J.E. Mazur, J.R. Dwyer, Energetic particles accelerated by shocks in the heliosphere: what is the source material? in The Physics of Collisionless Shocks: 4th Annual IGPP International Astrophysics Conference. AIP Conference Proceedings, vol. 781 (2005), pp. 219-226. doi: 10.1063/1.2032700

G.M. Mason, G. Gloeckler, Power law distributions of suprathermal ions in the quiet solar wind. Space Sci. Rev. 172(1-4), 241-251 (2012)

G.M. Mason, D.K. Haggerty, E.C. Roelof, M.I. Desai, C.M. Cohen, R.A. Mewaldt, J.R. Dwyer, Injection and interplanetary propagation of solar energetic particles observed on ACE. Am. Geophys. Union 43, SH43B-01 (2006)

G.M. Mason, G. Li, C.M.S. Cohen, M.I. Desai, D.K. Haggerty, R.A. Leske, R.A. Mewaldt, G.P. Zank, Interplanetary propagation of solar energetic particle heavy ions observed at $1 \mathrm{AU}$ and the role of energy scaling. Astrophys. J. 761(2), 104 (2012). doi:10.1088/0004-637X/761/2/104. 27 pp.

G.M. Mason, J.E. Mazur, J.R. Dwyer, ${ }^{3} \mathrm{He}$ enhancements in large solar energetic particle events. Astrophys. J. 525(2), L133-L136 (1999). doi:10.1086/312349

G.M. Mason, J.E. Mazur, J.R. Dwyer, J.R. Jokipii, R.E. Gold, S.M. Krimigis, Abundances of heavy and ultraheavy ions in ${ }^{3}$ He-rich solar flares. Astrophys. J. 606(1), 555-564 (2004). doi:10.1086/382864

G.M. Mason, C.K. Ng, B. Klecker, G. Green, Impulsive acceleration and scatter-free transport of about $1 \mathrm{MeV}$ per nucleon ions in (He-3)-rich solar particle events. Astrophys. J. 339, 529-544 (1989). doi:10.1086/ 167315

G.M. Mason, M.E. Wiedenbeck, J.A. Miller, J.E. Mazur, E.R. Christian, C.M.S. Cohen, A.C. Cummings, J.R. Dwyer, R.E. Gold, S.M. Krimigis, R.A. Leske, R.A. Mewaldt, P.L. Slocum, E.C. Stone, T.T. von Rosenvinge, Spectral properties of He and heavy ions in 3He-rich solar flares. Astrophys. J. 574(2), 1039-1058 (2002). doi:10.1086/341112

W.H. Matthaeus, G. Qin, J.W. Bieber, G.P. Zank, Nonlinear collisionless perpendicular diffusion of charged particles. Astrophys. J. 590(1), L53-L56 (2003). doi:10.1086/376613

B.H. Mauk, J.B. Blake, D.N. Baker, J.H. Clemmons, G.D. Reeves, H.E. Spence, S.E. Jaskulek, C.E. Schlemm, L.E. Brown, S.A. Cooper, J. Craft, J.F. Fennell, R.S. Gurnee, C.M. Hammock, J.R. Hayes, P.A. Hill, G.C. Ho, J.C. Hutcheson, A.D. Jacques, S. Kerem, D.G. Mitchell, K.S. Nelson, N. Paschalidis, E. Rossano, M.R. Stokes, J.H. Westlake, The energetic particle detector (EPD) investigation and the energetic ion spectrometer (EIS) for the magnetospheric multiscale (MMS) mission. Space Sci. Rev. (2014). doi:10.1007/s11214-014-0055-5

B.H. Mauk, D.K. Haggerty, S.E. Jaskulek, C.E. Schlemm, L.E. Brown, S.A. Cooper, R.S. Gurnee, C.M. Hammock, J.R. Hayes, G.C. Ho, J.C. Hutcheson, A.D. Jacques, S. Kerem, C.K. Kim, D.G. Mitchell, K.S. Nelson, C.P. Paranicas, N. Paschalidis, E. Rossano, M.R. Stokes, The Jupiter energetic particle detector instrument (JEDI) investigation for the Juno mission. Space Sci. Rev. (2013). doi:10.1007/ s11214-013-0025-3 (online first)

J.E. Mazur, G.M. Mason, J.R. Dwyer, J. Giacalone, J.R. Jokipii, E.C. Stone, Interplanetary magnetic field line mixing deduced from impulsive solar flare particles. Astrophys. J. 532(1), L79-L82 (2000)

D.J. McComas, M. Velli, W.S. Lewis, L.W. Acton, M. Balat-Pichelin, V. Bothmer, R.B. Dirling Jr., W.C. Feldman, G. Gloeckler, S.R. Habbal, D.M. Hassler, I. Mann, W.H. Matthaeus, R.L. McNutt Jr., R.A. Mewaldt, N. Murphy, L. Ofman, E.C. Sittler Jr., C.W. Smith, T.H. Zurbuchen, Understanding coronal heating and solar wind acceleration: case for in situ near-sun measurements. Rev. Geophys. 45(1), RG1004 (2007). doi:10.1029/2006RG000195

R.L. McNutt, S.A. Livi, R.S. Gurnee, M.E. Hill, K.A. Cooper, G.B. Andrews, E.P. Keath, S.M. Krimigis, D.G. Mitchell, B. Tossman, F. Bagenal, J.D. Boldt, W. Bradley, W.S. Devereux, G.C. Ho, S.E. Jaskulek, T.W. LeFevere, H. Malcom, G.A. Marcus, J.R. Hayes, G.T. Moore, M.E. Perry, B.D. Williams, P. Wilson, L.E. Brown, M.B. Kusterer, J.D. Vandegriff, The Pluto Energetic Particle Spectrometer Science Investigation (PEPSSI) on the new horizons mission. Space Sci. Rev. 140(1-4), 315-385 (2008)

R.A. Mewaldt, E.C. Stone, R.E. Vogt, Neutral particle background in cosmic Ray telescopes composed of silicon solid state detectors. Space Sci. Instrum. 3, 231-242 (1977)

R.A. Mewaldt, C.M.S. Cohen, G.M. Mason, A.W. Labrador, M.L. Looper, D.E. Haggerty, C.G. Maclennan, A.C. Cummings, M.I. Desai, R.A. Leske, G. Li, J.E. Mazur, E.C. Stone, M. Wiedenbeck, Solar energetic particle spectral breaks, in The Physics of Collisionless Shocks: 4th Annual IGPP International Astrophysics Conference. AIP Conference Proceedings, vol. 781 (2005), pp. 227-232. doi:10.1063/ 1.2032701

R.A. Mewaldt, Solar energetic particle composition, energy spectra, and space weather. Space Sci. Rev. 124(1-4), 303-316 (2006). doi:10.1007/s11214-006-9091-0

R.A. Mewaldt, C.M.S. Cohen, J. Giacalone, G.M. Mason, E.E. Chollet, M.I. Desai, D.K. Haggerty, M.D. Looper, R.S. Selesnick, A. Vourlidas, How efficient are coronal mass ejections at accelerating solar energetic particles? in Particle Acceleration and Transport in the Heliosphere and Beyond: 7th Annual 
International Astrophysics Conference. AIP Conference Proceedings, vol. 1039 (2008a), pp. 111-117. doi:10.1063/1.2982431

R.A. Mewaldt, C.M.S. Cohen, W.R. Cook, A.C. Cummings, A.J. Davis, S. Geier, B. Kecman, J. Klemic, A.W. Labrador, R.A. Leske, H. Miyasaka, V. Nguyen, R.C. Ogliore, E.C. Stone, R.G. Radocinski, M.E. Wiedenbeck, J. Hawk, S. Shuman, T.T. von Rosenvinge, K. Wortman, The low-energy telescope (LET) and SEP central electronics for the STEREO mission. Space Sci. Rev. 136(1-4), 285-362 (2008b)

R.A. Mewaldt, R.A. Leske, E.C. Stone, A.F. Barghouty, A.W. Labrador, C.M.S. Cohen, A.C. Cummings, A.J. Davis, T.T. von Rosenvinge, M.E. Wiedenbeck, STEREO observations of energetic neutral hydrogen atoms during the 2006 December 5 solar flare. Astrophys. J. 693(1), L11-L15 (2009). doi:10.1088/0004-637X/693/1/L11

R.A. Mewaldt, M.D. Looper, C.M.S. Cohen, D.K. Haggerty, A.W. Labrador, R.A. Leske, G.M. Mason, J.E. Mazur, T.T. von Rosenvinge, Energy spectra, composition, and other properties of ground-level events during solar cycle 23. Space Sci. Rev. 171(1-4), 97-120 (2012a). doi:10.1007/s11214-012-9884-2

R.A. Mewaldt, G.M. Mason, C.M.S. Cohen, The dependence of solar energetic particle fluences on suprathermal seed-particle densities, in Space Weather: The Space Radiation Environment. AIP Conference Proceedings, vol. 1500 (American Institute of Physics, Melville, 2012b), pp. 128-133

D.G. Mitchell, L.J. Lanzerotti, C.K. Kim, M. Stokes, G. Ho, S. Cooper, A. Ukhorskiy, J.W. Manweiler, S. Jaskulek, D.K. Haggerty, P. Brandt, M. Sitnov, K. Keika, J.R. Hayes, L.E. Brown, R.S. Gurnee, J.C. Hutcheson, K.S. Nelson, N. Paschalidis, E. Rossano, S. Kerem, Radiation belt storm probes ion composition experiment (RBSPICE). Space Sci. Rev. 179(1-4), 263-308 (2013). doi:10.1007/s11214-013-9965-x

NASA Science Mission Directorate, Solar probe plus announcement of opportunity, Solicitation: NNH10ZDA002O. http://nspires.nasaprs.com/external/solicitations/summary.do?method=init\&solId= \{BC6CAAB3-F512-B60F-5F11-2942EAACFA8B $\} \&$ path=closedPast. Accessed 4 June 2014

C.K. Ng, D.V. Reames, A.J. Tylka, Modeling shock-accelerated solar energetic particles coupled to interplanetary Alfvén waves. Astrophys. J. 591(1), 461-485 (2003). doi:10.1086/375293

N.P. Paschalidis, The power energy ASIC, with voltage and time-over-threshold read outs: application to space science particle instruments, in IEEE Nuclear Science Symposium and Medical Imaging Conference, N02-5, Dresden, Germany (2008)

V. Petrosian, Y.W. Jiang, S. Liu, G.C. Ho, G.M. Mason, Relative distributions of fluences of $3 \mathrm{He}$ and $4 \mathrm{He}$ in solar energetic particles. Astrophys. J. 701(1), 1-7 (2009)

M. Pick, G.M. Mason, Y.-M. Wang, C. Tan, L. Wang, Solar source regions for 3He-rich solar energetic particle events identified using imaging radio, optical, and energetic particle observations. Astrophys. J. 648(2), 1247-1255 (2006). doi:10.1086/505926

A.F. Rappazzo, W.H. Matthaeus, D. Ruffolo, S. Servidio, M. Velli, Interchange reconnection in a turbulent corona. Astrophys. J. Lett. 758(1), L14 (2012). doi:10.1088/2041-8205/758/1/L14, 6 pp.

D.V. Reames, Particle acceleration at the Sun and in the heliosphere. Space Sci. Rev. 90(3/4), 413-491 (1999). doi:10.1023/A:1005105831781

D.V. Reames, The two sources of solar energetic particles. Space Sci. Rev. 175(1-4), 53-92 (2013). doi:10. 1007/s11214-013-9958-9

D.V. Reames, C.K. Ng, Streaming-limited intensities of solar energetic particles. Astrophys. J. 504, 1002 (1998). doi:10.1086/306124

W.K.M. Rice, G.P. Zank, G. Li, Particle acceleration and coronal mass ejection driven shocks: shocks of arbitrary strength. J. Geophys. Res. 108(A10), SSH 5-1 (2003). doi:10.1029/2002JA009756. CiteID 1369

D. Ruffolo, W.H. Matthaeus, P. Chuychai, Trapping of solar energetic particles by the small scale topology of solar wind turbulence. Astrophys. J. 597(2), L169-L172 (2003). doi:10.1086/379847

N.A. Schwadron, M.A. Dayeh, M.I. Desai, H. Fahr, J.R. Jokipii, M.A. Lee, Superposition of stochastic processes and the resulting particle distributions. Astrophys. J. 713(2), 1386-1392 (2010). doi:10.1088/ 0004-637X/713/2/1386

A. Seripienlert, D. Ruffolo, W.H. Matthaeus, P. Chuychai, Dropouts in solar energetic particles: associated with local trapping boundaries or current sheets? Astrophys. J. 711(2), 980-989 (2010). doi:10.1088/ 0004-637X/711/2/980

B. Teaca, M.S. Wield, F. Junco, R. Schlickeiser, Acceleration of particles in imbalanced magnetohydrodynamic turbulence (March 2014). arXiv:1403.3000

J. Tessein, W.H. Matthaeus, M. Wan, K.T. Osman, D. Ruffolo, J. Giacalone, Association of suprathermal particles with coherent structures and shocks. Astrophys. J. Lett. 776(1), L8 (2013). doi:10.1088/20418205/776/1/L8, 4 pp.

A.J. Tylka, M.A. Lee, A model for spectral and compositional variability at high energies in large, gradual solar particle events. Astrophys. J. 646(2), 1319-1334 (2006). doi:10.1086/505106 
A.J. Tylka, D.V. Reames, C.K. Ng, Observations of systematic temporal evolution in elemental composition during gradual solar energetic particle events. Geophys. Res. Lett. 26(14), 2141-2144 (1999). doi:10.1029/1999GL900458

P. van Nes, R. Reinhard, T.R. Sanderson, K.-P. Wenzel, R.D. Zwickl, The energy spectrum of 35- to 1600-keV protons associated with interplanetary shocks. J. Geophys. Res. 89, 2122-2132 (1984). doi:10.1029/ JA089iA04p02122

T.T. von Rosenvinge, D.V. Reames, R. Baker et al., The high energy telescope for STEREO. Space Sci. Rev. 136(1-4), 391-435 (2008)

L. Wang, Personal communication (2010)

Y.-M. Wang, M. Pick, G.M. Mason, Coronal holes, jets, and the origin of 3He-rich particle events. Astrophys. J. 639(1), 495-509 (2006). doi:10.1086/499355

G. Wibberenz, H.V. Cane, Multi-spacecraft observations of solar flare particles in the inner heliosphere. Astrophys. J. 650(2), 1199-1207 (2006). doi:10.1086/506598

M.E. Wiedenbeck, C.M.S. Cohen, A.C. Cummings et al., Persistent energetic 3 He in the inner heliosphere, in Proceedings of the 30th International Cosmic Ray Conference, July 3-11, vol. 1 (2008), pp. 91-94 